\title{
Computer Assisted Interpretation of the Human EEG
}

Improving diagnostic efficiency and consistency in clinical reviews

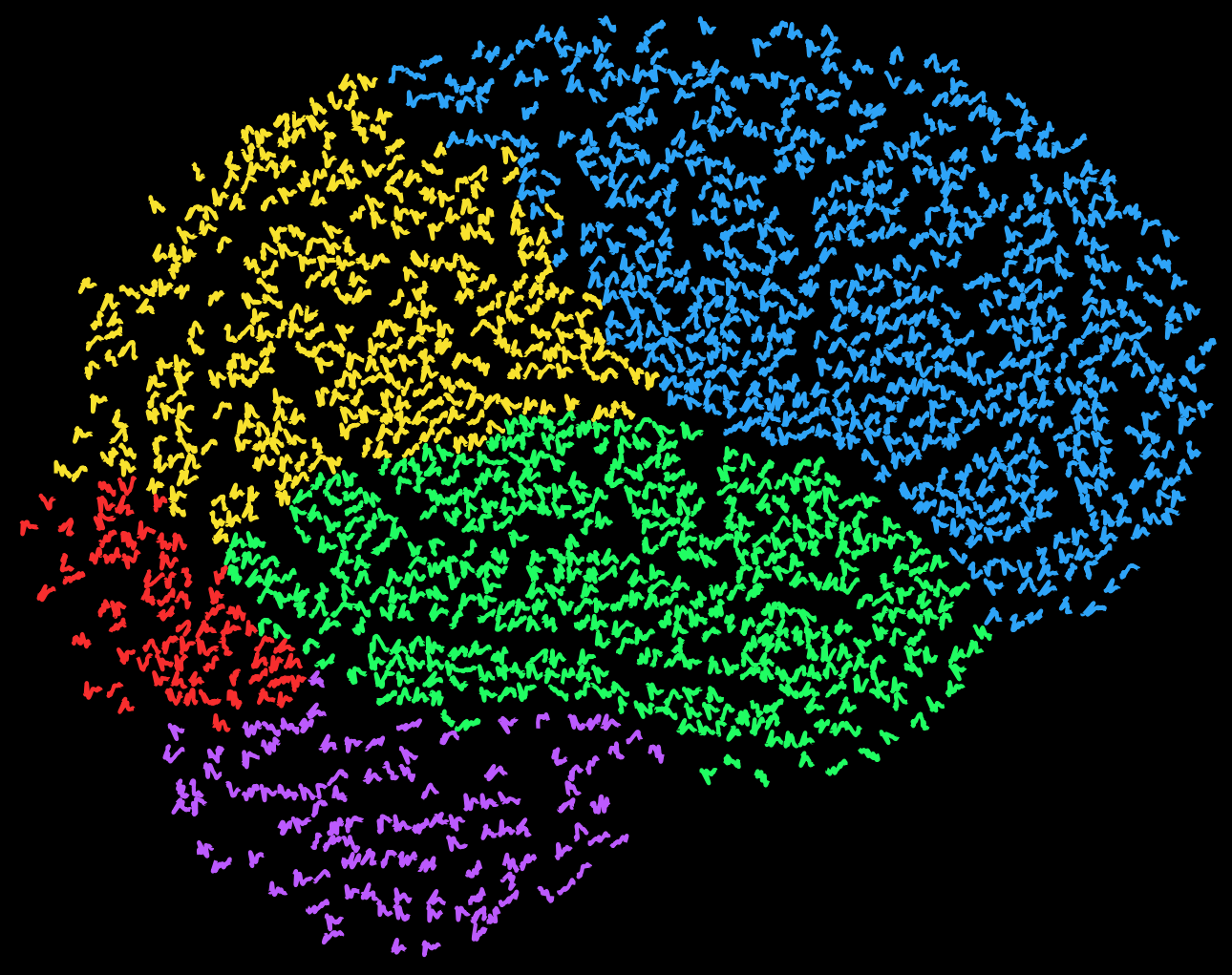

Shaun S. Lodder

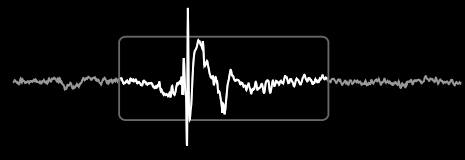




\title{
COMPUTER ASSISTED INTERPRETATION OF THE HUMAN EEG
}

\author{
IMPROVING DIAGNOSTIC EFFICIENCY AND \\ CONSISTENCY IN CLINICAL REVIEWS
}

SHAUN S. LODDER 


\section{Chairman:}

Prof. dr. Gerard van der Steenhoven University of Twente

\section{Promotor:}

Prof. dr. ir. Michel J.A.M. van Putten University of Twente

\section{Committee members:}

Dr. Harald Aurlien

Prof. dr. Fernando H. Lopes da Silva

Prof. dr. Cornelis J. Stam

Dr. Frans S.S. Leijten

Prof. dr. Stephan A. van Gils

Prof. dr. ir. Raymond N.J. Veldhuis
Haukeland University Hospital, Norway University of Amsterdam

VU University Medical Center, Amsterdam

University Medical Center, Utrecht

University of Twente

University of Twente

Computer Assisted Interpretation of the Human EEG

Shaun S. Lodder

Printed by Gildeprint, Enschede, The Netherlands

ISBN: 978-90-365-3592-2

DOI: $\quad 10.3990 . / 1.9789036535922$

Cover design by Shaun Lodder. The picture depicts a brain drawn with 1485 templates of inter-ictal epileptiform discharges extracted from routine scalp EEG recordings.

() 2013 Shaun S. Lodder 


\title{
COMPUTER ASSISTED INTERPRETATION OF THE HUMAN EEG
}

\author{
IMPROVING DIAGNOSTIC EFFICIENCY AND \\ CONSISTENCY IN CLINICAL REVIEWS
}

\section{DISSERTATION}

to obtain

the degree of doctor at the University of Twente, on the authority of the rector magnificus,

Prof. dr. H. Brinksma,

on account of the decision of the graduation committee, to be publicly defended on

Friday, $31^{\text {st }}$ January 2014 , at $14: 45$

by

Shaun Sandy Lodder

born on October 20 $0^{\text {th }} 1984$

in Kimberley, South Africa 
This thesis has been approved by:

\section{Promotor}

Prof. dr. ir. Michel J.A.M. van Putten 
To My Parents

Hugo and Leonie Lodder

Try. Make mistake. Fail. Learn.

Try better. Make mistake. Fail. Learn.

Try better still. Make mistake. Fail. Learn.

Repeat until...

Try. Succeed.

(Ken Evoy) 



\section{CONTENTS}

Chapter 1 Introduction 1

$\begin{array}{lll}\text { Part I } & \text { EEG background pattern } & 15\end{array}$

Chapter 2 Estimation of the Posterior Dominant Rhythm 17

Chapter 3 Quantification of the adult EEG background pattern 35

Chapter $4 \quad$ Clinical evaluation of computer-assisted interpretation $\quad 59$

$\begin{array}{lll}\text { Part II Inter-ictal epileptiform discharges } & 75\end{array}$

Chapter 5 Inter-ictal spike detection using smart templates $\quad 77$

Chapter 6 A self-adapting system for finding inter-ictal epileptiform discharges 95

$\begin{array}{lll}\text { Chapter } 7 & \text { Summary and general discussion } & 113\end{array}$

$\begin{array}{lll}\text { Chapter } 8 \quad \text { Nederlandse samenvatting } & 127\end{array}$

$\begin{array}{ll}\text { References } & 133\end{array}$

$\begin{array}{ll}\text { Acknowledgments } & 151\end{array}$

$\begin{array}{ll}\text { About the author } & 155\end{array}$ 

Chapter 1

Introduction 
Scalp electroencephalography (EEG) is one of the most commonly used techniques for non-invasive measurements of cortical brain activity. With the first recording made in humans close to a century ago, this technology is far from new (Berger, 1929). Even so, interpretation of the signals has remained too complex to completely automate, and experienced clinicians are still required to analyze the data through a tedious process of visual inspection (Ebersole and Pedley, 2003; Niedermeyer and da Silva, 2004). In contrast to other modern day technologies such as $\mathrm{fMRI}$ and PET which make extensive use of computational methods to visualize the recorded brain activity, EEG is mostly still reviewed in its raw form, i.e. multiple lines of time series each representing the electrical activity measured by individual electrodes on the scalp, as illustrated in Fig. 1.1.

EEG measures tiny voltage fluctuations of electrical activity on the scalp resulting from ionic currents produced by the firing of neurons in the brain (Niedermeyer and da Silva, 2004). Due to low voltages and interference from muscle activity and other artifacts (EEG voltages are typically within the $50 \mu \mathrm{V}$ range where artifacts can be one or two orders of magnitude higher), the signals have a low signal to noise ratio and the captured neuronal activity is mostly limited to the cortical layers close to the scalp. The result of volume conduction from bone and cerebrospinal fluid also causes a "smearing" effect across neighboring electrode channels, yielding a lower spatial resolution than some other non-invasive techniques (Ebersole and Pedley, 2003). However, in contrast to other non-invasive methods, EEG has a high temporal resolution and provides a portable and cost-effective technology for many diagnostic procedures. Given the general availability of EEG equipment and its flexibility in recording environments, it will continue to play an important role in clinical procedures ranging from epilepsy diagnostics and ICU monitoring to rehabilitative technologies and in-home patient monitoring (Wilson and Emerson, 2002; Thakor and Tong, 2004; Friedman and Hirsch, 2009; Kurtz et al., 2009; Young, 2009; Casson and Rodriguez-Villegas, 2009; Arciniegas, 2011; Faulkner et al., 2012; Beniczky et al., 2013; Sanchez et al., 2013; Halford et al., 2010).

\section{Common EEG properties}

The common properties observed in EEG recordings can be divided into two main categories: (i) background activity, and (ii) transients (Ebersole and Pedley, 2003; Niedermeyer and da Silva, 2004). During a routine EEG review, the properties in these two categories are carefully evaluated in order to search for abnormalities or 


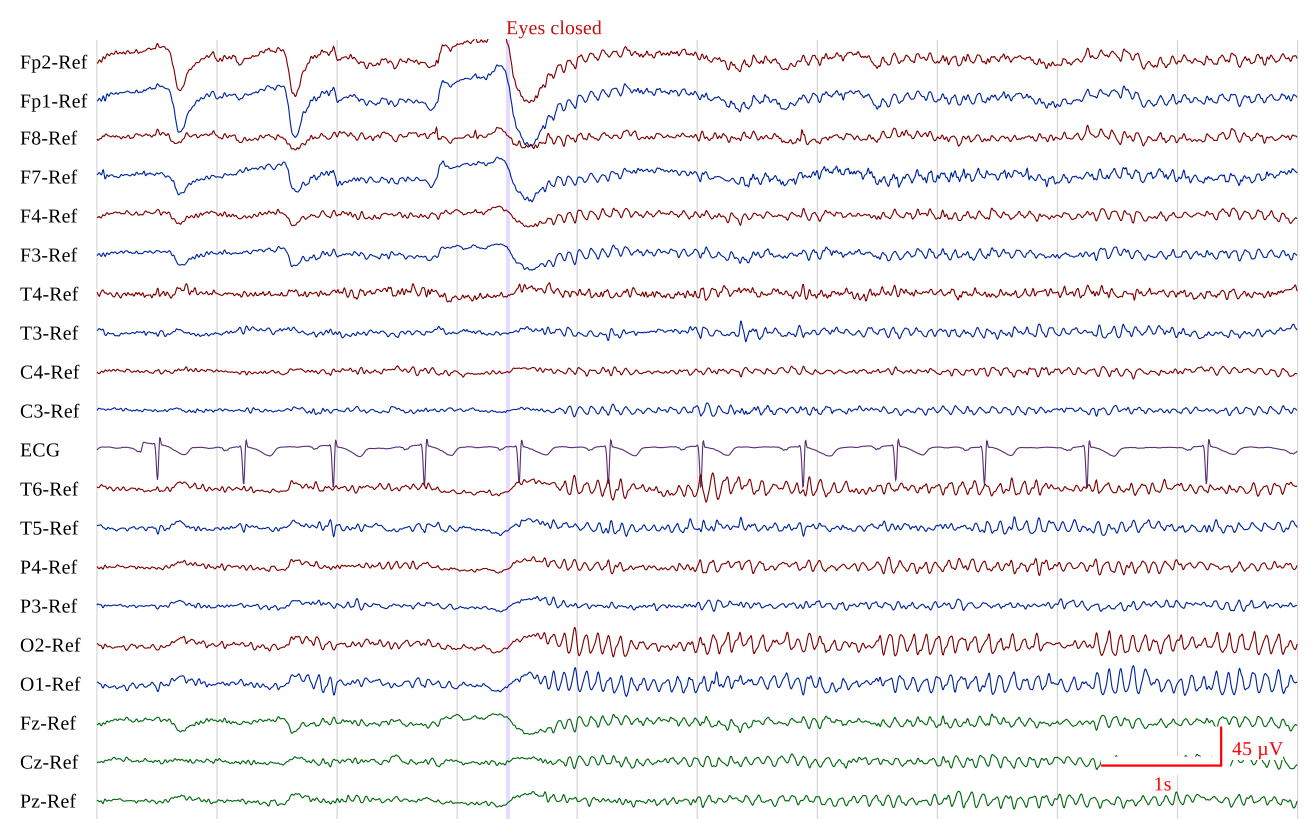

Fig. 1.1: A 19-channel scalp EEG recording in common reference montage showing a reactive posterior dominant rhythm, most clearly seen over the occipital region (channels $\mathrm{O} 1$ and O2) after the eyes are closed at $3.5 \mathrm{~s}$.

deviations from the norm. Transient activity in the form of inter-ictal epileptiform discharges are especially relevant for the diagnosis of epilepsy, and most routine EEGs are typically made based on this as diagnostic purpose. Below is a brief outline of the most common EEG properties.

\section{Background activity}

Background activity describes the mean properties observed on a global scale in the EEG (Ebersole and Pedley, 2003; Niedermeyer and da Silva, 2004). Most of these properties relate to rhythmic behavior as observed on the scalp, and these rhythms are typically described within the frequency bands that they occur: delta $(<4 \mathrm{~Hz})$, theta $(4-8 \mathrm{~Hz})$, alpha $(8-12 \mathrm{~Hz})$, beta $(12-30 \mathrm{~Hz})$ and gamma $(30-100+\mathrm{Hz})($ Niedermeyer and da Silva, 2004). Well known properties exist for each type, and abnormalities or deviations most often point to disease or brain damage.

A very well known part of the EEG background activity, and also the first rhythm to be discovered, is the alpha rhythm (Berger, 1929). Its peak frequency is an important marker to monitor maturation of the human brain in early life (Niedermeyer 
and da Silva, 2004; Aurlien et al., 2004), and slowing in adults is coupled with various neurodegenerative diseases (Jeong, 2004; Dauwels et al., 2010). The alpha rhythm, also referred to as the posterior dominant rhythm, is best seen over the occipital region in a healthy subject. It gradually decreases in amplitude as it moves towards the anterior, and has a peak frequency related to the subject's age (Niedermeyer and da Silva, 2004). The alpha rhythm is most clearly observed during a relaxed state of wakefulness, typically when the eyes are closed, and it is reactive to external stimuli such as auditory or visual input.

Other well known background rhythms are the mu $(8-13 \mathrm{~Hz})$ and beta rhythms (Ebersole and Pedley, 2003). The mu rhythm is most prominent over the motor cortex and responds to motor execution and imagery. Beta rhythms are mostly observed during an active awake state and are related to cognitive and motor execution tasks. Lastly, considering slower activity, children display more delta and theta activity, whereas in adults a significant presence of slow activity is considered to be abnormal.

Apart from the frequency and amplitude of the oscillations themselves, the background activity also has a number of other relevant properties (Beniczky et al., 2013). A healthy brain shows a high degree of symmetry between left and right hemispheres. Symmetry can be seen in both the frequency and amplitude, and asymmetries usually point to focal abnormalities associated with many pathological conditions, ranging from ischaemia and trauma to space occupying lesions (Ebersole and Pedley, 2003; Niedermeyer and da Silva, 2004). In addition to symmetry, the EEG also shows an anterior-to-posterior gradient in both frequency and amplitude. Rhythms with higher frequencies and lower amplitudes are most dominant over the anterior, whereas slower oscillations with higher amplitudes are commonly observed over posterior regions. A lack of fast activity or no anterior-posterior gradient, especially in adults, is considered abnormal and can for example be observed in coma and neurodegenerative diseases (Ebersole and Pedley, 2003).

\section{Transients}

Transients refer to short, abrupt changes in the EEG and can be caused by both normal and abnormal processes in the brain (Ebersole and Pedley, 2003; Niedermeyer and da Silva, 2004). Examples of normal transients are sleep spindles, Kcomplexes, lambda waves and wicket waves (Niedermeyer and da Silva, 2004). The most common form of abnormal transients are inter-ictal epileptiform discharges 
(IEDs). These appear in the form of spikes, sharp waves, poly-spikes and spike and slow-wave discharges (Niedermeyer and da Silva, 2004). An example of spike and slow-wave discharges is shown in Fig. 1.2 with the IEDs marked in gray. In EEG, the presence of IEDs are correlated with a high likelihood of recurrent seizures. They are almost exclusively present in epileptic patients, and their presence play an important role in the diagnosis and classification of epilepsy, in particular when seizures themselves cannot be observed. Hyperventilation is the most effective method for activating ictal and inter-ictal epileptiform activity in children with absence epilepsy. For provoking generalized seizures or inter-ictal discharges related to reflex epilepsy, external stimuli such as photic stimulation works best (Ebersole and Pedley, 2003). In addition to performing hyperventilation and photic stimulation during routine epilepsy screening, sleep deprivation can also be used to improve the chances of finding inter-ictal events. Sleep activates the occurrence of epileptiform discharges in about one third of epilepsy patients (Ebersole and Pedley, 2003).

\section{Artifacts}

Apart from background activity and transients, EEGs are also plagued by artifacts which do not originate from neuronal activity. This is also one of the main reasons why the automation of EEG analysis have remained so difficult until now (Anderson and Doolittle, 2010; Tatum et al., 2011a). Although more exist, common artifacts are: (i) myogenic artifacts caused by muscle movements, (ii) eye-blink artifacts, (iii) pulsation artifacts from electrodes placed on top of arteries, (iv) electrodermal artifacts causing slow DC shifts, $(v)$ power line noise $(50$ or $60 \mathrm{~Hz}$ depending on the region), and (vi) electrical or mechanical noise from nearby instrumentation (Klass, 1995; Ille et al., 2002; Tatum et al., 2011b; Niedermeyer and da Silva, 2004). In Fig. 1.2 an example of a muscle artifact can be seen in approximately the middle of the epoch, mostly over the frontal and temporal regions.

Many artifact detection and removal techniques have been suggested, all with their own strengths and weaknesses (van de Velde et al., 1999; Vigário et al., 2000; Castellanos and Makarov, 2006; Delorme et al., 2007). Popular solutions are spatial filtering and independent component analysis (ICA) based filters. Spatial filters attempt to separate signal sources generated from the cerebral cortex from other contributions unrelated to neuronal activity (Ille et al., 2002). ICA filters use the concept of blind source separation to obtain individual sources of activity (Vigário et al., 


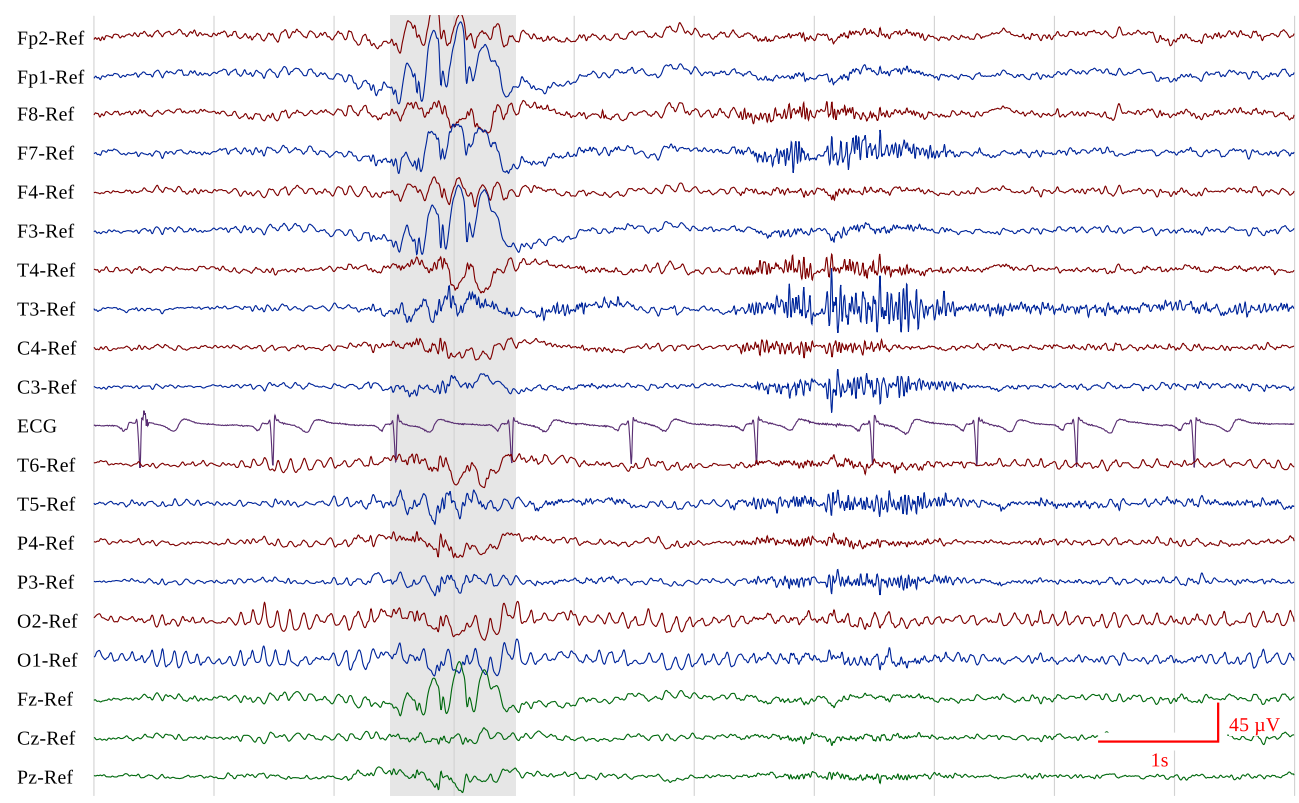

Fig. 1.2: An example of four consecutive spike and slow-wave discharges (marked in gray) that form part of inter-ictal epileptiform activity (IEDs). The presence of IEDs in an EEG are correlated with a high likelihood of recurrent seizures and their presence therefore plays an important role in the diagnosis of epilepsy. A muscle artifact is also seen at $6 \mathrm{~s}$. Artifacts corrupt the signal and can make it difficult to measure the underlying neuronal activity.

2000). After the individual sources have been obtained, artifact sources are identified and removed, and the remaining sources are mapped back to their original space. Regardless of the technique used to remove artifacts, human intervention is still required to confirm that no important information will be lost during filtering. This intermediate step prevents full automation, but the (reasonably) artifact-free EEG allows quantitative algorithms to achieve higher accuracies.

\section{Visual inspection and conventional reviews}

Given the complexity and variability in EEG recordings, conventional reviews consist of analyzing the EEG in its raw form, as shown in Fig. 1.1 and 1.2. Using a number of different referencing montages (eg. bi-polar, source, common-reference), the reviewer looks for abnormalities or deviations in the EEG properties described above. Depending on the clinical question at hand, the reviewer can then create a report and make an interpretation based on his findings from the review (Ebersole 
and Pedley, 2003; Niedermeyer and da Silva, 2004). Unfortunately, this process is slow and requires an expert to perform. EEGs are typically viewed iteratively over short epochs of ten to thirty seconds at a time. For long recordings this process becomes very tedious and lowers a reviewers concentration. Given this visual burden and the costs involved in reviewing, routine EEG recordings are typically limited to 20 or $30 \mathrm{~min}$ in length. For epilepsy however, it has been shown that a longer recording window can improve the chances of finding inter-ictal epileptiform activity. In many cases, a single long-term ambulatory recording can therefore avoid the need for patients to return for follow-up recordings (Doppelbauer et al., 1993; Faulkner et al., 2012; Halford et al., 2010).

Although automated analysis is not yet accurate or diverse enough to replace visual reviews, a computer's ability to perform complex calculations can be of great benefit to the reviewer. By having an automated method search for areas of interest in the EEG and then presenting only these findings to the reviewer, the visual burden can be minimized and review times can be reduced significantly (Scherg et al., 2012; Anderson and Doolittle, 2010). In addition, consistent feedback from the automated methods and more rigid definitions can improve inter-rater reliability and allow for more consistent reports (Halford et al., 2011). Computer-assisted EEG interpretation also allows reviewers to easily extract and visualize certain properties in the EEG, as shown in (van Putten, 2008; van Putten et al., 2004; Friedman and Hirsch, 2010). With faster review times, better visualization of the underlying neuronal activity, and still the same diagnostic certainty and reliability as compared to visual inspection by itself, the addition of automated analysis (as shown in Fig. 1.3) brings many possibilities to life.

\section{Computer-assisted EEG interpretation}

Computerized analysis, better known as quantitative EEG (QEEG), has been around for some time (Martin et al., 1972; Nakamura et al., 1992; Jordan, 1995; Thakor and Tong, 2004; Anderson and Wisneski, 2008). A vast number of automated detection algorithms have been proposed since the introduction of digital EEG, and although most studies only focus on describing one feature or property of the EEG, the wide range of its use have shown that QEEG features can be of great benefit during clinical diagnosis and monitoring. Clinical applications for QEEG include ICU monitoring (Friedman and Hirsch, 2010; Cloostermans et al., 2011; Foreman and Claassen, 2012), clinical psychiatry (Coburn et al., 2006; Hammond, 2010), and the diagnosis 


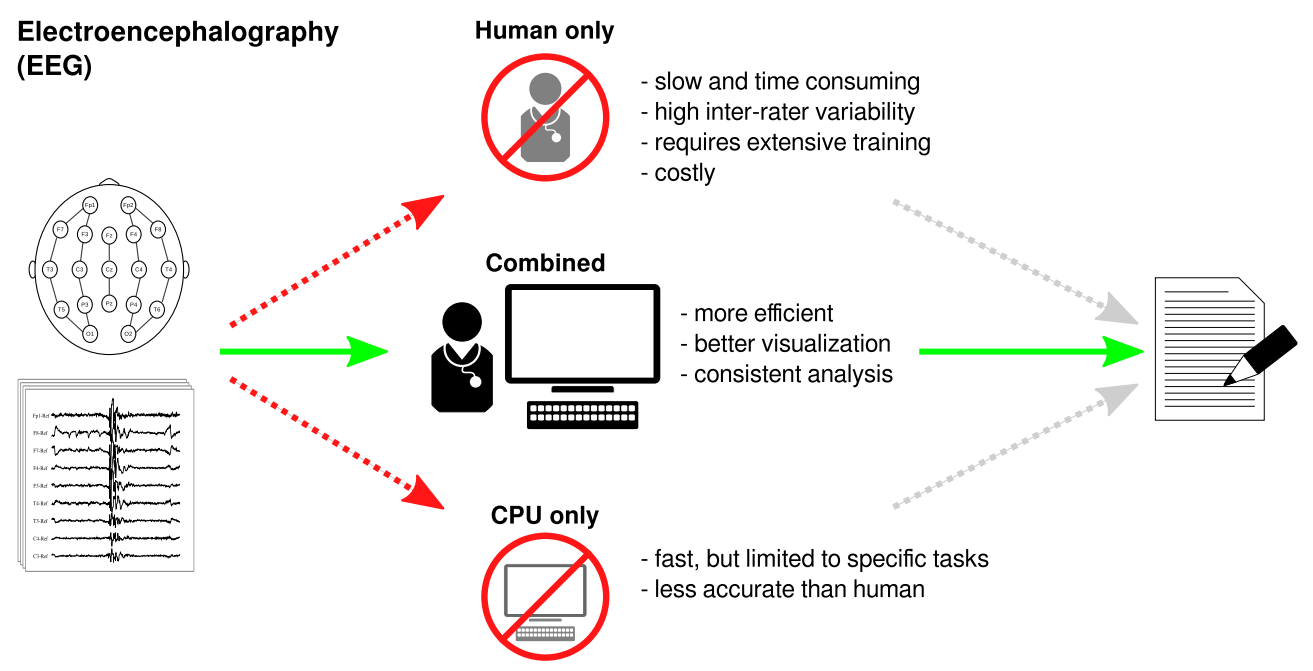

Fig. 1.3: Visual review of EEG is time-consuming, requires extensive training and suffers from high inter-rater variability. Computerized interpretation is consistent and fast, but is limited to specific tasks and lacks the accuracy of human interpretation. We aim to combine computerized interpretation with visual reviews to improve reviewer efficiency and lower inter-rater variability.

of neurodegenerative diseases (Petit et al., 2004; Babiloni et al., 2011; Moretti et al., 2012). Although a number of commercial applications exist, computer-assisted interpretation and structured reports are still not widely accepted by all (Thatcher, 2010). Extensive reviews on quantitative EEG and automated spike detection have been performed in the past, some more recent than others (Nuwer, 1997; Wilson and Emerson, 2002; Halford, 2009; Anderson and Doolittle, 2010). Although not a full review is given here, the two sections that follow provide an overview of relevant work leading up to the objectives and subsequent investigations described in this thesis.

\section{Background activity}

The main research focus in computer-assisted reviews for routine outpatient EEG recordings up to now has mostly been on the detection of inter-ictal epileptiform discharges (Nuwer, 1997; Wilson and Emerson, 2002; Halford, 2009). Although commercial applications are available to help clinicians visualize some of the common properties in $\mathrm{EEG}^{1}$, their focus also mainly lies in seizure and spike detection, intensive care monitoring and source localization. Regarding the clinical use of auto-

\footnotetext{
${ }^{1}$ For example applications see http://www.persyst.com/ and http://www.besa.de/.
} 
mated systems that can analyze and generate EEG reports compatible with those of reviewers on common background properties such as the alpha rhythm frequency and reactivity, anterior-posterior gradients, symmetry and slowing, clinical implementations could not be found.

The current best practice for reviewing EEGs involves visual analysis of the recording in its raw form followed by a written report based on the findings in free-text. The latter part can lead to a high degree of variability and inconsistency between reviewers (Haut et al., 2002; Benbadis et al., 2009; Gerber et al., 2008; Azuma et al., 2003), and given that the findings are not noted down using a set category of outcomes or that no commonly accepted guidelines exist for describing some properties, reports become difficult to query and compare to the findings of other clinicians. In recent work by Beniczky et al. (2013), the authors describe a set of guidelines and definitions (including the reporting of common background properties) that is being constructed as part of a pan-European project with the goal of providing more consistency and structure for the reports in clinical EEG reviews (Beniczky et al., 2013; Aurlien et al., 2004, 2007). Standard procedure for writing EEG reports state that objective observations of the EEG properties should be separate from the conclusions made by the reviewer based on these observations. ${ }^{2}$ As such, quantitative analysis is well suited for the objective description of the background properties, and in combination with the guidelines provided by (Beniczky et al., 2013), if widely accepted, a more structured and consistent report can be generated. This should lead to more consistency in reviews and make patient databases easier to query for patient information. Given that other factors such as medication and patient history are not known or taken into account by automated systems, the final conclusions in EEG reports should always be drawn by the reviewer.

Apart from improving consistency in reporting, there is also a need to find faster and more intuitive ways to visualize and interpret EEGs and thereby lessen the burden of visual analysis (Nuwer, 1997; Aurlien et al., 2004; van Putten, 2008; Halford, 2009; Anderson and Doolittle, 2010). A number of quantitative EEG features have been proposed to describe specific properties in the EEG. They include statistical measures such as variance, kurtosis and skewness (Scherg et al., 2012; Stevenson et al., 2013), non-linear energy operators (Mukhopadhyay and Ray, 1998), smallworld networks and functional connectivity (Stam et al., 2007; Bullmore and Sporns, 2009), synchrony (Lachaux et al., 1999; van Putten, 2003), entropy (Stam, 2005; Kan-

\footnotetext{
${ }^{2}$ See Guideline 7 provided by the American Clinical Neurophysiology Society, J Clin Neurophysiol. 2006 Apr;23(2):118-21.
} 
nathal et al., 2005), power ratios (Kurtz et al., 2009; Cloostermans et al., 2011), bispectral index (Sigl and Chamoun, 1994), and left-right symmetry (van Putten et al., 2004). Despite the variety of complex features available, relatively simple measures can be used to describe many of the common background properties of an EEG (van Putten, 2008). Example features are the presence or absence of certain rhythmic components, power ratios between delta-, theta-, alpha- and beta-bands, and the power distribution over the scalp. The importance of each background property will vary based on the reason for recording, but in general, a description of the background pattern is of significant importance to any review.

\section{Inter-ictal epileptiform spike detection}

As shown in two detailed reviews by Wilson and Emerson (2002) and Halford (2009), more than 50 inter-ictal epileptiform spike detection methods have been reported since the 1970's. Additional methods after these reviews include (Nonclercq et al., 2009, 2012; Ji et al., 2011a; Scherg et al., 2012). Promising results have been achieved if we look at the performance criteria chosen, and indeed, a substantial amount of research has been done in automated spike detection. Regardless of all the accomplishments however, very few commercially available systems exist that implement automated inter-ictal spike detection, and although some show more success than others, it can be argued that none of these have reached mainstream acceptance. This shows that although the problem of automated spike detection is almost as old as digital EEG itself (Gotman and Gloor, 1976), it has still not been solved, and given that longer recordings show improved chances of finding interictal events if any exist (Friedman and Hirsch, 2009; Faulkner et al., 2012), automated detection may be of even more importance today than it was before.

One of the main concerns in automated spike detection, as discussed by Halford (2009), is the fact that each publication uses its own EEG dataset, thereby making it difficult and inaccurate to compare the results between various detection methods. Unless the same dataset is used, one cannot fairly determine the sensitivity or false detection rate for at least the following two reasons. First, recordings may not be of the same length or contain the same number of IEDs. Given that it will be easier to detect many events in a short recording than a small number events in a long recording, the method's thresholds and parameters will be chosen in such a way as to optimize the its false detection rate. Secondly, apart from IEDs, systems such as these often mistakenly detect eye blinks and other artifacts as epileptiform 
discharges. If the number of artifacts vary greatly or their morphology matches inter-ictal activity more in one dataset than the other, the results will be skewed. To make valid comparisons, Halford (2009) explains how an evaluation dataset can help to create more reliable benchmark tests, and in (Halford et al., 2011) and (Halford et al., 2012) a proposed benchmark dataset is introduced for inter-ictal epileptiform spike detection in scalp EEGs. An important finding in (Halford et al., 2011) was the relatively large inter-rater variance (also reported by others, e.g. (Azuma et al., 2003; Benbadis et al., 2009)), which points to an even greater need to use a single, commonly accepted dataset for benchmarking purposes.

Based on our literature review together with the reviews from Halford (2009) and Wilson and Emerson (2002), we have concluded that automated spike detection remains a sought after goal for clinicians given its potential to reduce the time taken by visual inspection alone and also to improve inter-rater reliability. Although many algorithms exist with some having more success than others, improvements are still needed to make them widely accepted in clinical reality.

Our review also pointed out two issues that remain under-emphasized in this field. These are related to performance criteria and user acceptance. First, the current performance measures provide great statistical benchmarks to measure the accuracy and reliability between systems, but often neglects the most important question of all, which is: "How much time and effort will it take the reviewer to use this feature?". For example, a system with a lower false detection rate might lead to less work for the reviewer, but this often translates to a system with lower sensitivities given the typical trade-off between sensitivity and false detection rates for current systems (see chapter 6). In addition, a false detection rate even as low as 0.5 false positives per minute can still require a user to scan through more than one thousand events in long-term recordings given the design of current methods that do not assign certainty or priority to each event. Unfortunately, even the global benchmark dataset described in (Halford et al., 2011) and (Halford et al., 2012) does not take these important factors into account when benchmarking systems. The second under-emphasized issue relates to the complexity and ease of use of currently available systems. Spike detection systems appear complex and difficult to use by the end user. This creates the problem where a reviewer prefers to perform a visual review rather than use an automated method which he does not understand or trust. Although there are more reasons why automated spike detection has not become generally accepted, we conclude that these are two of the main issues that need to be addressed before automated spike detection will become 
widely accepted, and consequently, our main focus will be placed on them.

\section{Objectives and outline of this thesis}

The main objectives of this thesis are to find reliable methods and efficient reviewing techniques that will help with the review and interpretation of routine outpatient EEGs. Our focus is not centered around fundamental neuroscience, but instead on the translational aspect of bringing quantitative EEG analysis closer to neurologists and clinical neurophysiologists in clinical practice. EEGs are widely used and have an important role in neurological examinations. Even though visual analysis has remained best-practice for the better part of the last century, it requires extensive training and has limits due to its time-consuming nature and complexity. An expected outcome of this thesis is to introduce new best practices that will simplify the interpretation of scalp EEG, and thereby improve the consistency and reliability of clinical reviews.

Although many more properties and features are available than those we focus on here, this study investigates the the most common properties described in routine scalp EEGs. Specific aims of this thesis are: (i) finding quantitative measures of the background pattern for automated analysis and a simplified representation of the underlying brain activity, and (ii) detecting inter-ictal epileptiform discharges and presenting them to the reviewer in a time-efficient manner. Both of these tasks have high clinical relevance. In addition to extending existing methods, new quantitative analysis techniques are developed and tested on clinically relevant data. Together with this, novel concepts are presented such as system certainty values and adaptive reviewing algorithms.

An outline of the structure and scope of this thesis is given in Fig. 1.4. Given that our goals are separated into two categories, background activity and transients, this thesis follows the same logical structure. First we present all our work in EEG background analysis (Chapters 2-4), and then proceed to the automated detection of inter-ictal epileptiform discharges (Chapters 5 and 6). Lastly, Chapter 7 provides a summary of this thesis and an outlook into the use (and usefulness) of automated EEG interpretation in clinical practice. A general overview and future perspective is also presented. 
Characterization of the EEG background pattern

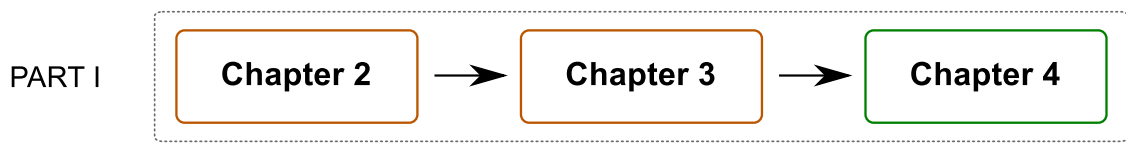

A practical approach to inter-ictal epileptiform spike detection

PART II

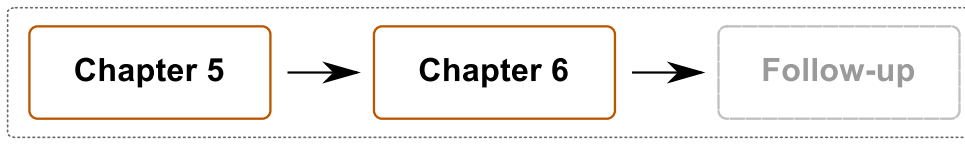

Design and implementation

Clinical evaluation

Stand-alone visual anaysis:

Current best-practice.
Computer-assisted interpretation: Improved consistency, efficiency and reliability.

Fig. 1.4: Outline and scope of this thesis.

\section{PART I: Automated EEG background analysis}

For our goal to automate the interpretation of background activity, the five most commonly reported properties that we found in the diagnostic reports are chosen. These properties are: i) the posterior dominant rhythm and ii) its reactivity, iii) anterior-posterior gradients, iv) asymmetries, and $v$ ) the presence or absence of diffuse slow-wave activity. The first important property is the posterior dominant rhythm (PDR) and its peak frequency. Using an adapted version of the method described by Chiang et al. (2008), a robust and accurate algorithm is developed for the detection of the alpha rhythm in routine EEGs. Chapter 2 describes how this method is implemented and tested. After obtaining accurate estimates of the PDR, other properties such as its reactivity can also be found. Chapter 3 presents the quantitative analysis features we propose to measure the five main properties of the EEG background rhythm. Although the accuracy is very important in automated analysis, a strong focus is also placed on keeping the algorithms simple and open, so that reviewers can spot the weaknesses of the automated methods instead of blindly trusting them. For this, simple guidelines and quantitative features are also included in Chapter 3 to describe how the system evaluates each of the five background properties in the three most commonly used montages. After the methods were developed for automated background interpretation, we set out to evaluate 
the designed system in clinical practice. Chapter 4 describes how 45 routine EEGs are sent to nine experienced electroencephalographers in a multi-center study to evaluate both the accuracy and clinical benefit of using automated interpretation together with visual analysis. Comparisons between reviewers and the system are made using a gold standard and inter-rater agreements.

\section{PART II: Inter-ictal epileptiform spike detection}

Chapter 5 introduces a new approach to inter-ictal spike detection that makes use of a large database of IED template waveforms to find epileptiform activity in the common reference, bi-polar and source montages. This chapter also brings to life the concept of detection certainty for inter-ictal spike detection, and we show that by having a certainty value for each detected event, more likely detections can be separated from events with low likelihoods. In Chapter 6, the use of detection certainty is fully embraced, and it is shown that with this, an adaptive review process can be used to achieve high sensitivities and fast review times simultaneously. 
Part I

EEG background pattern 



\section{Chapter 2}

\section{Automated EEG Analysis: \\ Characterizing the Posterior \\ Dominant Rhythm}

Shaun S. Lodder

Michel J. A. M. van Putten

J Neurosci Meth 2011; 200:86-93 


\begin{abstract}
Automated interpretation of clinical EEG recordings can improve inter-rater agreement in visual reviews and reduce the time required for interpretation. As a first step in the design of a fully automated system, a method is presented to characterize the main properties of the posterior dominant rhythm (PDR), in particular its frequency, symmetry and reactivity. The presented method searches for dominant peaks in the EEG spectra during eyes-closed states with a three-component curve-fitting technique. From the fitted curve, the frequency and amplitude are estimated. The symmetry and reactivity is found using the spectral power at the PDR frequencies. In addition, a certainty value is introduced as a measure of confidence for each estimate. The method was evaluated on a test set of 1215 clinical EEG recordings and compared to the PDR frequencies obtained from the visual analysis, as reported in the diagnostic reports. The calculated PDR frequencies were within $1.2 \mathrm{~Hz}$ of the visual estimates in $92.5 \%$ of the cases. Even higher accuracies were reached when estimates with low certainty values were discarded.
\end{abstract}

Significance: The presented method quantifies an essential feature of the EEG background pattern with a matched accuracy to visual inspection, making it a feasible building block to a fully automated interpretation system. 


\subsection{Introduction}

The electroencephalogram (EEG) is an important technique for the non-invasive recording of brain-related activity. It has a high temporal resolution and can capture both physiological rhythmic activity and other transient processes such as epileptiform discharges. The first human EEG rhythm recorded through the intact scalp was the alpha rhythm (Berger, 1929), and since then, this and other rhythms have been studied extensively. The alpha rhythm plays an important part in many diagnostic fields ranging from depression (Segrave et al., 2010; Spronk et al., 2011) and schizophrenia (Knyazeva et al., 2008; Jin et al., 2006) to Alzheimer's disease (Ishii et al., 2010; Lee et al., 2010) and visual perception (Babiloni et al., 2006; Sewards and Sewards, 1999). It is most visible over the posterior regions during a relaxed state of wakefulness or when the eyes are closed, and its frequency will typically follow a downward gradient from the posterior to anterior region when measured over the scalp (Segalowitz et al., 2010). When measured over the posterior region, the alpha rhythm is also referred to as the posterior dominant rhythm (PDR).

Many attempts have been made to locate and explain the origin of the alpha rhythm, but much is still unknown about its generation and what influence it has on the larger scope of brain function. Instead of having a single generator, the current view is that it is generated by nonlinear interactions of pyramidal cells and modulated by thalamic input and other complex cortico-cortical processes (Steriade et al., 1990; Silva, 1991; Stam et al., 1999; Naruse et al., 2010; Hughes and Crunelli, 2005; Nunez and Ramesh, 2005). The peak frequency for young children typically resides around 3-4 $\mathrm{Hz}$ and gradually increases with age until reaching a maturation point at around $8-13 \mathrm{~Hz}$ when adolescence or young adulthood is reached (van der Stelt, 2008; Marcuse et al., 2008; Segalowitz et al., 2010; Chiang et al., 2011). In adults, a small decrease in frequency with ageing can be observed at a considerably slower rate. The alpha rhythm also shows other important characteristics. It is suppressed when the eyes are opened and becomes again attenuated when the eyes are closed. In some individuals only one dominant peak is visible, whereas for others two distinct peaks can be observed. The reason for splitting is not yet clear, but (Robinson et al., 2001) suggested that nonuniformities in corticothalamic time delays could result in the observed splitting of alpha peaks in some subjects and showed that the mechanism of alpha splitting may be via mode coupling induced by spatial nonuniformities (Robinson et al., 2003).

The alpha rhythm in general has mostly been studied in subject groups of 15 
to 100 individuals and only a small number of studies have used datasets containing one thousand individuals or more (Segalowitz et al., 2010; van der Stelt, 2008; Chiang et al., 2011). In some cases, obtaining characteristics would require the researcher to either read each individual patient report, or to visually inspect every EEG. Aurlien et al. $(1999,2004)$ suggested the use of database structures which are compatible with queries that extract patient information from reports. This approach seems attractive and should be considered when new EEG databases are developed. However, it is not implemented in most existing systems and a description of the PDR for these would require an alternative approach.

In this paper we focus on the characterization of the PDR and we present a method to identify it in EEGs using an automated technique. Dominant frequencies are located in the spectra with a curve-fitting approach and the PDR components are calculated from them accordingly. Using the PDR frequencies, a measure for symmetry and reactivity is also obtained. To provide a confidence score to each characterized rhythm, a certainty value is found and as shown in the results, it makes a powerful contribution to the automated interpretation method.

\subsection{Data and Methods}

\subsubsection{EEG Recordings}

EEG recordings were obtained from the digital EEG database of the department of Clinical Neurophysiology of the Medisch Spectrum Twente (MST) hospital in the Netherlands. All EEGs were recorded as part of the diagnostic process and for most of them a standard 20 minute protocol was used. The protocol included hyperventilation, photic stimulation and eyes-closed states. Standard EEG caps were used with nineteen $\mathrm{Ag}-\mathrm{AgCl}$ electrodes placed according to the international 10-20 system. Electrode impedances were kept below $5 \mathrm{k} \Omega$ to reduce polarization effects, with sample rates of $250 \mathrm{~Hz}$ using a common reference (Brainlab, OSG BVBA).

At the time of this study, the MST database contained EEG records obtained over a period of five years. Only recordings containing annotated events were used and EEGs were only selected if they were categorized as normal brain activity. This was determined by the diagnostic reports from board certified neurologists. The final set of evaluation data consisted of 1215 individuals with ages ranging from a few months to 96 years and contained 611 males and 604 females. 


\subsubsection{Estimation of the PDR Frequency}

Eyes-closed segments were extracted from the EEGs to estimate the frequency of the PDR. The segments were limited to two minutes in length and those that occurred during hyperventilation or photic stimulation were ignored. The remaining segments were split into epochs of five seconds each, and to avoid high-amplitude movement artifacts from interfering, epochs were discarded if they contained values larger than five times the standard deviation of the whole segment. A spectrum was calculated for the remaining set of epochs and the dominant frequency components were located. The component parameters of each epoch were pooled together and clustered, and based on the size and properties of the clusters, estimates for the PDR frequencies were obtained and a confidence value was computed for each estimate. A description for each step of the method is provided below. The description is divided into three parts: dominant peak location, peak value correction, and PDR estimation.

\subsubsection{Dominant Peak Location}

To find the dominant peak components in each epoch, a normalized spectral density $P_{\text {norm }}$ was calculated with the Welch method using a sliding window of two seconds and an overlap of $75 \%$. A common reference montage was used and only the epochs from channels $\mathrm{O} 1$ and $\mathrm{O} 2$ (occipital region) were used. Each window was zero padded to a length of 8 seconds giving $P_{\text {norm }}$ a frequency resolution of $0.125 \mathrm{~Hz}$. The spectrum was log transformed and bounded to the frequency range $\left[f_{\min }, f_{\max }\right]=[3,18] \mathrm{Hz}$

$$
P_{\log }(f)=\log \left(P_{\text {norm }}(f)\right), \quad f \in\left[f_{\min }, f_{\max }\right] .
$$

As discussed in Section 2.1, the PDR is known to have either one or two spectral peaks. Based on this, the log spectrum was assumed to consist of two peak components $P_{\mathrm{pk} 1}$ and $P_{\mathrm{pk} 2}$ and some background processes $P_{\mathrm{bg}}$. Using the log spectra, the Levenberg-Marquardt algorithm (Levenberg, 1944) was used to approximate a 
spectral curve given by:

$$
\begin{aligned}
P_{\mathrm{log}}(f) \approx P_{\mathrm{fit}}(f) & =P_{\mathrm{pk} 1}(f)+P_{\mathrm{pk} 2}(f)+P_{\mathrm{bg}}(f), \\
P_{\mathrm{pk} 1}(f) & =A_{1} \exp \left(\frac{-\left(f-f_{1}\right)^{2}}{\Delta_{1}^{2}}\right), \\
P_{\mathrm{pk} 2}(f) & =A_{2} \exp \left(\frac{-\left(f-f_{2}\right)^{2}}{\Delta_{2}^{2}}\right), \\
P_{\mathrm{bg}}(f) & =B-C \log (f) .
\end{aligned}
$$

Parameters $A_{1}$ and $A_{2}$ define the peak amplitudes, $f_{1}$ and $f_{2}$ their center frequencies, and $\Delta_{1}$ and $\Delta_{2}$ the peak widths. Parameter $B$ defines the normalization of the background component and $C$ a power-law approximation to the background spectrum. If the PDR consisted of one peak only, $P_{\mathrm{pk} 2}$ was discarded by setting $A_{2}$ to zero. Due to the number of free parameters and nature of this curve, localized optimizations could interfere with the approximation. The parameters were therefore estimated in an iterative manner.

Initial values for $B$ and $C$ were calculated first by only fitting the background component to $P_{\log }$ and minimizing the approximation error:

$$
\{B, C\}=\underset{f \in\left[f_{\min }, f_{\max }\right]}{\arg \min }\left|P_{\log }-P_{\mathrm{bg}}\right| .
$$

Using parameters $B$ and $C$ obtained from (2.6), a curve with one peak was fitted to $P_{\log }$ :

$$
\left\{A_{1}, f_{1}, \Delta_{1}, B\right\}=\underset{f \in\left[f_{\min }, f_{\max }\right]}{\arg \min }\left|P_{\mathrm{log}}-P_{\mathrm{pk} 1}-P_{\mathrm{bg}}\right| .
$$

Initial values for $A_{1}$ and $f_{1}$ were calculated from the magnitude and location of the highest peak in $\left(P_{\log }-P_{\mathrm{bg}}\right)$ obtained from (2.6). The initial value of $\Delta_{1}$ was set to 1. While minimizing (2.7), parameters $A_{1}, f_{1}, \Delta_{1}$ and $B$ were free to change, but $C$ was kept fixed.

In a similar manner as before, an estimation for $P_{\log }$ with two peaks was found by minimizing:

$$
\left\{A_{2}, f_{2}, \Delta_{2}, B\right\}=\underset{f \in\left[f_{\min }, f_{\max }\right]}{\arg \min }\left|P_{\mathrm{log}}-P_{\mathrm{pk} 1}-P_{\mathrm{pk} 2}-P_{\mathrm{bg}}\right| .
$$

The estimated parameters from minimizing (2.7) were used as initial values, and starting values for $A_{2}$ and $f_{2}$ were calculated from the magnitude and location 
of the highest peak in $\left(P_{\log }-P_{\mathrm{pk} 1}-P_{\mathrm{bg}}\right)$. The starting value of $\Delta_{2}$ was set to 1, parameters $A_{2}, f_{2}, \Delta_{2}$ and $B$ were allowed to change, and $A_{1}, f_{1}, \Delta_{1}$ and $C$ remained fixed.

Values for all the parameters were obtained after approximating the spectrum in (2.8). However, an additional approximation was made where no parameters were kept fixed:

$$
\left\{A_{1}, A_{2}, f_{1}, f_{2}, \Delta_{1}, \Delta_{2}, B, C\right\}=\underset{f \in\left[f_{\min }, f_{\max }\right]}{\arg \min }\left|P_{\mathrm{log}}-P_{\mathrm{pk} 1}-P_{\mathrm{pk} 2}-P_{\mathrm{bg}}\right|
$$

If the approximation error with the new parameter set was smaller than with the previous parameters, the new set was used instead.

To ensure an accurate approximation of $P_{\log }$ with relevant parameters, a number of evaluations were performed during the iterative process. Firstly, a ratio was calculated between the spectral power in $P_{\mathrm{pk} 1}$ and $P_{\log }$ after one peak was fitted. If $P_{\mathrm{pk} 1}$ contributed to less than $50 \%$ of the power in $P_{\mathrm{log}}$, the spectrum was assumed to be without a dominant frequency component and the epoch was rejected. Also, if $f_{1}$ was not in the range $\left[f_{\min }, f_{\max }\right]$, or $\Delta_{1}$ exceeded a threshold $T H_{\Delta}$, i.e. the peak power was not localized, the epoch was rejected. Secondly, when $P_{\log }$ was approximated with two peaks and $\Delta_{2}>T H_{\Delta}$ or $f_{2}$ was not in the range $\left[f_{\min }, f_{\max }\right]$, the second peak was discarded by setting $A_{2}=0$. The threshold $T H_{\Delta}$ was arbitrarily chosen as 2.5.

\subsubsection{Peak Value Correction}

After approximating a curve and obtaining parameters for each of the peaks in the epochs, an intermediate step was performed to improve the location and amplitude of the detected frequency components. It was found that the iterative approach would robustly locate the dominant frequency components, but their exact amplitudes and frequencies were often inaccurate (see Fig. 2.1(a)). A reasonably simple but effective solution was to search for the peak in $P_{\log }$ around the area of the estimated frequencies. By starting at the estimated frequencies $f_{1}$ and $f_{2}$, the peak estimates were shifted towards the positive gradient on $P_{\log }$ until a local maximum was found. If two peaks were present and both were updated to the same point, one peak was discarded.

After updating the peak parameters, the approximated spectrum was converted from the log domain back to the original domain and the local minima were found 


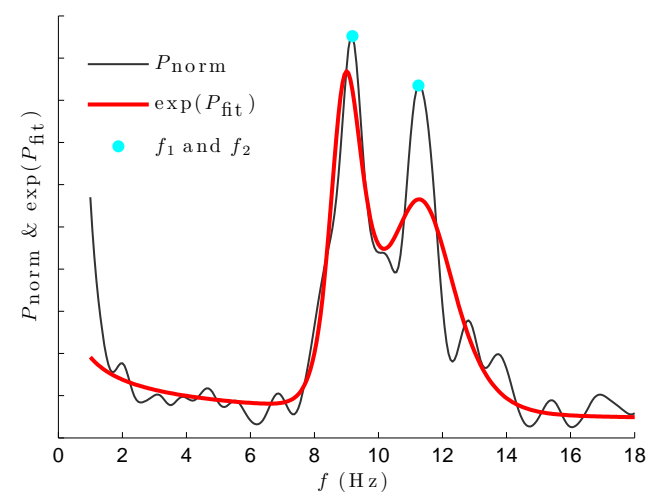

(a)

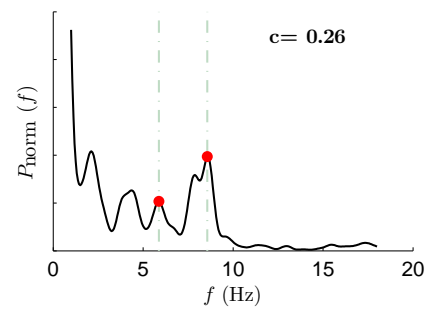

(c)

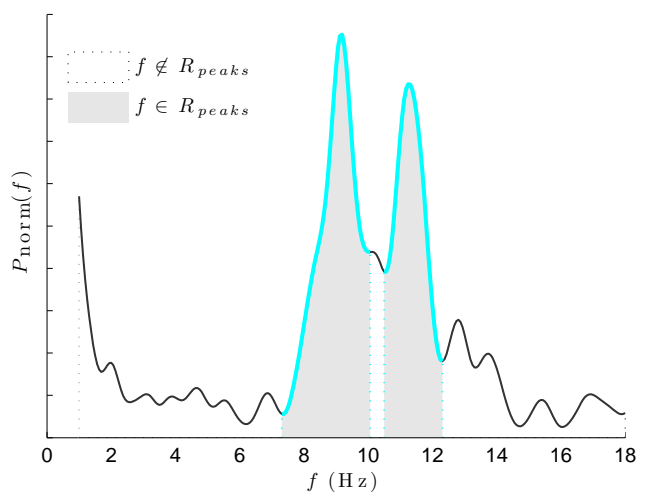

(b)

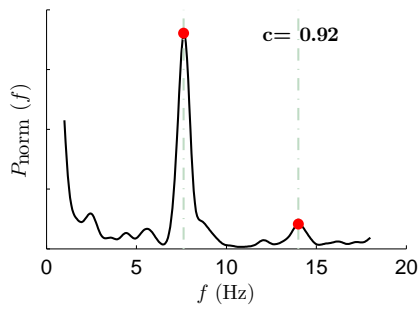

(e)

Fig. 2.1: (a) An iterative curve-fitting technique was used to robustly locate the dominant frequency components from the EEG spectra. An optimization step improved the estimates by searching for the local maxima near the frequencies of the curve components $P_{\mathrm{pk} 1}$ and $P_{\mathrm{pk} 2}$. (b) The peaks were defined to be in the range between their neighbouring local minima. (c)-(e) A correlation parameter was calculated to find the contribution of the spectral peaks to the total spectrum. The examples show how dominant peaks with large amplitudes obtained high values for $c$, whereas less dominant components received low values.

on both sides of each peak. The peak components were assumed to be in the range between the local minima, and to assist in the description of the method, this range is defined as $R_{\text {peaks }} \subseteq\left[f_{\min }, f_{\text {max }}\right]$. An example showing the range is shown in Fig. 2.1(b).

A new spectrum was found from which the peak components were removed:

$$
P_{\text {rem }}(f)=\left\{\begin{array}{ll}
P_{\text {norm }}(f), & f \notin R_{\text {peaks }} \\
\exp (B-C \log (f)), & f \in R_{\text {peaks }}
\end{array},\right.
$$

and to determine the contribution of the peaks to the total spectrum, a parameter 
was calculated based on the correlation between $P_{\text {rem }}$ and $P_{\text {norm: }}$ :

$$
c=1-\operatorname{corr}\left(P_{\text {norm }}, P_{\text {rem }}\right),
$$

where $\operatorname{corr}\left(P_{\text {norm }}, P_{\text {rem }}\right)$ finds the correlation coefficient between $P_{\text {norm }}$ and $P_{\text {rem }}$. If $P_{\text {norm }}$ was noisy or more peak components were present with relatively high peaks, the correlation parameter was low. However, when the peaks contributed to most of the power in $P_{\log }$, a high value for $c$ was obtained. Fig. 2.1(c)-(e) shows examples for different spectra.

\subsubsection{PDR Estimation}

After locating the dominant components in each epoch, the peaks were pooled together and sorted according to frequency. Clusters were formed by grouping peaks together if the frequency difference between them was less than $0.2 \mathrm{~Hz}$, and clusters consisting of one peak or smaller in size than $75 \%$ of the largest cluster were discarded. The remaining set of clusters was defined as $\left\{K_{j}\right\}_{j \in\{1, . . N\}}$, where $K_{j}=\left\{A_{i}, f_{i}, c_{i}\right\}_{i \in\left\{1, \ldots, M_{j}\right\}}$ denoted the amplitudes, frequencies, and the correlation values of the $M_{j}$ peaks in the cluster.

The PDR was assumed to be the largest two clusters in the set and estimates were calculated from them accordingly. If only one cluster was available, the EEG was assumed to contain only one PDR. A weighted average was calculated to obtain the PDR frequency $f_{\mathrm{PDR}}$ and amplitude $A_{\mathrm{PDR}}$ from a given cluster according to:

$$
\begin{gathered}
A_{\mathrm{PDR}}(j)=\sum_{i}^{M} A_{i} \omega_{i}, \\
f_{\mathrm{PDR}}(j)=\sum_{i}^{M} f_{i} \omega_{i}, \\
\omega_{i}=c_{i} / \sum_{k}^{M} c_{k} .
\end{gathered}
$$

As shown in (2.14), the correlation values were used as weights. They were also combined with the size of the cluster to calculate a certainty measure for the PDR 
estimate:

$$
\operatorname{certainty}(j)=\operatorname{erf}(\omega M) \sum_{i}^{M} \frac{c_{i}}{M}
$$

By design, the certainty is a normalized value between zero and one. Low correlation values reflect less dominant components which in turn lead to lower certainty values. Smaller clusters are more likely to produce erroneous estimates and the certainty value is therefore also dependent on the cluster size. The parameter $\omega$ serves as a weight factor to balance the influence between the correlation values and the cluster size component. Through a trial-and-error approach, a value of $\omega=0.005$ was found to be suitable.

After calculating the PDR frequencies and amplitudes for a given EEG, two additional steps were performed: Firstly, if two rhythms were detected by the method and only one was in the alpha frequency range $(8-12 \mathrm{~Hz})$, the outlying rhythm was discarded based on the assumption that it was not part of the PDR. Secondly, if two rhythms were found and both resided outside of the alpha range, the rhythm with the largest amplitude was identified as the PDR and the other was discarded.

\subsubsection{Symmetry and Reactivity}

The symmetry and reactivity was calculated by evaluating the spectral power around the calculated PDR frequencies. For reactivity, two-minute segments of EEG were extracted for both the eyes-closed and eyes-open state over channels $\mathrm{O} 1$ and O2. A spectrum was calculated for each using Welch's method with a window length of 10 seconds and no overlap. The mean power was calculated in a $0.2 \mathrm{~Hz}$ frequency band around the PDR frequencies, and using this the reactivity was defined as:

$$
\text { reactivity }=\frac{P_{\mathrm{EC}}}{P_{\mathrm{EO}}+P_{\mathrm{EC}}},
$$

where $P_{\mathrm{EC}}$ is the mean power for the eyes-closed state and $P_{\mathrm{EO}}$ the mean power for the eyes-open state. The reactivity measure in (2.16) is a normalized value between zero and one. Large suppression of the PDR with eyes opening will result in high reactivity values, whereas smaller changes will indicate lower reactivity.

A measure for symmetry was found by comparing the spectral power between the left and right occipital regions. As with the reactivity, the mean power in a $0.2 \mathrm{~Hz}$ band around the PDR frequencies is calculated. However, this time the mean power 
in channels $\mathrm{O} 1$ and $\mathrm{O} 2$ are not combined, but calculated separately. Using the mean power from the left- and right occipital regions, the symmetry was calculated as:

$$
\text { symmetry }=\frac{P_{\text {right }}-P_{\text {left }}}{P_{\text {right }}+P_{\text {left }}},
$$

where $P_{\text {left }}$ and $P_{\text {right }}$ is the mean power calculated for channels $\mathrm{O} 1$ and $\mathrm{O} 2$ respectively. The symmetry measure is normalized between -1 and 1 . Negative values point to larger PDR amplitudes over the left occipital region and positive values to larger amplitudes over the right.

\subsubsection{Computed Frequencies vs. Visual Estimates}

To evaluate the accuracy of the characterization method, visual estimates of the PDR frequencies were taken from diagnostic reports of the EEGs. One PDR frequency was given in 1089 (89.63\%) of the reports and two in 114 (9.38\%). A PDR estimate was not given in $12(0.99 \%)$ of the EEGs, and in many cases, a second lower peak was also ignored. For some EEGs, the PDR was also not reported at a specific frequency, but instead given as a frequency range due to some observer uncertainty or non-stationarity in the data. Frequency ranges were reported for $640(55.2 \%)$ of the EEGs, and for these the mean width of the visual estimate was chosen as the frequency in the center of the range. The mean width of the reported ranges was $1.28 \mathrm{~Hz}(\mathrm{SD} 0.91 \mathrm{~Hz})$.

To measure the accuracy between the calculated PDR frequencies and the visual estimates, frequency differences between the two for each EEG were calculated according to:

$$
\left\{f_{\text {diff }}\right\}_{p}=\left\{f_{\text {obs }}\right\}_{p}-\left\{f_{\text {cpu }}\right\}_{p},
$$

where $f_{\text {cpu }}$ is the calculated PDR frequency, $f_{\text {obs }}$ is the visual estimate, and $p$ defines the index of the corresponding EEG. If more than one peak was observed with visual estimation, the difference between $f_{\text {cpu }}$ and the nearest $f_{\text {obs }}$ was found. If two PDR frequencies were characterized by the method, a frequency difference for both estimates was found using the nearest visual estimate. The method's accuracy was defined as the percentage of estimates where $f_{\text {diff }}$ had a smaller difference than a given tolerance. Three tolerance values were evaluated: $0.6 \mathrm{~Hz}, 1.2 \mathrm{~Hz}$ and $1.8 \mathrm{~Hz}$. 


\subsection{Results}

Using the described method, PDR estimates were found for 1160 of the 1215 (95.5\%) EEGs. In the remaining $4.5 \%$, there were either insufficient artifact-free epochs to estimate from, or only wide-band spectral components were present and no dominant peaks. During estimation, $5.5 \%$ of the epochs in all EEGs were rejected for having high-amplitude artifacts, and a further $23.9 \%$ were discarded after no dominant peaks were found. Two PDR frequencies were identified in only $20(1.6 \%)$ of the EEGs. Fig. 2.2 shows the number of EEGs grouped by age together with the number in which the PDR was identified. Most of the rejected EEGs (78.2\%) were from the age range between 0 and 5 years.

Figures 2.3(a) and 2.3(b) show the distribution of the observed and calculated frequencies over age respectively. Although more points are present in Fig. 2.3(a) than in Fig. 2.3(b) (see histogram in Fig. 2.2), many are plotted on top of each other due to a lower frequency resolution $(0.5 \mathrm{~Hz})$. A smoothed histogram (blue) was added to indicate the density of the points. The common trend of the PDR frequency over age was calculated by fitting a curve to each set of peaks with a minimum mean squares error fit. A 7-th degree polynomial provided a sufficient fit, and the approximated trends are shown in red in Fig. 2.3(a) and Fig. 2.3(b) respectively. The correlation coefficient between the two polynomials from 0 to 96 years was 0.994 , showing an almost identical trend of the frequencies over age. Fewer outliers are seen in Fig. 2.3(b) compared to Fig. 2.3(a).

A histogram of the frequency differences between visual estimates and characterized PDR frequencies is shown in Fig. 2.4(a). Three tolerance values were evaluated: $0.6 \mathrm{~Hz}, 1.2 \mathrm{~Hz}$ and $1.8 \mathrm{~Hz}$, and accuracies of $75.9 \%, 92.5 \%$ and $96.0 \%$ were obtained for them respectively. The mean frequency difference between visual estimates and calculated frequencies was $0.52 \mathrm{~Hz}$. A scatter plot of $\left\{f_{\mathrm{obs}}, f_{\mathrm{cpu}}\right\}$ for all estimate pairs is shown in Fig. 2.4(b) and a smoothed histogram (blue) is added to indicate the density of the points. The relationship between observed and computerized estimates was calculated as $f_{\mathrm{obs}}=1.00 f_{\mathrm{cpu}}$ (minimum mean square error approximation, fixed offset through zero) with a residual error (root mean square) of 0.96. The correlation between observed and computerized estimates was 0.79 . Fig. 2.4(c) shows the frequency differences of each $\left\{f_{\text {obs }}, f_{\text {cpu }}\right\}$ pair plotted against the certainty of the characterized rhythm. The plot shows clearly that estimates with high certainty values were mostly accurate, whereas inaccurate estimates received certainties below 0.02 . To highlight this, estimates with certainties below 0.02 


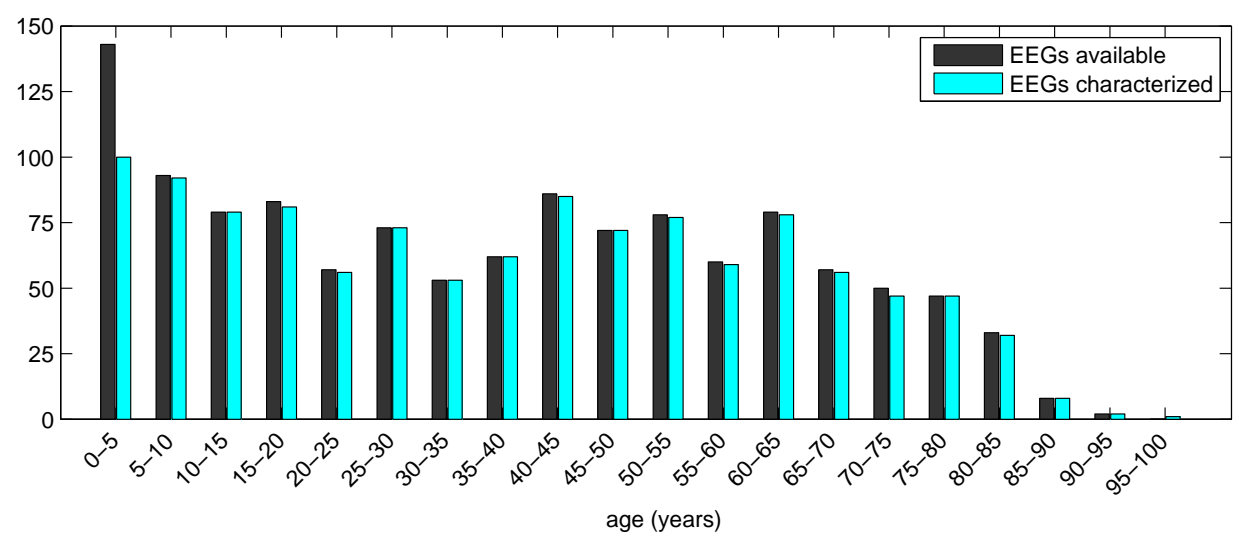

Fig. 2.2: Age distribution of EEGs in the evaluation set. The total number of EEGs is represented in black and the number for which estimates were found in blue. Most of the rejected EEGs were from subjects below 3 years lacking eyes-closed epochs without movement artifacts.

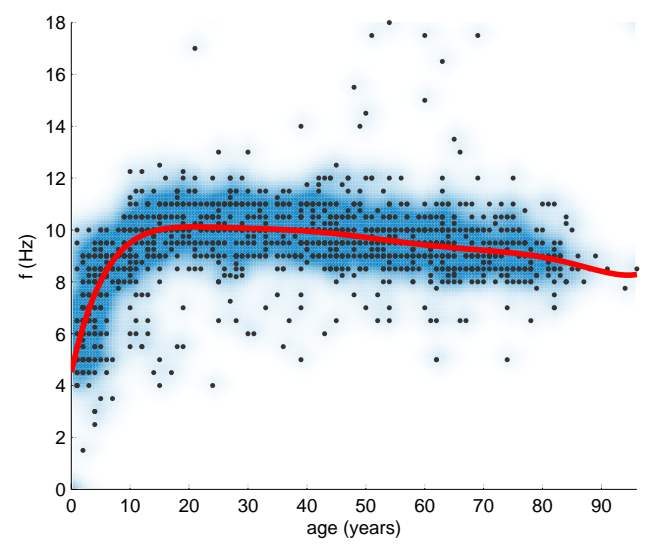

(a)

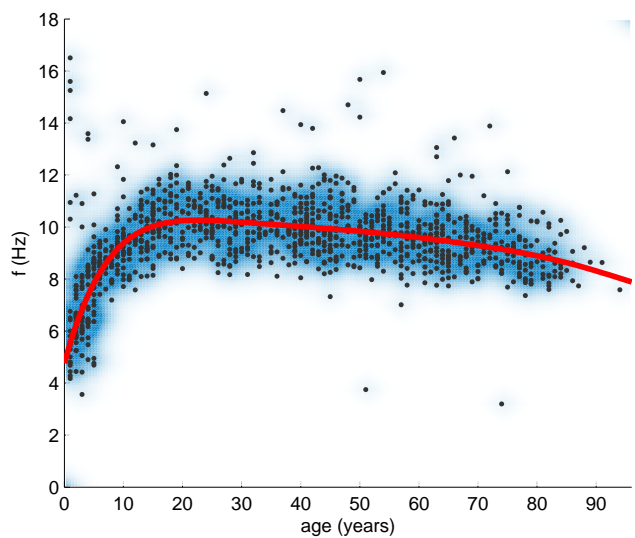

(b)

Fig. 2.3: (a) Visual estimates $\left(f_{\text {obs }}\right)$ and (b) calculated frequencies $\left(f_{\mathrm{cpu}}\right)$ of the PDR in all EEGs over age. The density of the points is shown in blue and a line that approximates the trend over age is plotted in red. The correlation coefficient between the two lines was 0.994 , showing that the calculated frequencies and visual estimates had an almost identical trend over age.

were plotted as red crosses instead of black dots.

After discarding the estimates with low certainty values from the evaluation set, the accuracy of the remaining set increased significantly. Table 2.1 shows the percentage of estimates which were within a tolerable range of the visual estimates. The characterization accuracy was evaluated using the tolerance values of $0.6 \mathrm{~Hz}$, $1.2 \mathrm{~Hz}$ and $1.8 \mathrm{~Hz}$. The first column of Table 2.1 shows the exclusion criteria of the 
Table 2.1: Accuracy of the presented method using three tolerance ranges and discarding estimates below 3 different certainty thresholds. Accuracy was defined as the percentage of estimates where $f_{\text {diff }}=f_{\text {obs }}-f_{\text {cpu }}$ was smaller than the tolerance indicated.

\begin{tabular}{lllll}
\hline & $\left|f_{\text {diff }}\right|<0.6 \mathrm{~Hz}$ & $\left|f_{\text {diff }}\right|<1.2 \mathrm{~Hz}$ & $\left|f_{\text {diff }}\right|<1.8 \mathrm{~Hz}$ & Discarded \\
certainty $>0.0$ & $75.9 \%$ & $92.5 \%$ & $96.0 \%$ & $0 \%$ \\
certainty $>0.02$ & $80.4 \%$ & $96.0 \%$ & $98.4 \%$ & $13.1 \%$ \\
certainty $>0.25$ & $83.9 \%$ & $98.1 \%$ & $99.4 \%$ & $41.0 \%$ \\
\hline
\end{tabular}

Table 2.2: Summary of the characterized PDR properties grouped by age. Values as mean and standard deviation in each group.

\begin{tabular}{lllll}
\hline Age & Frequency $(\mathrm{Hz})$ & Amplitude $(\mu \mathrm{V})$ & Symmetry & Reactivity \\
$0-5 \mathrm{yrs}$ & $7.14 \pm 2.35$ & $11.46 \pm 6.36$ & $0.02 \pm 0.33$ & $0.77 \pm 0.17$ \\
$5-10 \mathrm{yrs}$ & $8.54 \pm 1.17$ & $11.45 \pm 4.41$ & $-0.08 \pm 0.28$ & $0.85 \pm 0.14$ \\
$10-20 \mathrm{yrs}$ & $9.98 \pm 1.13$ & $8.73 \pm 4.34$ & $-0.03 \pm 0.25$ & $0.87 \pm 0.12$ \\
$20-40 \mathrm{yrs}$ & $10.21 \pm 1.02$ & $5.42 \pm 2.99$ & $-0.07 \pm 0.23$ & $0.85 \pm 0.13$ \\
$40-60 \mathrm{yrs}$ & $9.95 \pm 1.20$ & $5.13 \pm 3.21$ & $-0.04 \pm 0.25$ & $0.85 \pm 0.15$ \\
$60-80 \mathrm{yrs}$ & $9.37 \pm 1.10$ & $4.88 \pm 2.70$ & $-0.03 \pm 0.23$ & $0.82 \pm 0.17$ \\
$>80 \mathrm{yrs}$ & $8.68 \pm 1.10$ & $5.03 \pm 2.92$ & $-0.07 \pm 0.26$ & $0.77 \pm 0.20$ \\
\hline
\end{tabular}

evaluation set and the last column the number of estimates which were excluded. In the first row, none of the characterized rhythms were discarded and accuracies of $75.9 \%, 92.5 \%$ and $96.0 \%$ were obtained. The second row shows how by discarding estimates with very low certainty values, a substantial increase in accuracy $(80.4 \%$, 96.0\% and $98.4 \%$ ) can be obtained while still keeping the exclusion rate low (13.1\%). The last row shows that even higher accuracies can be reached $(83.9 \%, 98.1 \%$ and 99.4\%), but at the price of higher exclusion rates (41.0\%).

The key results from evaluating the frequency, amplitude, symmetry and reactivity of the estimated posterior dominant rhythms is summarized in Table 2.2. The EEGs were grouped in suitable age ranges and mean and standard deviation values were found for each property accordingly. As noted before in Fig. 2.3(a) and Fig. 2.3(b), the PDR frequency is lower in younger subjects and increases over age until adulthood is reached. The PDR slows down from the age of 20 years onwards, but at a much slower rate. Higher peak amplitudes are also observed for younger individuals.

The symmetry values show no significant change during ageing and the PDR appears to be fairly symmetrical between the two hemispheres. Reactivity of the 


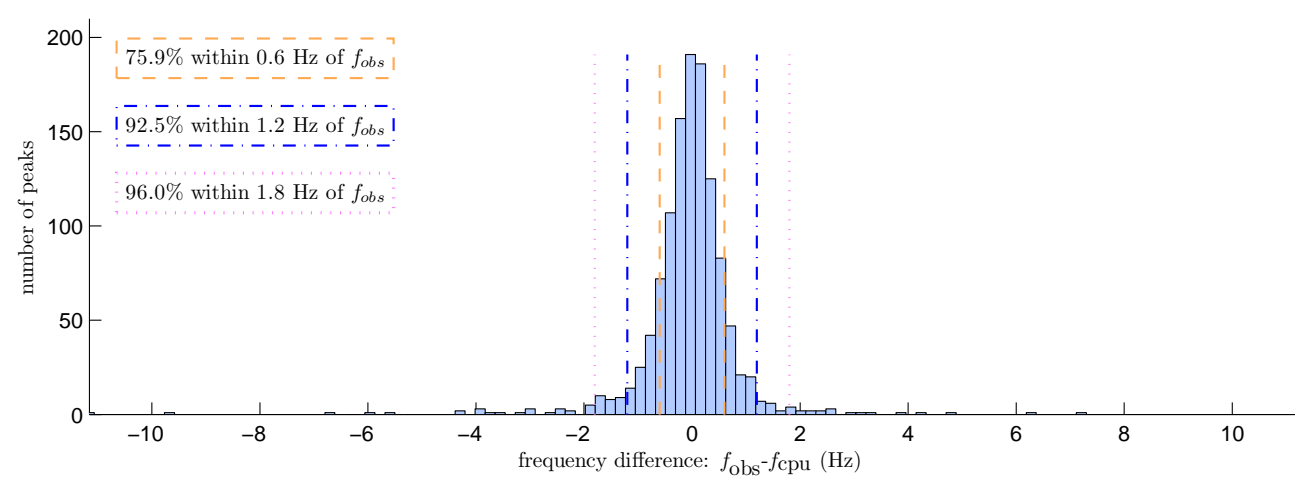

(a)

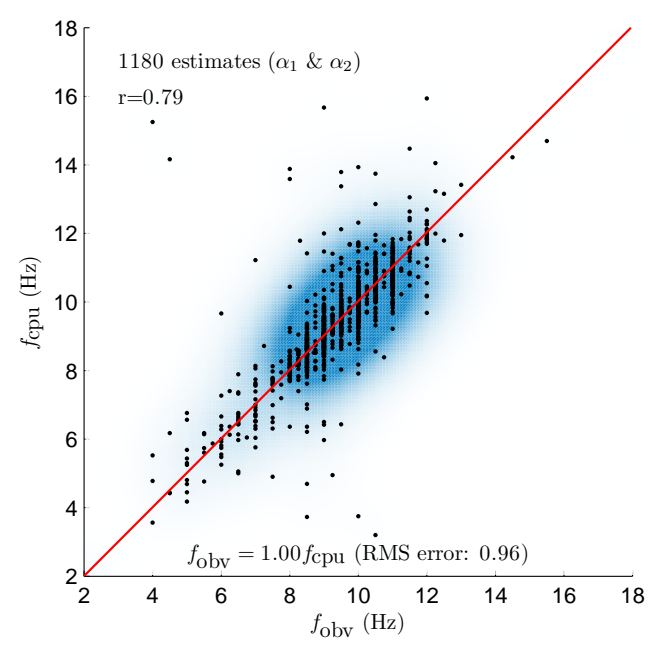

(b)

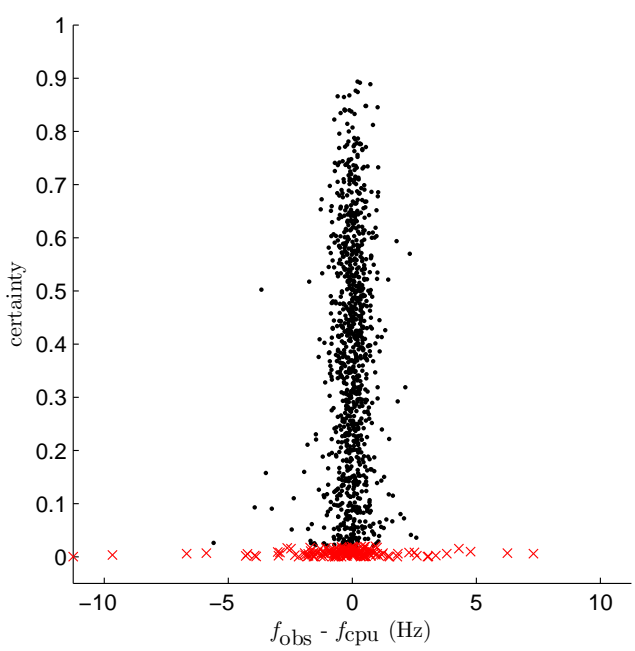

(c)

Fig. 2.4: (a) A histogram showing the frequency difference between visual estimates and calculated frequencies. To view the method's accuracy, three tolerance values were chosen and the number of estimates were counted where frequency differences were smaller than the tolerance values. Boundaries of the tolerances are shown as dotted lines and the accuracies are given in the figure legend. (b) A scatter plot showing $f_{\text {obs }}$ vs. $f_{\text {cpu }}$ for all estimate pairs. The relationship between $f_{\text {obs }}$ and $f_{\text {cpu }}$ is shown in red (line offset through zero). (c) The frequency difference between calculated and visual estimates against the certainty of the calculated rhythm. PDR estimates with certainties below 0.02 are shown as red crosses. Most outliers had very low certainty values.

PDR follows a similar trend over age as the change in frequency. The reactivity increases with age until adulthood is reached, after which it shows a slow decrease from the age of 20 years onwards. Note that for older age groups the variance also increases, indicating that the decrease in reactivity might be accounted for by only some individuals and not the entire group. 


\subsection{Discussion}

Quantitative analysis of EEG finds increasing applications in clinical medicine (Cloostermans et al., 2011; Arciniegas, 2011; Korotchikova et al., 2011; van Putten et al., 2004; van Putten, 2008). Here we introduce a method to automatically characterize the frequency, amplitude, symmetry and reactivity of the PDR together with an accompanying certainty value. With the described method, we were able to estimate the PDR frequency within $1.2 \mathrm{~Hz}$ of the visual estimates in more than $92.5 \%$ of the cases.

In our approach, we use a curve-fitting technique to locate the dominant peaks in the spectrum. By describing the various spectral components with a curve, we were able to find more reliable peak estimates than simply defining the highest peaks in the spectra. This is mainly due to the relatively large variance present in spectral estimates from short time-series, as discussed in e.g. (Broersen, 2006; Thakor and Tong, 2004).

The frequency range of interest was chosen from 3-18 Hz to account for the lower PDR frequencies of younger subjects. Most of the rejected EEGs were from subjects aged between 0 and 5 years (Fig. 2.2), and with further investigation, it was found that in this group the most subjects below 3 years had none or only a few eyesclosed epochs without artifacts. After discarding the contaminated epochs, too few remained to estimate a reliable PDR. Also, no dominant frequency components were found in $23.9 \%$ of the epochs over all EEGs. However, given the condition that the first dominant peak had to contain $50 \%$ of the total power in the spectrum (Section 2.2.2.1), many may have been overseen due to lower amplitudes.

The symmetry and reactivity measures were obtained by evaluating the spectral power at the calculated PDR frequencies. Symmetry and reactivity are highly relevant features in clinical EEG interpretation as they may point to focal ischaemia (van Putten and Tavy, 2004) or neurodegenerative disorders (Babiloni et al., 2010). Also, similar to other diagnostics, the certainty value we added assists the clinician in the relative confidence of particular findings. The certainty value was calculated by evaluating different aspects of the method during the characterization process, and as shown in Fig. 2.4(c), erroneous frequency estimates were mostly characterized with low certainty scores. As further shown in Table 2.1, the accuracy of the system was improved by $\sim 5 \%$ when PDR estimates with low certainties were discarded, leaving PDR frequencies of $98.1 \%$ of the remaining estimates within $1.2 \mathrm{~Hz}$ of the visual estimates. Although most of the erroneous estimates had low certainty 
values, many accurate estimates were also assigned low certainties. This occurred when EEGs had a small number of artifact-free epochs available. Fewer epochs lead to fewer spectra from which the PDR was estimated, which resulted in less reliable estimates and consequently lower certainty values.

It should be noted that the PDR amplitudes do not reflect the true peak amplitude of the posterior dominant rhythm. The estimate is based on the spectra of the EEG which was found using Welch's method with a discrete Fourier transform. As a result of using discrete methods, spectral leakage distorts the measurement in such a way that energy from a given frequency component spreads over adjacent frequency bins. The spectral amplitude is therefore lower than the true peak amplitude.

Although not the primary objective of this study, we found that the peak alpha frequency typically started at $4.5 \mathrm{~Hz}$ and increased with age until maturing around the age of 16 years (Fig. 2.3). A slow decline in frequency was observed from 20 years onwards. This confirms earlier reports on the age dependency of the alpha rhythm as discussed in (van der Stelt, 2008; Marcuse et al., 2008; Segalowitz et al., 2010; Aurlien et al., 2004). The mean PDR frequency over age was also reported by (Aurlien et al., 2004). The similarity of the age-dependency of the PDR is striking, as the differences between their findings (Fig. 2(A) in (Aurlien et al., 2004)) and ours (Fig. 2.3) at all ages are within $\sim 0.25 \mathrm{~Hz}$. In the same study, Aurlien et al. (2004) also reported on inter-observer reliability, showing mean frequency differences of up to $\sim 0.9 \mathrm{~Hz}$ between visual estimates. This is similar to a mean frequency difference of $0.5 \mathrm{~Hz}$ between the visual estimates and calculated PDR frequencies in our study.

The PDR is focussed on the alpha rhythm over the posterior region, and in (Chiang et al., 2008, 2011) a similar technique to characterize the alpha rhythm was explored. In (Chiang et al., 2008) the authors tested their method on 100 subjects (49 females, 51 males) and classified each EEG in one of three categories: (i) no alpha rhythm detected (4 EEGs), (ii) a single alpha rhythm found (48 EEGs) and two alpha rhythm components found (48 EEGs). A comparison to visual estimates was however not made. A follow-up study investigated the age- and sex-related differences of alpha rhythms over a large (1498 subjects) healthy group (Chiang et al., 2011). Similar to our findings, they found that alpha peak frequencies increase with age until adolescence and then slowly declines thereafter. Another finding that correlates with the results shown in Table 2.2 is that the alpha rhythm power declines with age.

The presence of multiple alpha peaks is strongly supported in the literature (Ste- 
riade et al., 1990; Lopes da Silva et al., 1997; Naruse et al., 2010; Klimesch, 1997; Robinson et al., 2003) and possible reasons for this are discussed in Section 2.1. In (Chiang et al., 2008, 2011), two components were found in approximately half of the subjects, although it was not investigated if any of the detected peaks belonged to other rhythms than the alpha rhythm. Our results show that two PDR components were found in $1.6 \%$ of the EEGs. Given however that our method will discard the second rhythm if it falls outside of the range $8-12 \mathrm{~Hz}$, the results do not reflect an accurate number of EEGs with two PDR components. The reason for discarding these peaks was to increase the specificity of our method by minimizing the detection of rhythms that did not belong to the PDR.

Other approaches to quantify the alpha rhythm together with background EEG patterns include model-based filters (Kemp and Blom, 1981), wavelets and multitapers (van Vugt et al., 2007), fuzzy reasoning (Huupponen et al., 2002; Herrmann et al., 2001), non-parametric methods (Brodsky et al., 1999), and multi-dimensional decompositions (Orekhova et al., 2011). Most of these studies only focussed on finding the onset and duration of the rhythm and not on locating the peak frequencies. Our approach provides a more complete characterization of the PDR. With minor modifications, the method can also be extended to locate dominant rhythms over other electrode positions, for example the Mu rhythm over the motor cortex.

None of the studies using automated methods performed a formal comparison between computed and visual estimates. Accuracies in Table 2.1 of up to $99 \%$ shows that the described method is matched to visual estimation, making it a feasible contribution to automated EEG interpretation.

In summary, a robust and automatic method is presented to characterize the posterior dominant rhythm in human EEGs. Apart from removing the subjectivity of visual estimation, it also brings forward an important building block for automated EEG interpretation. 
Chapter 3

\section{Quantification of the adult EEG background pattern}

Shaun S. Lodder

Michel J. A. M. van Putten

Clin Neurophysiol 2013; 124:228-237 


\begin{abstract}
Visual interpretation of EEG is time-consuming and not always consistent between reviewers. Our objective was to improve this by introducing guidelines and algorithms to quantify various properties, focussing on the background pattern in adult EEGs. Five common properties were evaluated: i) alpha rhythm frequency; ii) reactivity; iii) anterio-posterior gradients; iv) asymmetries and v) diffuse slow-wave activity. A formal description was found for each together with a guideline and proposed quantitative algorithm. All five features were automatically extracted from routine EEG recordings. Modified time-frequency plots were calculated to summarize spectral and spatial characteristics. Visual analysis scores were obtained from diagnostic reports. Automated feature extraction was applied to 384 routine EEGs. Inter-rater agreement was calculated between visual and quantitative analysis using Fleiss' kappa: $\kappa=\{$ i) 0.60 ; ii) 0.35 ; iii) 0.19 ; iv) 0.12 ; v) 0.76$\}$. The method is further illustrated with three representative examples of automated reports. Automated feature extraction of several background EEG properties seems feasible. Inter-rater agreement differed between various features, ranging from slight to substantial. This may be related to the nature of various guidelines and inconsistencies in visual interpretation.
\end{abstract}

Significance: Formal descriptions, standardized terminology, and quantitative analysis may help to improve inter-rater reliability in reporting of the EEG background pattern and contribute to more efficient and consistent interpretations. 


\subsection{Introduction}

For almost a century the electroencephalogram (EEG) has been an important and invaluable technique in clinical neurology. Applications include the differential diagnosis of developmental disorders, sleep analysis, and the diagnostic process in epilepsy. Despite tremendous advances in computing power and the availability of digital recordings, the gold standard for the interpretation is still visual analysis. Perhaps the very large variability in EEG patterns, both in physiological and in pathological conditions, limit efforts to automate the diagnostic process. At the same time, the human brain is an expert in visual analysis, including the rejection of artefacts and detection of transients. The processes involved are indeed not trivial to replace by computer analysis (Halford, 2009).

In general, EEG analysis in clinical neurology consists of two parts: analysis of the background pattern and detection of transients (Schomer and Lopes da Silva, 2010; van Putten, 2009). The background pattern can be defined as the mean statistical characteristics of the EEG, and includes features such as the posterior dominant rhythm, reactivity, frequency distribution over the scalp, and the presence or absence of asymmetries. Transients refer to relatively rare events, and include both physiological and pathological waveforms, such as lambda waves, wicket waves or spike-wave discharges.

An accurate interpretation of both the background pattern and the transients is of high importance for correct diagnostics. Unfortunately, various studies have shown that a large inter- and intra-observer variability still exists between reviewers. Depending on the reported feature or decision outcome, the inter-rater agreement (Kappa coefficients) range from slight (0.09) to substantial (0.94) (Haut et al., 2002; Benbadis et al., 2009; Gerber et al., 2008; Azuma et al., 2003). One of the main reasons for this is a lack of consistency in describing the properties accurately. Azuma et al. (2003) showed that by conforming to a set of general guidelines, inter-rater variability could be reduced significantly (Azuma et al., 2003). For many of the EEG properties mentioned however, formal guidelines do not exist or fall short of being used. In addition to this, EEG reports lack consistent terminology to describe the severity of an abnormality.

Apart from improving inter-rater reliability in reports, possibilities exist with computational methods to increase reviewer efficiency and to find characteristics that are hard or even impossible to detect by visual analysis alone. Substantial progress has been made with quantitative methods in the fields of seizure and spike 
detection (van Putten, 2003; van Putten et al., 2005; Kurtz et al., 2009; Halford, 2009; Wilson and Emerson, 2002), but little exists for describing the EEG background pattern quantitatively. Given that background properties provide essential information to the clinician, the use of quantitative tools may be advantageous in assisting with the analysis.

In this paper, we address two issues related to the reviewing of EEGs in a clinical environment. First, we propose guidelines for describing background properties to improve consistency and reduce inter-rater variability. Secondly, by building methods around these guidelines, we introduce quantitative algorithms to measure five of the most commonly reported background properties. The guidelines are kept simple (but feasible) to maximize consensus between reviewers, and the quantitative values are designed intuitively to allow for interpretation in a useful manner. We evaluate our work by comparing reports generated with the quantitative techniques against EEG reports from visual interpretation, and also show two examples where quantitative analysis is used to identify abnormal background patterns.

\subsection{Methods}

\subsubsection{Data}

The dataset for this study was obtained from the department of Clinical Neurophysiology of the Medisch Spectrum Twente (MST). The recordings were made over a period of five years, and for each EEG a standard 20-30 minute recording protocol was used. Electrode impedances were kept below $5 \mathrm{k} \Omega$ to reduce polarization effects, and standard EEG caps were used with nineteen $\mathrm{Ag}-\mathrm{AgCl}$ electrodes placed according to the international 10-20 system. The recordings were made at a sample rate of $250 \mathrm{~Hz}$ using a common reference (Brainlab, OSG BVBA). Technicians annotated eyes open, eyes closed, hyperventillation and photic stimulation events during all recordings.

From the MST database, we selected recordings that were originally made as part of epilepsy investigations. A total of 384 records were used for evaluation, and the patient group consisted of 214 males and 170 females with ages ranging from 18 to 90 years.

For each recording, a diagnostic report written prior to the construction of the proposed guidelines was available. These reports were written by one of two board certified neurologists, and they were used to compare interpretations between visualand quantitative analysis. In order to compare the interpretations, the free text 
Table 3.1: Summary of EEGs described by visual analysis. Descriptions obtained from diagnostic reports.

\section{Alpha rhythm peak frequency}

Normal for age

$303(90 \%)$

Reactivity

Substantial

$301(90 \%)$
Moderate

$26(8 \%)$
Deviating from norm

$33(10 \%)$

Low or absent

$9(3 \%)$

Anterio-posterior gradient

Normal range

$286(74 \%)$
Moderate differentiation

$73(19 \%)$
Abnormal or deviant

$25(7 \%)$

\section{Asymmetries}

None

$295(77 \%)$

At least one

$89(23 \%)$

Diffuse slow-wave activity

Normal EEG Patterns

Diffuse slow waves

$347(90 \%)$

$37(10 \%)$

reports were carefully read and all background properties were categorized by the authors based on a designated set of outcomes for each property. Recordings were excluded if their reports had missing information about any of the properties considered in this study. Table 3.1 shows the outcomes and summarizes the dataset as described by the reports.

\subsubsection{Preprocessing}

An independent component analysis filter was used to reduce the influence of eye blink artifacts on the described features. After calculating the independent components, each was compared to a electrooculogram (EOG) channel recorded together with the EEG. If one of the components showed a substantial correlation with the EOG channel $(>0.3)$, it was removed by setting all its values to zero. The remaining components were projected back to their channel space by applying the inverse transform. No other artifact detection or reduction was performed. 


\subsubsection{EEG features}

We focus on five common background properties: i) the alpha rhythm ${ }^{1}$ and its peak frequency; ii) reactivity; iii) anterio-posterior gradients; iv) asymmetries; and v) the presence or absence of diffuse slow-wave activity. Each of these properties is discussed in the subsections to follow. Other reported background properties include beta activity, lambda waves, changes during hyperventilation, and driving responses during photic stimulation. These properties were however not investigated.

Each subsection below starts with an outline of a property, followed by a proposed guideline to evaluate or describe it, and then followed by a quantitative analysis approach to evaluate the property in a computational manner. Depending on the quantitative feature calculated, either a common reference, Laplacian, or bipolar montage was used. Annotations were extracted in an automated manner to determine eyes open and eyes closed states, and segments during hyperventilation and photic stimulation were ignored. Detailed descriptions of the quantitative algorithms are presented in 3.4.

\subsubsection{Alpha rhythm frequency}

The alpha rhythm was the first rhythmic activity measured in the brain and has been shown to have diagnostic value in diseases ranging from depression (Segrave et al., 2010; Spronk et al., 2011) and schizophrenia (Knyazeva et al., 2008; Jin et al., 2006) to Alzheimer's disease (Ishii et al., 2010; Lee et al., 2010) and visual perception (Babiloni et al., 2006; Sewards and Sewards, 1999). The alpha rhythm is most visible over the posterior regions during a relaxed state of wakefulness when the eyes are closed, and its frequency follows a downward gradient from the posterior region anteriorly when measured over the scalp (Segalowitz et al., 2010). Its peak frequency increases with age until maturation is reached, which typically occurs during adolescence or young adulthood (van der Stelt, 2008; Marcuse et al., 2008; Segalowitz et al., 2010). Thereafter it remains constant, or in some cases, decreases slowly with ageing (Lodder and van Putten, 2011). Decreasing alpha rhythm frequencies with ageing have been suggested to relate to mental deterioration (Gaál et al., 2010).

\footnotetext{
${ }^{1} \mathrm{~A}$ more general name is the posterior dominant rhythm, as in pathological situations the peak frequency may be outside the alpha frequency range. Here, we will further denote it as the alpha rhythm.
} 
In various handbooks, it is stated that a posterior dominant rhythm of less than 8 $\mathrm{Hz}$ during wakefulness in an adult is abnormal (Levin and Lüders, 2000). In healthy individuals, even in the seventh and eighth decade, the mean is maintained at or above $9 \mathrm{~Hz}$ (Obrist, 1976). In a previous study, we found this also to be true in a dataset of 1215 normal EEGs (Lodder and van Putten, 2011). For children however, alpha peak frequencies can be normal below $8 \mathrm{~Hz}$.

Guideline 1: The alpha rhythm frequency is defined as the peak frequency in a spectrogram taken over the posterior region during the eyes-closed state. For a guideline to take age dependency of the peak frequency into account, we propose to use the mean trend over age for normal EEGs, as noted by van der Stelt (2008), Segalowitz et al. (2010), Aurlien et al. (2004), and Lodder and van Putten (2011). Table 3.2 provides a reference derived from the mean trend approximated by Lodder and van Putten (2011), and we consider the alpha rhythm to deviate from the norm (i.e. too slow or fast) if the peak frequency differs by more than $1.8 \mathrm{~Hz}$ from the reference value for a given age.

Automated analysis: To find the alpha rhythm frequency with an algorithmic approach, a method previously described in (Lodder and van Putten, 2011) is used (summarized in 3.4.2). Using a common reference montage, the technique identifies dominant frequency components between $3-18 \mathrm{~Hz}$ in the occipital region. This is done by fitting a curve to the log spectrum of localized segments of the EEG when the eyes are closed:

$$
P_{\log }(f) \approx P_{\mathrm{fit}}(f)=P_{\mathrm{pk} 1}(f)+P_{\mathrm{pk} 2}(f)+P_{\mathrm{bg}}(f)
$$

Peak parameters from the localized segments are clustered together based on frequency similarity, and an alpha rhythm estimate is obtained by finding the mean frequency of the largest cluster. If there are no dominant peaks in the EEG spectra, the method assumes that an alpha rhythm is not present. After estimating the alpha rhythm peak frequencies, they are categorized using Guideline 1 and Table 3.2 as reference.

\subsubsection{Alpha rhythm reactivity}

Reactivity is known as an attenuation of rhythmic activity, occurring mostly in the alpha band, when the brain receives an external stimulus after an idle state 
Table 3.2: Reference to accepted normal alpha rhythm peak frequencies over age. From 0-15 years, the peak frequency is strongly related to age.

\begin{tabular}{lr}
\hline Age & Frequency $(\mathrm{Hz})$ \\
$0-1$ yrs & $5.3 \pm 1.8$ \\
$2-3$ yrs & $6.8 \pm 1.8$ \\
$4-5$ yrs & $7.9 \pm 1.8$ \\
$6-7$ yrs & $8.7 \pm 1.8$ \\
$8-15$ yrs & $9.5 \pm 1.8$ \\
$16-50$ yrs & $9.9 \pm 1.8$ \\
$>51$ yrs & $9.1 \pm 1.8$ \\
\hline
\end{tabular}

(Schomer and Lopes da Silva, 2010). The stimulus can range from eyes opening to auditory input or pain. Reactivity becomes weaker during drowsiness as compared to a fully awake state, and the level of suppression varies with age (Gaál et al., 2010). Although having less diagnostic value than other background properties, the reactivity is known to be lower in demented patients (van der Hiele et al., 2007; Babiloni et al., 2010). Furthermore, it is believed that lower reactivity reflects a reduction of neuronal interconnectivity and a weaker level of neurotransmission, which may be seen in EEGs of the elderly (Gaál et al., 2010). In previous studies by Logi et al. (2011), Douglass et al. (2002), and Ramachandrannair et al. (2005), the prognostic value of reactivity in comatosed patients was also explored with positive results.

Although reactivity is most commonly associated with the suppression of alpha power, a formal definition to quantify it with a scale of normality was not found in existing literature. Most studies compare the alpha power in an idle state to that of a non-idle state, as done for example by van der Hiele et al. (2007), Könönen and Partanen (1993), and Partanen et al. (1997).

Guideline 2: To categorize reactivity, we compare the difference in alpha power over the occipital region between an idle (relaxed, eyes closed) and non-idle (eyes open) state. Suppression of more than $50 \%$ alpha power is considered substantial, between $10-50 \%$ as moderate, and anything less as low or absent.

Automated analysis: Using the alpha frequency obtained by the technique described in Section 3.2.3.1, reactivity is calculated by comparing the difference in al- 
pha power between eyes-open and eyes-closed states. The states are determined by evaluating annotations in the EEG. Using a narrow band around the alpha rhythm peak frequency, reactivity is calculated as

$$
Q_{\mathrm{REAC}}=1-\frac{P_{E O}}{P_{E C}}
$$

where $P_{E O}$ is the mean occipital power in a $0.5 \mathrm{~Hz}$ frequency band when the eyes are open and $P_{E C}$ the mean occipital power in that band when the eyes are closed. The reactivity is classified as substantial when $Q_{\text {REAC }}>0.5$, moderate for $0.1<Q_{\mathrm{REAC}}<0.5$, and low or absent if $Q_{\mathrm{REAC}}<0.1$.

\subsubsection{Alpha power anterio-posterior gradient}

Rhythmic activity from a normal wake brain should be distributed with an anteriorto-posterior gradient over the scalp: higher frequency beta activity with low voltages more prominently over the frontal regions fading posteriorly, and slower waves (e.g. alpha and mu rhythm) with higher voltages over the parietal and occipital lobes (Schomer and Lopes da Silva, 2010). Drowsiness and sleep causes attenuation of the alpha rhythm and slowing of the background rhythm, together with more prominent alpha and theta activity anteriorly. Medication (e.g. benzodiazepines) and anaesthesia (e.g. propofol) can also play a large role in the distribution of rhythmic activity, making it important to evaluate the gradient within clinical context to avoid misinterpretation. Abnormalities in the gradient can point to disorders such as schizophrenia and dementia (Knyazeva et al., 2008; Stevens and Kircher, 1998).

In the EEG reports reviewed, the anterio-posterior gradient described was mainly on alpha band activity. To have clinical relevance, our proposed guideline therefore also focusses on the alpha power distribution.

Guideline 3: Using a Laplacian montage, the gradient is categorized as 'within normal range' if the alpha power distribution is most prominent over the posterior region, 'moderate differentiation' when it becomes evenly distributed over the scalp, and 'abnormal or deviant' if more power is present anteriorly than posteriorly.

Automated analysis: Based on the center-of-gravity feature in (van Putten, 2008), the mean power in the alpha band during an eyes-closed state is calculated 
and a normalized anterio-posterior power ratio is found:

$$
Q_{\mathrm{APG}}=\frac{P_{\mathrm{ant}}}{P_{\mathrm{ant}}+P_{\mathrm{pos}}} .
$$

Here, $P_{\text {ant }}$ and $P_{\text {pos }}$ represent the mean alpha power over the anterior and posterior regions respectively (see 3.4.3). Using this ratio, the alpha power gradient is considered within normal range if $Q_{\mathrm{APG}}$ is lower than 0.4 , moderately differentiated between 0.4 and 0.6 , and abnormal or deviant for values above 0.6.

\subsubsection{Asymmetries}

Asymmetry of the occipital alpha rhythm voltage is present in about $60 \%$ of healthy adults (Maulsby et al., 1968). Only $17 \%$ of healthy adults show voltage differences larger than $20 \%$, and a mere $1.5 \%$ show differences larger than $50 \%$ (Maulsby et al., 1968). As a rule therefore, interhemispheric differences in amplitude larger than 50\% are considered abnormal (Levin and Lüders, 2000; Maulsby et al., 1968). A significant $(>50 \%$ ) voltage asymmetry of the mu rhythm or temporal alpha activity are often observed in healthy adults; a presence of these findings should therefore be interpreted with caution, as this is common in asymptomatic persons (Ebersole and Pedley, 2003). Frequency asymmetries of $1 \mathrm{~Hz}$ or larger also indicate pathology.

Guideline 4: Asymmetrical background patterns are found by comparing rhythmic activity between the two hemispheres in corresponding channel pairs. Reported asymmetries should refer to either $a$ ) a significant amplitude difference between corresponding channels larger than $50 \%$, or $b$ ) an abnormal frequency difference (or absence) of rhythmic components in excess of $0.5 \mathrm{~Hz}$ (which, for a frequency resolution of $0.5 \mathrm{~Hz}$, indicates a difference of $1 \mathrm{~Hz}$ or more).

Automated analysis: Using a Laplacian montage, EEG channels are paired based on symmetrical opposites over the left and right hemispheres:

$$
\begin{aligned}
C_{\{\mathrm{L}, \mathrm{R}\}}=\{ & \{F p 1, F p 2\},\{F 7, F 8\},\{F 3, F 4\},\{T 3, T 4\}, \\
& \{C 3, C 4\},\{T 5, T 6\},\{P 3, P 4\},\{O 1, O 2\}\} .
\end{aligned}
$$

Using the same principle as described by van Putten (2008), a spectral difference is 
calculated for each channel pair. If the EEG shows spectral asymmetry, it will be reflected in the calculation:

$$
Q_{\mathrm{ASYM}}\left(c_{\{L, R\}}\right), c_{\{L, R\}} \in \boldsymbol{C}_{\{\mathrm{L}, \mathrm{R}\}} \cdot
$$

In (3.5), each $c_{\{L, R\}}$ represents one of the channel pairs, and $Q_{\mathrm{ASYM}}\left(c_{\{L, R\}}\right)$ finds a normalized value to quantify the spectral difference between two channels (see 3.4.4). Using a threshold of 0.5 , the EEG is considered to have an asymmetry for any pair that exceeds that value.

\subsubsection{Diffuse slow-wave activity}

Diffuse slow-wave activity is hardly present in healthy waking adults (Ebersole and Pedley, 2003). Normal variants exist, although in most cases its presence points to an abnormality that can result from a number of causes. One common cause is diffuse cortical injury such as anoxia (Cloostermans et al., 2011). Slow-wave activity can also be the result of pharmacological effects such as sedatives or anaesthetic medications (San-juan et al., 2010; Blume, 2006).

Guideline 5: Diffuse slow-wave activity results in increased power over the theta and delta bands and decreased power in the alpha and beta bands. Using this as a guideline, the EEG is considered to contain diffuse slow-wave background activity if less than one third of the power in a spectrogram resides above $8 \mathrm{~Hz}$.

Automated analysis: Using the guideline above, the mean spectrum of the EEG is calculated and the power ratio between $P_{\text {low }}=\{2 . .8\} \mathrm{Hz}$ and $P_{\text {wide }}=\{2 . .25\} \mathrm{Hz}$ is obtained (3.4.5):

$$
Q_{\mathrm{SLOWING}}=P_{\text {low }} / P_{\text {wide }}
$$

For $Q_{\text {SLOWING }}>0.6$ (i.e. less than $40 \%$ of power above $8 \mathrm{~Hz}$ ), too much slow-wave activity is present and the EEG is categorized as abnormal. EEGs with $Q_{\text {SLOWING }}<0.6$ are considered to have sufficiently fast rhythmic activity.

\subsubsection{Evaluation}

To evaluate the value of the proposed guidelines and quantitative measures, a comparison was made between the described properties from the EEG reports and the interpretations made by automated analysis. As described above and also 
shown in Table 3.1, each property was designated by either two or three categories, depending on its relevance. To compare the proposed quantitative measures against visual analysis, three measures were used: sensitivity, specificity, and Fleiss' kappa coefficient (Fleiss, 1971).

Although a common performance measure in quantitative analysis, sensitivity and specificity is defined for two-class problems. It also assumes that the true labels are accurate in describing the property at hand, and does not consider interand intra-rater variability. Regardless, these measures can provide valuable information relating to the development of quantitative analysis features. Sensitivity and specificity are calculated for the three properties with a dichotomous outcome: alpha rhythm frequency, reactivity, and the presence or absence of asymmetries.

Given that our goal is to assist the reviewer with analysis of the background pattern, measuring and maximizing the inter-rater agreement between visual and quantitative analysis is of high importantance. Fleiss' kappa coefficient measures inter-rater agreement in properties with multiple outcomes, and is therefore chosen to achieve this.

\subsection{Results}

A dataset containing 384 adult EEGs was used to compare the proposed guidelines and automated analysis to visual interpretations. Results from individual properties are provided in succeeding subsections. Table 3.3 summarizes the agreement for each property, and Section 3.3.6 gives three examples of how automated analysis can assist in visual interpretation.

\subsubsection{Alpha rhythm frequency}

Using Guideline 1, substantial agreement $(\kappa=0.60)$ was obtained with visual interpretation in determining if the alpha rhythm frequency was within normal range. The quantitative measure could detect abnormal alpha frequencies with high specificity (0.96), although a lower sensitivity (0.64) was reached. The alpha rhythm could not be identified for 48 of the EEGs with quantitative analysis; either because none was present, or because there was not a significant peak amplitude in the power spectrum. Visual interpretation for these 48 EEGs also ranged from no alpha rhythm observed to a weak but visible peak. The results for alpha rhythm frequency and reactivity in Table 3.1 and Table 3.3 only reflect the EEGs in which the rhythm was identified in both visual and quantitative analysis. The mean fre- 
quency difference between reported and calculated peak frequencies was $0.42 \mathrm{~Hz}$ $(\mathrm{SD} \pm 0.66 \mathrm{~Hz})$. The peak frequency reported by visual analysis was often described using a narrow frequency range (mean width $0.57 \mathrm{~Hz}$ ) instead of a single value. In such cases, the frequency difference with quantitative analysis was calculated using the center frequency of that range.

\subsubsection{Alpha rhythm reactivity}

Using the calculated peak frequencies, reactivity was calculated for the 336 EEGs with known alpha rhythms. Reactivity of the rhythm after opening or closing the eyes was assigned to one of three categories: substantial, moderate, and low or absent. Using these categories, a fair agreement of $\kappa=0.34$ was reached.

\subsubsection{Alpha band anterio-posterior gradient}

The quantitative measure for an anterio-posterior gradient showed the lowest agreement of all properties with visual interpretation $(\kappa=0.19)$. It should however be noted that it was the most difficult property to categorize from the EEG free text reports due to inconsistent terminology and an unclear border between normal and deviating differentiation.

\subsubsection{Asymmetries}

Although it adds significant value to report in which region asymmetries exist, our comparison focussed first on accurately identifying EEGs with abnormal asymmetries. The agreement between visual interpretation and automated analysis was low $(\kappa=0.12)$. The sensitivity and specificity for this property were 0.16 and 0.97 respectivrely. No distinction was made between slight and severe asymmetries.

\subsubsection{Diffuse slow-wave activity}

To categorize the presence or absence of diffuse slow-wave activity in the background pattern, our algorithm compared the power in the alpha and beta bands to lower bands. As shown in the last row of Table 3.3, this approach obtained substantial agreement with visual interpretation $(\kappa=0.76)$, high specificity $(0.98)$, and a moderate sensitivity (0.78). 
Table 3.3: Quantitative analysis compared to visual interpretation. Comparisons are made based on automated analysis and the outcome in EEG reports. For properties with a dichotomous outcome, sensitivities and specificities are given in the third and fourth columns. Inter-rater agreement, calculated as kappa coefficients, are shown in the last column.

\begin{tabular}{lllll}
\hline Background property & Quantity & Sensitivity & Specificity & Agreement \\
Alpha rhythm frequency & $Q_{\text {ALPHA }}$ & 0.64 & 0.96 & $\kappa=0.60$ \\
Alpha rhythm reactivity & $Q_{\text {REAC }}$ & - & - & $\kappa=0.34$ \\
Anterio-posterior gradient & $Q_{\text {APG }}$ & - & - & $\kappa=0.19$ \\
Asymmetries & $Q_{\text {ASYM }}$ & 0.16 & 0.97 & $\kappa=0.12$ \\
Diffuse slow-wave activity & $Q_{\text {SLOWING }}$ & 0.78 & 0.98 & $\kappa=0.76$ \\
\hline
\end{tabular}

\subsubsection{Automated analysis assisting visual interpretation in practice}

Some examples are given to illustrate the added value of automated analysis during an EEG review. Using quantitative analysis performed with the described algorithms, automatically generated reports for three EEGs are presented in Fig. 3.1-3.3. At the top of each figure is a summarized outcome of the quantitative analysis written in free text, and below are images to display various EEG properties over time. The images correspond to quantitative features described in Section 3.2.3, with the exception of not reducing a feature to a single value by averaging over frequency or time. These examples clearly illustrate the added benefit of a quantitative system: outlining the background rhythm of the whole recording on a single page in a clear and simple manner.

For the first example (Fig. 3.1), an EEG which was considered normal by both visual analysis and quantitative analysis is shown. Fig. 3.1(b) shows the "assumed" states (i.e. eyes open, eyes close, hyperventilation etc.) of the patient at each time point. This is obtained by parsing the annotations made during recording. By using Fig. 3.1(b) as context, the reviewer can easily compare changes in properties in the figures below it when switching from states. The second image row, Fig. 3.1(c), shows a spectrum of the EEG over time, averaged over all channels. Reactivity of the alpha rhythm is clearly visible when comparing the spectrum in Fig. 3.1(c) to the eyes-open and eyes-closed states in Fig. 3.1(b). Fig. 3.1(d) shows the average symmetry over all left-right channel pairs. For this figure, an even symmetry was reported and as seen in the plots, the symmetry appears to be relatively equal. The last row, Fig. 3.1(e), shows the anterio-posterior distribution of rhythmic activity. As expected for a normal EEG and discussed in Section 3.2.3.3, higher frequency beta activity is seen most prominently over the frontal regions fading posteriorly, 


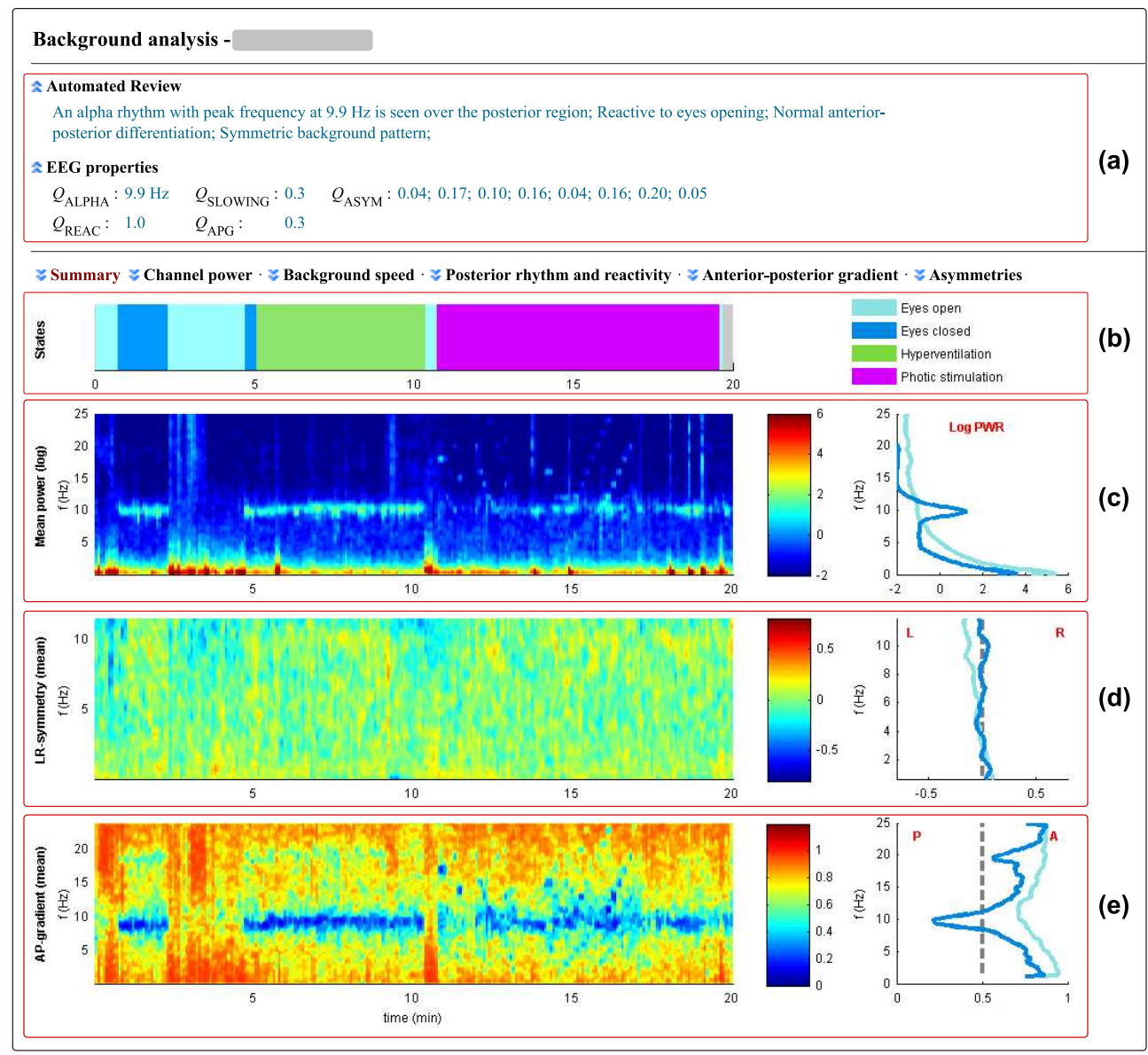

Fig. 3.1: An automated EEG report with all background properties described as normal. (a) Quantitative values (Section 3.2.3) are provided together with an automated free-text description. (b) EEG states (i.e. eyes-open, eyes-closed, hyperventillation etc.) are shown to place quantitative feature plots in correct context. (c) Time-varying spectrum averaged across all channels. (d) Mean left-right symmetry, calculated by averaging Eq. (3.21) over all channels. (e) Time-varying anterio-posterior distribution of rhythmic activity. Note the photic driving responses also visible in (c) and (e).

and slower waves (e.g. alpha and mu rhythm) more towards the posterior region.

For the second example (Fig. 3.2), the EEG background properties were all described as normal, except for a right-dominant alpha asymmetry. This description was based on visual inspection. Quantitative analysis also found all background properties except for asymmetries to be normal. For this EEG, visual inspection and quantitative analysis produced the same outcome. The plots in Fig. 3.2 also show that all properties appear normal, except for a right-dominant asymmetry in 


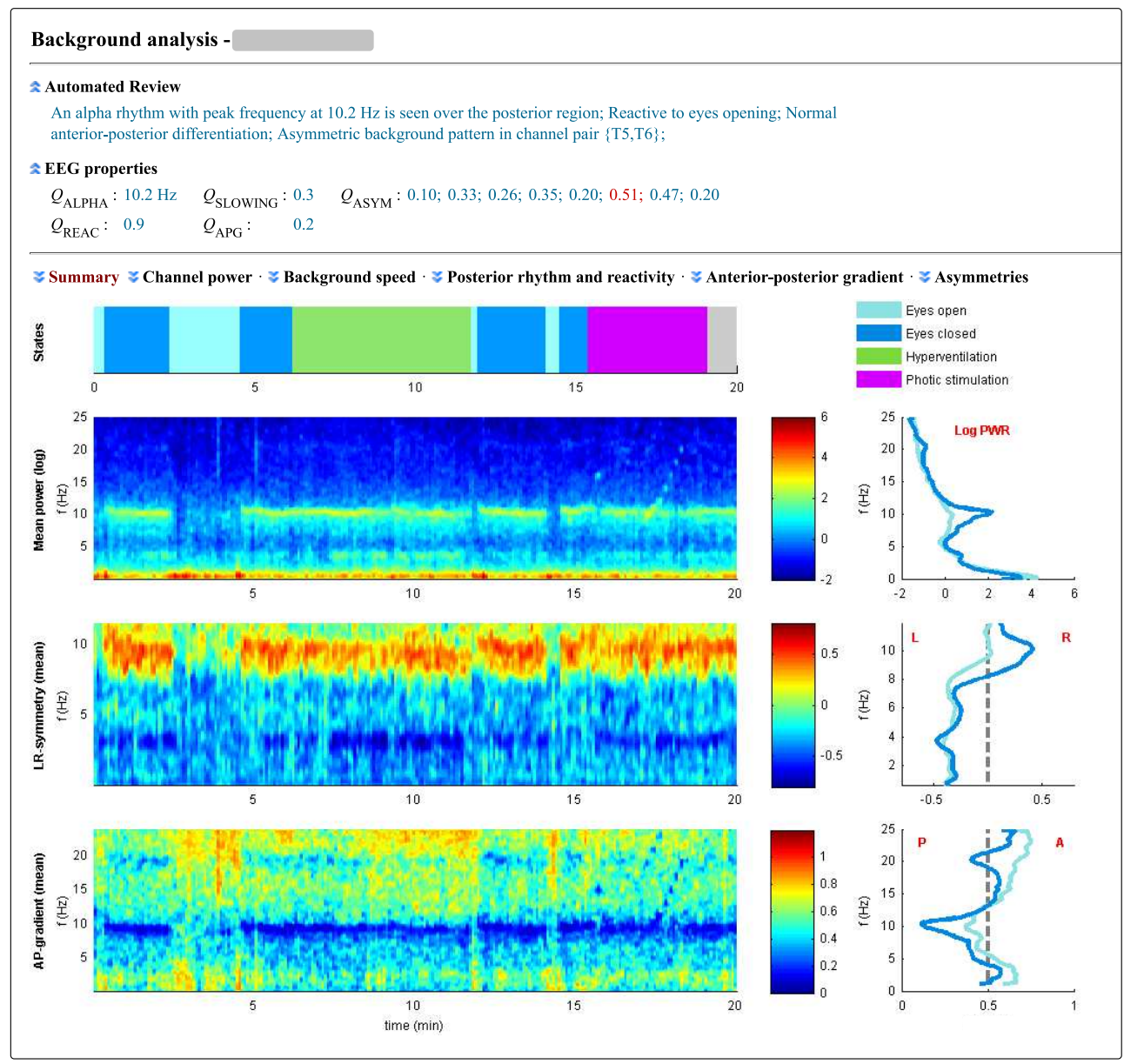

Fig. 3.2: An EEG with all background properties described as normal by visual analysis except for asymmetries over temporal and parietal regions. $Q_{\mathrm{ASYM}}$ shows two channel pairs above threshold, indicating asymmetries found by quantitative analysis. By looking at the left-right symmetry plot, the asymmetry is also clearly presented to the reviewer in a visual manner.

the alpha band and a left-dominant asymmetry in the lower bands.

The third example (Fig. 3.3) shows an EEG where multiple abnormalities occur. Based on visual inspection, the report described it as containing diffuse slow-wave activity, very low or absent reactivity, and an abnormal anterio-posterior gradient. Automated analysis described the EEG to have diffuse slow-wave activity, moderate reactivity, and an abnormal or deviant anterio-posterior gradient. Once again, the plots in Fig. 3.3 can assist the reviewer by providing a time-varying summary of the background properties investigated. As an example, compare the fourth row 


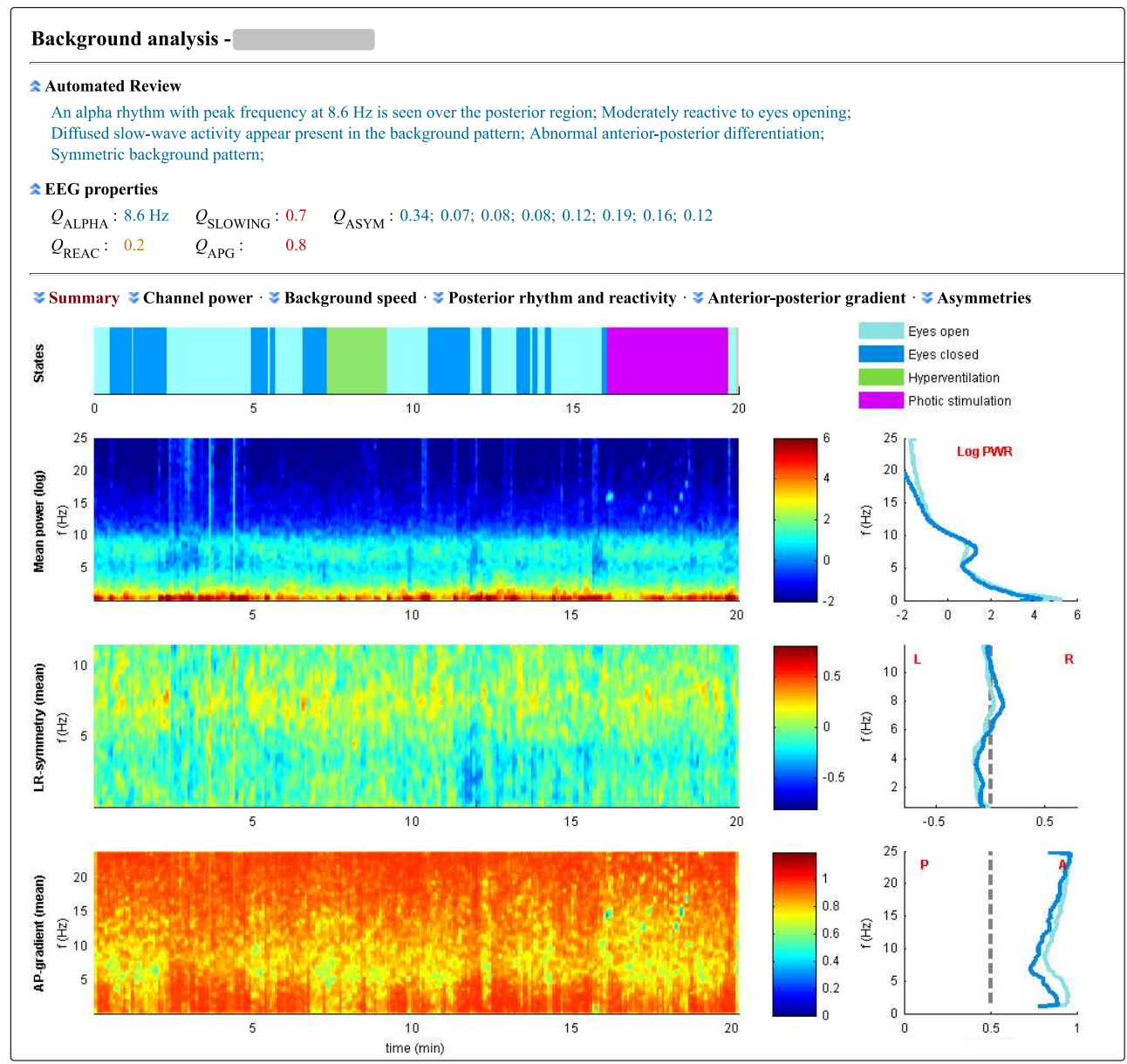

Fig. 3.3: An EEG with several abnormalities reported by visual analysis: diffuse slow-wave activity, moderate to low reactivity and an abnormal anterio-posterior gradient. The quantitative features $Q_{\text {SLOWING }}, Q_{\text {REAC }}$, and $Q_{\text {APG }}$ show that automated analysis comes to a similar conclusion. As visual assistance and an outline of the full recording, plots show too much slow-wave activity and no reactivity in the power spectrum between eyes-open and eyes-closed states. Also, an abnormal distribution of rhythmic activity in the bottom row is seen.

images of Fig. 3.2 and Fig. 3.3: As expected for normal differentiation in Fig. 3.1-3.2, rhythms in the alpha and theta bands appear posteriorly, whereas faster rhythms are more visible anteriorly. Fig. 3.3 however shows that all rhythmic activity appear stronger towards the anterior, corresponding to the EEG report that an abnormal or deviant anterior-posterior gradient exists. 


\subsection{Discussion}

Current methods of reviewing routine EEGs are based mostly on visual analysis of the raw signals. Although this approach still holds the highest specificity and sensitivity for finding abnormalities, it is time consuming and requires extensive training. Apart from this, visual analysis is susceptible to reviewer bias (Haut et al., 2002; Benbadis et al., 2009; Gerber et al., 2008; Azuma et al., 2003). Automated quantification techniques reduce the time required for reviewing and improve consistency in reporting. They can quantify various properties in EEG and even point out abnormalities if found. However, because external factors (e.g. medication or structural damage) may influence the EEG patterns, final interpretation of the findings is always left to the reviewer.

For quantitative analysis of the background pattern, it is not always easy to define explicit guidelines. Properties such as anterio-posterior gradients, reactivity and asymmetries are well known, but categorizing them as normal, abnormal, or the degree of abnormality, if applicable, is less trivial. Simpler features such as the alpha rhythm frequency and diffused slow-wave activity are better defined, and as shown in Table 3.3, it becomes easier to find agreement in descriptions. Azuma et al. (2003) showed how inter-rater reliability was improved by having three reviewers agree to use the same guidelines (Azuma et al., 2003). It is also shown that consistent terminology can improve clarity and enhance sharing capabilities between clinicians, as well as add value to the reporting and querying of data by storing reviews in an organized database (Aurlien et al., 2004).

Our study focusses on quantifying particular key features of the background pattern as a contribution towards improved consistency and inter-rater reliability. Based on the complexity of EEG signals, strict or "narrow" definitions were avoided to prevent a lack of consensus. Instead, an attempt was made to look for simple guidelines that reviewers can follow to improve consistency when discriminating between normal and abnormal behaviour. Five properties were considered as they were the most common in reports related to our study: i) alpha rhythm peak frequency, ii) alpha reactivity, iii) anterio-posterior gradients, iv) asymmetries, and v) the presence or absence of diffuse slow-wave activity. For EEGs obtained under other circumstances, e.g. intensive care or emergency units, other properties may have more relevance (Cloostermans et al., 2011; Trevathan, 2006; Kurtz et al., 2009).

Previous quantitative analysis studies typically focussed on specialized features to point out a disease or abnormality in the brain (John et al., 1994; Chabot and 
Serfontein, 1996; Hughes and John, 1999; Bares et al., 2012; Prichep et al., 1994; Calzada-Reyes et al., 2011; Begić et al., 2011; Bjork et al., 2011; Jiang et al., 2011; Mishra et al., 2011). The difference between these and our study, is that we attempt to quantify the same background properties reported by routine visual analysis, and in addition, compare the quantitative output to that of the human reviewer. To our knowledge, this has not been done before. Our quantitative algorithms made use of the proposed guidelines and required little or no input from the reviewer to perform its analysis. The outcome was compared to reports from visual interpretation, and as shown in Table 3.3, the agreement between computer-generated features and visual interpretation ranged from slight to substantial. Possible reasons for differences between visual and quantitative analysis can be: i) a too simplistic nature of the proposed guidelines; ii) inconsistencies in visual interpretation; iii) artifacts that interfere with automated analysis; or iv) the quantitative features may lack full support of the guidelines. Also, the thresholds used to categorize the quantitative features were not optimized, and are merely given to indicate how diagnostic classification can be implemented. With ongoing work, more examples of abnormal background patterns will be collected and further optimization will take place.

This study is a first step, only. We are well aware that the background pattern contains more features than the five discussed in the current contribution, e.g. beta activity, mu- and theta-rhythms, lambda waves or physiological transients such as wicket waves. In addition, we quantify features from adult patients during wakefulness. Therefore, the present computer analysis is far from complete. However, its main purpose is to assist in the visual analysis of the EEG, without an attempt to be a replacement of the human EEG reader. The three examples in Section 3.3.6 show how the current system already has a practical use in clinical settings, and with an ongoing study we will focus on improving it further.

Given that the evaluation criteria remains constant, automated analysis can reduce inter-rater reliability if used by the reviewer. As also discussed by Anderson and Doolittle (2010), a reviewer will be less likely to trust an algorithm if he does not understand how its values are calculated. It is desirable to keep the logic of the methods clear and transparent. The presented algorithms were designed with a simple and intuitive approach in mind. Some of them were not capable of accurately quantifying an intended property, and improvements are therefore needed to further optimise the system. A common dataset is also needed to construct a gold standard for evaluating new features. As this dataset develops, the guidelines of defining the various properties should also improve. 
In summary, current EEG analysis techniques can be improved by supplying reviewers with quantitative measures to assist in the review. In order to increase interrater reliability, a consensus is needed to describe EEG properties and to characterize the severity of abnormalities. Five simple guidelines are proposed to describe the EEG background pattern. A quantitative analysis toolkit is developed to describe these properties, and examples show the value added assistance that it can have during a review.

\section{Appendix: Quantitative features}

\subsubsection{Power spectrum}

Let $V(c, t)$ be a matrix representing a single EEG with $c=\{1 . . N\}$ channels over time $t$. First, the entire EEG is split into smaller segments of 5 seconds each:

$$
W\left(c, j, t_{s}\right): j \in[1 . . M], t_{s} \in[0 . .5],
$$

with $j$ referring to each segment and $t_{s}$ to the time in each segment. Using the Welch transform with a 2 second window, zero padded to 2048 sample points and an overlap of $50 \%$, the power spectrum of each segment for each channel is calculated. Let this be represented by

$$
P(c, j, f), c \in[1 . . N], j \in[1 . . M], f \in\left[f_{\min }, f_{\max }\right] .
$$

Each entry in $P(c, j, f)$ contains a discrete Fourier coefficient for channel $c$, segment $j$, and at frequency $f$. The frequency resolution is $0.122 \mathrm{~Hz}$.

\subsubsection{Estimation of the alpha rhythm}

A technique described in (Lodder and van Putten, 2011) was used to find estimates of the alpha rhythm peak frequency in the EEGs. For a full description, refer to the original text.

This technique is based on finding the dominant frequency components between 3-18 Hz in localized segments of the EEG. The dominent frequencies are clustered together, and those most persistent during the eyes closed states are considered to represent the alpha rhythm. Given that the alpha rhythm is most prominent over the occipital region, channels $O 1$ and $O 2$ are used with a common reference montage to estimate the peak frequencies. First, the log spectrum of each segment 
is calculated:

$$
P_{\log }(f)=\log [P(c, j, f)], c \in\{O 1, O 2\}, j \in \boldsymbol{J}_{E C},
$$

where $\boldsymbol{J}_{E C}$ represents the indices of all segments in the eyes closed state. Then, a curve consisting of two peak components and the power-law background spectrum is fitted to $P_{\log }$ :

$$
\begin{aligned}
P_{\log }(f) \approx P_{\mathrm{fit}}(f) & =P_{\mathrm{pk} 1}(f)+P_{\mathrm{pk} 2}(f)+P_{\mathrm{bg}}(f) \\
P_{\mathrm{pk} 1}(f) & =A_{1} \exp \left(\frac{-\left(f-f_{1}\right)^{2}}{\Delta_{1}^{2}}\right), \\
P_{\mathrm{pk} 2}(f) & =A_{2} \exp \left(\frac{-\left(f-f_{2}\right)^{2}}{\Delta_{2}^{2}}\right), \\
P_{\mathrm{bg}}(f) & =B-C \log (f) .
\end{aligned}
$$

The peak parameters are used to form clusters based on frequency similarity, and by taking the average of the largest cluster, the alpha rhythm frequency is estimated accordingly.

\subsubsection{Anterio-posterior gradient}

Using a Laplacian montage for $P(c, j, f)$, the mean power in the alpha band (8$12 \mathrm{~Hz}$ ) is calculated as

$$
P_{\text {alpha }}(c, j)=\operatorname{mean}_{f=\{8 . .12\} H z}[P(c, j, f)],
$$

Segments from the eyes closed state are used to quantify the anterior-posterior gradient. A mean spectrum from these segments are found using

$$
P_{E C}(c)=\operatorname{mean}_{j \in \boldsymbol{J}_{E C}}\left[P_{\text {alpha }}(c, j)\right]
$$

with $\boldsymbol{J}_{E C}$ representing the indices of all segments in the eyes closed state. Using the following channels for the anterior and posterior regions respectively:

$$
\begin{aligned}
& \boldsymbol{C}_{\mathrm{ant}}=\{F p 1, F p 2, F 7, F 8, F 3, F z, F 4\}, \\
& \boldsymbol{C}_{\mathrm{pos}}=\{T 5, T 6, P 3, P 4, P z, O 1, O 2\},
\end{aligned}
$$


the mean alpha power over each region is calculated as

$$
\begin{aligned}
P_{\mathrm{ant}} & =\operatorname{mean}_{c \in \boldsymbol{C}_{\mathrm{ant}}}\left[P_{\mathrm{EC}}(c)\right], \text { and } \\
P_{\mathrm{pos}} & =\operatorname{mean}_{c \in \boldsymbol{C}_{\mathrm{pos}}}\left[P_{\mathrm{EC}}(c)\right] .
\end{aligned}
$$

Following from this, a normalized ratio for the anterior-to-posterior power is found:

$$
Q_{\mathrm{APG}}=\frac{P_{\mathrm{ant}}}{P_{\mathrm{ant}}+P_{\mathrm{pos}}}
$$

\subsubsection{Asymmetries}

Left-right symmetry is calculated by comparing spectral power between the two hemispheres. To increase robustness, power ratios are first calculated in individual segments and then averaged together. Using the channel pairs

$$
\begin{aligned}
C_{\{\mathrm{L}, \mathrm{R}\}}=\{ & \{F p 1, F p 2\},\{F 7, F 8\},\{F 3, F 4\},\{T 3, T 4\}, \\
& \{C 3, C 4\},\{T 5, T 6\},\{P 3, P 4\},\{O 1, O 2\}\},
\end{aligned}
$$

a left-right ratio for each pair is calculated:

$$
L R\left(c_{\{L, R\}}, j, f\right)=\frac{P\left(c_{\{\mathrm{R}\}}, j, f\right)-P\left(c_{\{\mathrm{L}\}}, j, f\right)}{P\left(c_{\{\mathrm{R}\}}, j, f\right)+P\left(c_{\{\mathrm{L}\}}, j, f\right)}, c_{\{L, R\}} \in C_{\{\mathrm{L}, \mathrm{R}\}} .
$$

By calculating the mean of all segments:

$$
L R_{\mathrm{AVG}}\left(c_{\{L, R\}}, f\right)=\left|\operatorname{mean}_{j=\{1 . . M\}}\left[L R\left(c_{\{L, R\}}, j, f\right)\right]\right|,
$$

and averaging over $f=\{0.5 . .12\} \mathrm{Hz}$, a single value is obtained to quantify the symmetry for each channel pair:

$$
Q_{\mathrm{ASYM}}\left(c_{\{L, R\}}\right)=\operatorname{mean}_{f=\{0.5 . .12\} \mathrm{Hz}}\left[L R_{\mathrm{AVG}}\left(c_{\{L, R\}}, f\right)\right] .
$$

\subsubsection{Diffused slowing}

Diffuse slow-wave activity is detected by finding a ratio in spectral power from a low $\{2 . .8\} \mathrm{Hz}$ band to a wider $\{2 . .25\} \mathrm{Hz}$ band. Using the common reference 
montage to calculate $P(c, j, f)$, the following channels are used to find the ratio:

$$
\begin{array}{r}
C_{\mathrm{SLOWING}}=\{F 7, F 8, F 3, F 4, F z, T 3, T 4, T 5, T 6, \\
C 3, C 4, C z, P 3, P 4, P z, O 1, O 2\} .
\end{array}
$$

Using only segments from the eyes closed state, the mean spectrum over all channels are found:

$$
P_{E C}(f)=\operatorname{mean}_{c \in \boldsymbol{C}_{\text {SLOWING }}}\left[\operatorname{mean}_{j \in \boldsymbol{J}_{E C}}[P(c, j, f)]\right],
$$

with $\boldsymbol{J}_{E C}$ referring to the indices of all segments in the eyes closed state. The total power in the low and wide spectral bands are calculated:

$$
\begin{aligned}
P_{\text {low }} & =\sum_{f=2 H z}^{8 H z} P_{E C}(f), \\
P_{\text {wide }} & =\sum_{f=2 H z}^{25 H z} P_{E C}(f),
\end{aligned}
$$

and from these the slowing-ratio is found:

$$
Q_{\text {SLOWING }}=P_{\text {low }} / P_{\text {wide }} .
$$





\section{Chapter 4}

Computer-assisted interpretation of the EEG background pattern: A clinical evaluation

Shaun S. Lodder Jessica Askamp Michel J. A. M. van Putten 


\begin{abstract}
Interpretation of the background pattern in routine EEG recordings is an important part of clinical reviews. We evaluated the feasibility of an automated system to assist reviewers with evaluating the EEG background pattern. Quantitative EEG methods were used to describe the following five background properties: posterior dominant rhythm frequency and reactivity, anterior-posterior gradients, presence of diffuse slow-wave activity and asymmetry. Software running the quantitative methods was sent to nine experienced electroencephalographers together with 45 routine EEG recordings and automatically generated reports. Participants were asked to first review the EEGs by visual analysis and then to correct mistakes made by the system. Corrected reports were returned for comparison. Using a goldstandard derived from the consensus of reviewer reports, inter-rater agreement was calculated for all reviewers and for automated interpretation. Automated interpretation together with most participants showed high (kappa >0.6) agreement with the gold standard. In some cases, automated analysis showed higher agreement with the gold standard than participants. When asked, all participants considered computer-assisted interpretation useful for routine reviews. Automated interpretation methods showed clinical feasibility and was well received by all reviewers.
\end{abstract}

Significance: Computer-assisted interpretation of the EEG background pattern can bring consistency to reports and improve efficiency and inter-rater agreement. 


\subsection{Introduction}

Scalp EEG is used in a wide range of clinical settings to obtain a non-invasive measurement of cortical brain activity. Having a higher temporal resolution and being more affordable, portable and widely available than $\mathrm{FMRI}$ and MEG, its uses range from diagnostics and monitoring for outpatient recordings, to continuous monitoring in the ICU. The recordings are typically analyzed by visual inspection of the signals in their raw form, and apart from being a time consuming and error prone task which may lead to missed events, this can also result in high inter- and intra-rater variability depending on the level of experience and degree of concentration of the reviewer (Anderson and Wisneski, 2008).

An important part of EEG reviews is the analysis of the background pattern. For routine outpatient recordings this plays an important part in epilepsy diagnostics (Wilson and Emerson, 2002; Halford, 2009), clinical psychiatry (Coburn et al., 2006) and the diagnosis of neurodegenerative diseases (Petit et al., 2004; Babiloni et al., 2011; Moretti et al., 2012). Also, continuous monitoring of the background pattern in the ICU can alert medical staff of sudden changes that require immediate intervention (Friedman and Hirsch, 2010; Cloostermans et al., 2011; Foreman and Claassen, 2012), thereby changing its role from passive to an active tool for improving the outcomes of the critically ill.

Quantitative EEG analysis (QEEG) and structured reports have been proposed to lessen the burden of visual reviews and to add more consistency during reporting (Aurlien et al., 2004; van Putten, 2008; Halford, 2009; Anderson and Doolittle, 2010; Beniczky et al., 2013). Standard guidelines for writing EEG reports state that objective observations of the EEG properties should be made first, followed by the conclusions drawn from the reviewer based on these observations. ${ }^{1}$ As such, quantitative analysis is well suited during the first phase, i.e. by assisting in the objective description of all background properties in a consistent manner. Given that other factors such as medication and patient history are not known or taken into consideration by quantitative analysis, conclusions drawn after initial observations should be left to the reviewer.

As shown in (Azuma et al., 2003), inter-rater agreement for describing EEG observations can be improved if reviewers agree to follow a clear set of guidelines in reporting their findings. These guidelines should be obtained from the general

\footnotetext{
${ }^{1}$ See Guideline 7 provided by the American Clinical Neurophysiology Society, J Clin Neurophysiol. 2006 Apr;23(2):118-21.
} 
consensus of experienced electroencephalographers themselves. In recent work reported in (Beniczky et al., 2013), the authors show on how such a set of guidelines and definitions are being constructed as part of a pan-European project with the goal of providing more consistency and structure for the reporting in clinical EEG reviews (Beniczky et al., 2013; Aurlien et al., 2004, 2007).

Many types of quantitative EEG features have been proposed to describe specific properties in the EEG. These include statistical measures such as variance, kurtosis and skewness (Scherg et al., 2012; Stevenson et al., 2013), non-linear energy operators (Mukhopadhyay and Ray, 1998), small-world networks and functional connectivity (Stam et al., 2007; Bullmore and Sporns, 2009), synchrony (Lachaux et al., 1999; van Putten, 2003), entropy (Stam, 2005; Kannathal et al., 2005), power ratios (Kurtz et al., 2009; Cloostermans et al., 2011), bi-spectral index (Sigl and Chamoun, 1994), and left-right symmetry (van Putten et al., 2004). Despite the variety of complex features available, relatively simple measures can be used to describe many of the background properties of an EEG. Example features are the presence or absence of certain rhythmic components, power ratios between delta-, theta-, alpha- and beta-bands, and the power distribution over the scalp. The importance of each background property will vary based on the reason for recording, but in general, a description of the background pattern is of significant importance for any review.

In a previous study, we described an automated system based on quantitative features that can be used to assist with the interpretation of the EEG background pattern (Lodder and van Putten, 2013). Based on the methods described in (van Putten, 2008), this system uses quantitative analysis to estimate properties for the posterior dominant rhythm, reactivity, anterior-posterior gradients, presence of diffuse slow wave activity, and symmetry, and then determines if these properties are abnormal or fall within the normal range. Together with constructing an automated report based on the outcome of these features, the system also shows the quantitative properties to the user in a simple and intuitive manner.

The results in (Lodder and van Putten, 2013) showed that quantitative features can be used to find accurate measures of the common EEG background properties. It was however also shown that regardless of good predictions, the system was not reliable enough to match all outcomes from the diagnostic reports based on visual analysis. It can therefore only serve to assist during a review and not replace visual analysis. A reason for lower agreement with the diagnostic reports could also have been related to a lack of consistent reporting from visual analysis that lead to higher inter- and intra-rater variability, and a follow-up goal was therefore 
to measure inter-rater agreement in standard reports for these properties.

Although some background properties have more relevance than others depending on the clinical setting and reason for recording, all of these considered form part of the common properties described in most routine EEG reports. The goal of this study was to evaluate this system in routine outpatient EEG recordings by sending it to a group of qualified and experienced electroencephalographers, and to determine if there is any additional benefit in computer assisted interpretation together with conventional EEG reviews. Apart from evaluating the accuracy of the automated system, we also wanted to measure the inter-rater agreement between reviewers, and in addition to this obtain feedback from participants about their experience and perceived importance of using quantitative features for future reviews. Fast and accurate interpretation of the background pattern by means of computer assisted analysis can save time for reviewers, reduce the costs of analysis, bring consistency and completeness to EEG reports, reduce inter-rater variability, and allow for both experienced and less experienced electroencephalographers to benefit from additional visualizations of the EEG by presenting it in a simpler and more intuitive manner.

\subsection{Methods}

\subsubsection{Subjects and data}

The dataset used for this study consisted of 45 anonymized routine scalp EEGs, each 20-30 min in length. Regarding ethical approval for using the EEG data in this study, according to Dutch law, researchers do not need to consult a medical ethical committee if patient data has been obtained as part of routine patient care. Furthermore, patient consent is not needed for additional use of these data for further scientific research if the data has been anonymized. These statements have been confirmed by our medical ethical committee. All recordings were obtained from the Medisch Spectrum Twente hospital in the Netherlands. Original diagnostic reports were used to find example EEGs in such a way that both normal and abnormal occurrences were available for each background property. Apart from ensuring that both normal and abnormal occurrences exist, the dataset was chosen randomly with recording dates ranging over 7 years. Our selection was unbiased towards the number of artifacts each recording contained, and subject ages ranged from 10 to 88 years (mean 53.2). Patients were awake during the recording and a standard 2030 min protocol was used, which included hyperventilation and photic stimulation. 


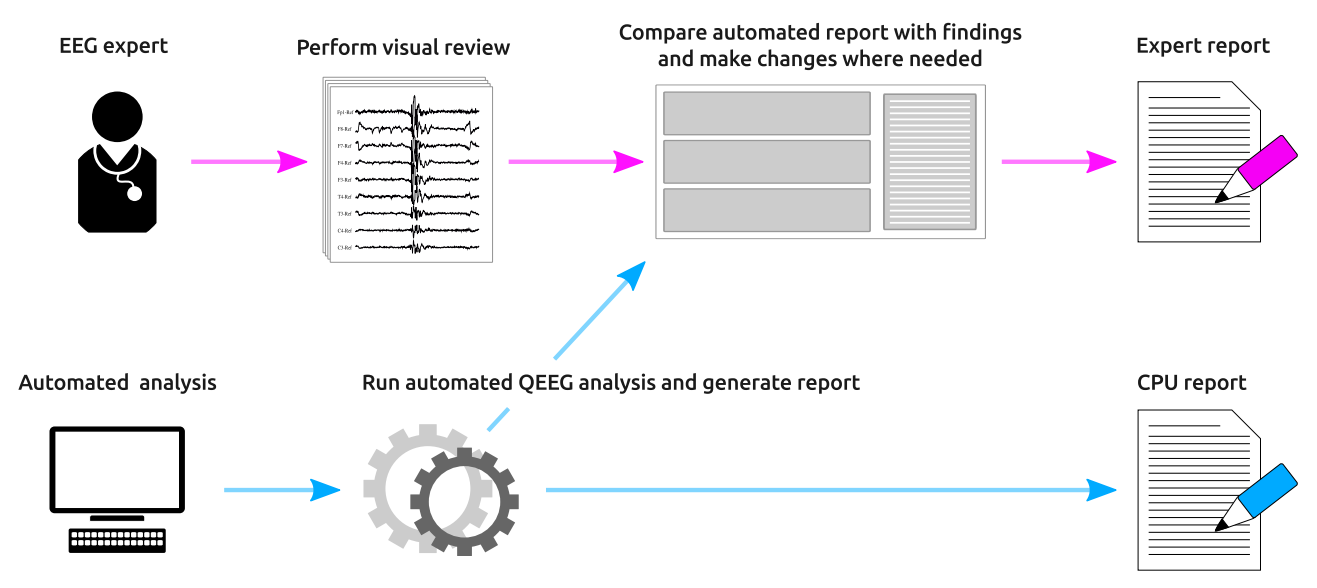

Fig. 4.1: Outline of the study: For each of the 45 recordings, participants were asked to open the EEG in the conventional way (Fig. 4.2) and review five background properties by visual inspection. After this, they were asked to open a new window showing a summary of the quantitative features (Fig. 4.3), and to correct the mistakes made in a report generated by automated interpretation.

None of the recordings were sleep-deprived EEGs. The EEGs were recorded at a sample rate of either 250 or $256 \mathrm{~Hz}$ with the Brainlab EEG system, and $\mathrm{Ag}-\mathrm{AgCl}$ electrode caps were used with electrodes placed according to the 10-20 system. Impedances were kept below $5 \mathrm{k} \Omega$ to reduce polarization effects.

\subsubsection{Automated interpretation of the background pattern}

As a first step, quantitative features were calculated for each EEG in the dataset. A brief outline on the calculation of each quantitative feature is provided in the Appendix, and a detailed description can be found in (Lodder and van Putten, 2013). Five background properties were considered, and based on the threshold values provided in the Appendix, an automated description of each property was obtained. The five background properties were: $i$ ) the posterior dominant rhythm frequency and ii) its reactivity, iii) anterior-posterior gradients, iv) asymmetries, and $v$ ) the presence or absence of diffuse slow-wave activity. In the case of asymmetry, the system also determined the affected regions in which asymmetries appeared. Available options for this were the left and right frontal, central, temporal, parietal, and occipital regions. The calculated findings were stored in diagnostic reports that were later presented to the reviewers for verification. To make the outcomes compatible with visual reviews and easier to compare, set categories were defined in the reports for the outcomes of each property. These categories are shown in Table 4.1. 


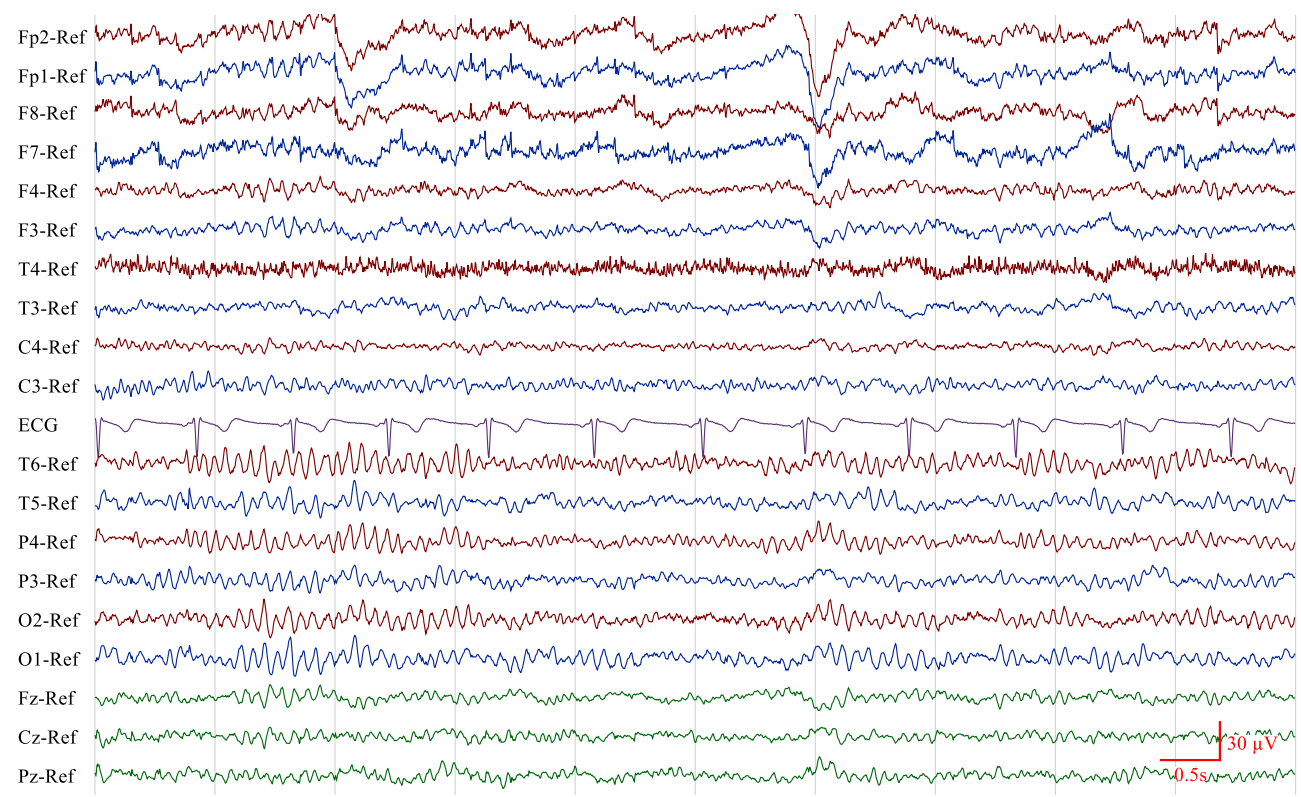

Fig. 4.2: Before evaluating the automated reports and correcting mistakes made by quantitative analysis, participants were asked to review the EEG conventionally by visual inspection of the recording in its raw form.

\subsubsection{Visual inspection and confirmation}

To compare automated reporting with visual analysis, the same dataset was sent to ten certified and experienced electroencephalographers across multiple centers within the Netherlands, together with a set of instructions and examples on how to interpret the displayed quantitative features. Fig. 4.1 shows an outline of the protocol followed by each participant. For each of the 45 recordings, participants were asked to first open the EEG as usual (i.e. ten second pages of raw time series data) as shown in Fig. 4.2, scroll through the recording, and review the five background properties by visual inspection. After this, they were asked to open a new window in the software application showing the same recording, but this time using quantitative features instead of the raw time series data. In this new window was also the automatically generated report of the EEG background properties. An example of this display is shown in Fig. 4.3. The participants were then asked to verify the outcome of the automatically generated reports with their interpretation from visual inspection and make changes to it where automated analysis was wrong. To edit the reports, a user interface with predefined checkboxes for the outcome of each 


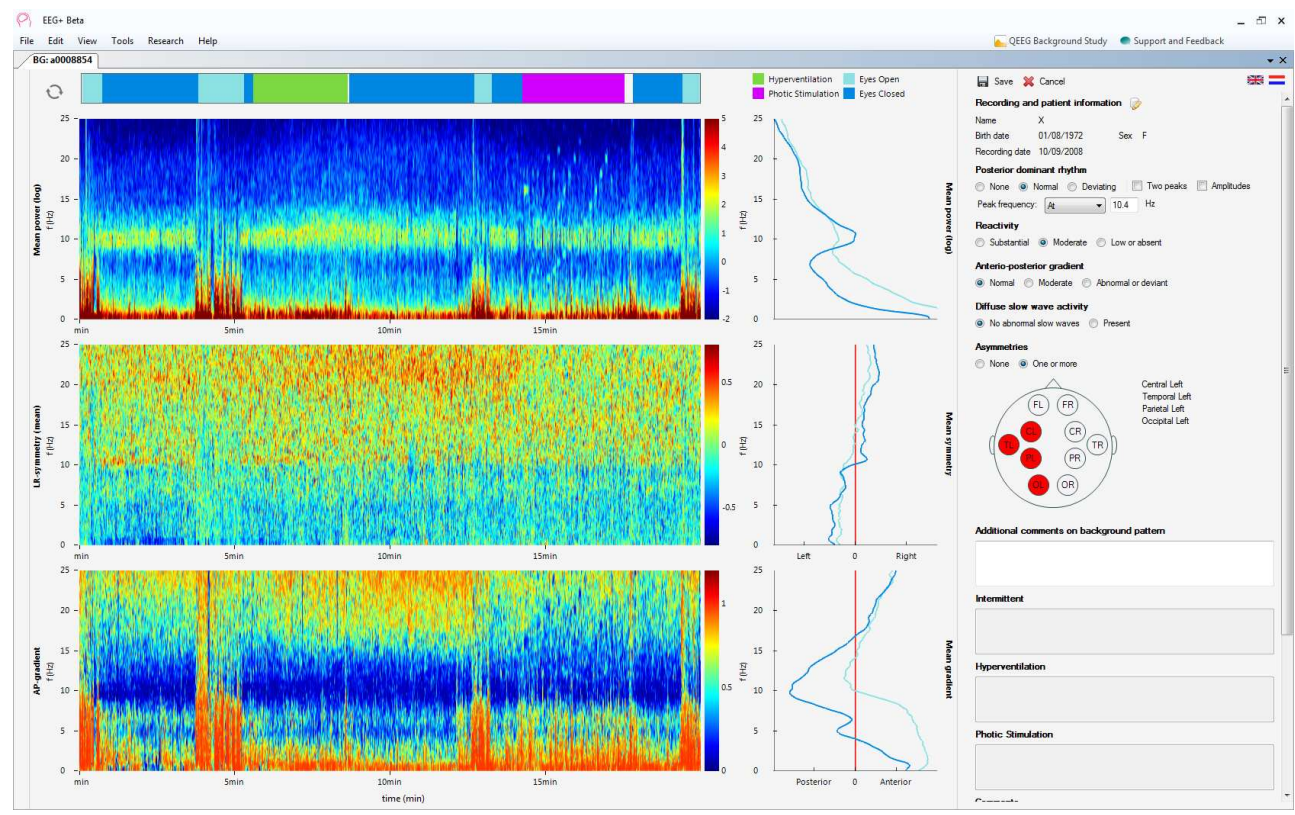

Fig. 4.3: After visually reviewing the EEG in its raw form, participants were shown a quantitative EEG display which summarizes the entire recording into a single window. Using the report provided in this window (right), participants were asked to correct the automated interpretation where needed and afterwards upload the corrected reports for comparison.

property was provided, as shown on the right of Fig 4.3. Possible outcomes for each property are summarized in Table 4.1. Together with this, participants also had the opportunity to compare their findings from visual analysis with the quantitative features displayed on the left side of the quantitative analysis screen (Fig. 4.3). After updating the reports and confirming that all findings were correct, the reports were uploaded to our server and later analyzed for comparison.

\subsubsection{User experience}

Given that the system would also require general acceptance by reviewers to become clinically relevant, an attempt was made to measure the user experience and willingness of participants to include assisted interpretation into their routine reviewing procedure. To obtain a quantitative measure for this, participants were asked five questions after evaluating all the EEGs. These questions, together with the respondent averages, are shown in Table 4.2. The questions were focused on obtaining information about the reviewers' previous experience with quantitative 
EEG analysis, its ease of use, and their perceived importance of quantitative EEG analysis in future reviews.

\subsection{Results}

To determine the feasibility of computer-assisted reviewing, the first important step was to determine the accuracy and robustness of the quantitative analysis methods. This was done by calculating the inter-rater agreement between the computer-generated reports and a gold standard, which was obtained by taking the most agreed upon outcome for each property in each of the recordings as given by the ten participants. Using the gold standard for comparison, the inter-rater agreement was calculated for both the automatically generated reports and for individual participants that served as a benchmark. Inter-rater agreements were calculated using Fleiss' Kappa measure, and the results of these comparisons are shown in Table 4.4. Although no universally excepted rule exists for interpreting Kappa values, a popular guideline is provided by (Landis and Koch, 1977) and will be used here accordingly (see Table 4.3 for reference). The first ten rows in Table 4.4 show the inter-rater agreement between each participant and the gold standard for each of the background properties. Compared with the gold standard, nearly all participants showed substantial $(0.61-0.80)$ and almost perfect $(>0.81)$ agreement for all properties apart from the presence and location of asymmetries. For the presence of asymmetries, four participants showed moderate agreement $(0.41-0.60)$ and the remaining six substantial agreement or higher. In reporting the location of the asymmetries, two participants showed moderate agreement and the remaining participants all substantial or almost perfect agreement. One participant did not specify the asymmetry regions. The last row of Table 4.4 shows the interrater agreement between the the gold standard and the automatically generated reports as obtained by automated analysis. Here we see that for the PDR, reactivity, anterior-posterior gradient and slowing, the system obtained almost perfect agreement with the gold standard. For asymmetries and asymmetry regions, the system obtained almost perfect and substantial agreement respectively. Also seen is that the system showed higher agreement with the gold standard than some reviewers.

Considering the questionnaire that participants were asked to complete at the end of the study, two said that they have never used quantitative analysis before or during a routine or long-term review, whereas the others have all used quantitative 
Table 4.1: To make outcomes comparable with visual reviews, set categories were defined in the reports for the outcomes of each property.

Posterior dominant rhythm (PDR)

None Normal Deviating

PDR frequency

$f(\mathrm{~Hz})$

Reactivity

Substantial Moderate Low or absent

AP-Gradient

Normal Moderate Deviating

Abnormal diffuse slow waves

Yes present None

Asymmetries

None One or more

Affected asymmetry region

Left Right Both None

measures mostly on an occasional, regular or routine basis. Quantitative analysis also appears to be used with same rate in long-term recordings as in routine EEGs. Regarding the question asking participants if they found the automatically generated reports useful during their review, two said only rarely and the remaining eight occasionally. Half of the participants also said that with the use of quantitative features they expect to spend less time to perform a review, whereas the other half said that they expected to spend the same amount of time as with a conventional review. None of the participants expected to spend more time. Lastly, asking the participants if they would find it useful to have quantitative analysis methods assist them during their daily interpretation of both routine and long-term EEGs, all of them responded with yes.

\subsection{Discussion}

This study investigated the clinical relevance of computer assisted interpretation of the EEG background pattern. Our specific goal was to test the quantitative anal- 
Table 4.2: Five questions were asked to each participant after all EEGs were reviewed.

1. Before this study, how often have you used any form of quantitative analysis methods to help you with

(a) Routine EEG reviews?

Never (11\%) Rarely (0\%) Occasionally (44\%) Often (22\%) Always (22\%)

(b) Long-term EEG reviews?

Never (11\%) Rarely (11\%) Occasionally (33\%) Often (33\%) Always (11\%)

2. How often did the quantitative analysis figures or generated reports alter your conclusions based on visual interpretation of the EEG (eg. picked up a missed asymmetry or different degree of reactivity)?

Never (0\%) Rarely (22\%) Occasionally (78\%) Often (0\%) Always (0\%)

3. Did you find the automatically generated reports useful during your review?

Not at all (0\%) Rarely (0\%) Occasionally (44\%) Often (33\%) Most of the time (22\%)

4. In your opinion, do you think that compared to the standard way of reviewing EEGs, the added quantitative features will make you spend more time, less time, or the same amount of time to perform a review?

$$
\text { More }(0 \%) \quad \text { Same }(56 \%) \quad \text { Less }(44 \%)
$$

5. Will you find it useful to have quantitative analysis methods assist you in every day interpretation of:

(a) Routine EEGs

$$
\text { No }(0 \%) \quad \text { Yes }(100 \%)
$$

(b) Long-term EEGs

$$
\text { No }(0 \%) \quad \text { Yes }(100 \%)
$$

ysis methods described in (Lodder and van Putten, 2013) and to determine if they bring added value to conventional reviewing procedures. To do this, an EEG dataset consisting of 45 routine scalp EEGs was given to both the automated interpretation system described in (Lodder and van Putten, 2013) and to ten experienced electroencephalographers for review. Using a gold standard derived from the reviewer reports, the inter-rater agreement was calculated for each participant and for the automatically generated reports by themselves.

From Table 4.4 we see that there was high inter-rater agreement between all reviewers for all of the background properties described, and only once was the 
Table 4.3: Interpretation of Kappa values as suggested by (Landis and Koch, 1977)

\begin{tabular}{ll}
\hline$\kappa$ & Interpretation \\
$<0$ & Poor agreement \\
$0.01-0.20$ & Slight agreement \\
$0.21-0.40$ & Fair agreement \\
$0.41-0.60$ & Moderate agreement \\
$0.61-0.80$ & Substantial agreement \\
$0.81-1.00$ & Almost perfect agreement \\
\hline
\end{tabular}

Table 4.4: Inter-rater agreement between the gold standard and reviewers $(\mathrm{R}\{\mathrm{i}\})$, and the gold standard and automated analysis (CPU) respectively. The gold standard was based on the most agreed upon outcome for each property using reports from the participants, and the inter-rater agreement was measured using Fleiss' Kappa.

\begin{tabular}{lllllll}
\hline Reviewer & PDR & Reactivity & AP-Gradient & Slowing & Asymmetries & Asymmetry regions \\
R1 & 0.87 & 0.91 & 0.85 & 1.00 & 0.72 & 0.77 \\
R2 & 0.87 & 0.66 & 0.58 & 0.81 & 0.72 & 0.71 \\
R3 & 0.93 & 0.91 & 0.95 & 1.00 & 0.70 & - \\
R4 & 0.63 & 0.72 & 0.69 & 0.80 & 0.78 & 0.86 \\
R5 & 0.67 & 0.76 & 0.86 & 0.74 & 0.80 & 0.74 \\
R6 & 0.87 & 0.69 & 0.63 & 0.85 & 0.59 & 0.59 \\
R7 & 0.77 & 0.34 & 0.65 & 0.78 & 0.61 & 0.61 \\
R8 & 0.94 & 0.91 & 0.90 & 1.00 & 0.76 & 0.54 \\
R9 & 0.88 & 0.60 & 0.82 & 1.00 & 0.54 & 0.62 \\
CPU & 0.93 & 0.91 & 0.95 & 1.00 & 0.70 & 0.77 \\
\hline
\end{tabular}

inter-rater agreement less than moderate for any of the properties (reactivity for reviewer R7). Although the quantitative algorithms followed a specific set of guidelines to determine the difference between normal and abnormal properties as described in (Lodder and van Putten, 2013), participants were not explicitly asked to do the same. The reason for this is that in some cases, these guidelines may be too simplistic in nature to make accurate interpretations. We therefore allowed the reviewers to make interpretations based on their own experience, and for future use we intend to combine the guidelines used in (Lodder and van Putten, 2013) with the proposed guidelines given by (Beniczky et al., 2013), and will encourage users to use this instead to ensure conformity and standardization of EEG reports. Following the same set of guidelines may also help to improve the inter-rater agreement, as demonstrated in (Azuma et al., 2003).

An important observation in Table 4.4 is that the automatically generated reports had higher agreement with the gold standard than many of the reviewer reports. 
This is an interesting finding, but should be interpreted carefully given that a bias was added by asking participants to correct the generated reports instead of letting them fill out the entire report by themselves. As a partial compensation to avoid this bias, participants were not shown the pre-calculated reports until after they drew their own conclusions based on visual inspection of the entire recording, as described in the methods and shown in the experiment outline in Fig.4.1. Due to the inclusion of a possible bias however, we cannot conclude that automated analysis is more in agreement with the gold standard than some reviewers, although it does point to the fact that automated analysis is more consistent with interpretation than some reviewers. To make a fair comparison between visual analysis and automated interpretation, a similar study will have to be done where participants are asked to fill out reports without receiving input from quantitative analysis. Our aim however is not to replace the reviewer but instead to assist him, and it was therefore chosen to perform the study in this way in order to also receive feedback from participants based on their experience of using computer assisted interpretation.

This study made use of partially structured reports to describe the EEG background properties. Although provision was not made for all the background features, an additional text box was available for reviewers to add additional comments or to expand their descriptions on specific properties if they wished to do so. Together with other improvements, more categorized properties can be added to provide a fully structured report, as shown by for example (Aurlien et al., 1999, 2004). Structured reports make it easier for reviewers to follow general guidelines in describing EEG properties, and helps them to perform their review of each property in a consistent manner, without leaving anything out.

Although the five questions asked to the participants at the end of the study were only general in nature, our objective with this was to measure the overall acceptance of computer assisted methods together a conventional EEG review. Automated interpretation of the general properties associated with the EEG background pattern has been suggested before (Nakamura et al., 1992, 2002; Zhang et al., 2011), but up to now is still not widely accepted for routine clinical use. Based on the received answers from the questionnaire however, we do see that many participants make use of quantitative EEG measures in some way or another. Other quantitative systems are typically aimed at describing one or two specific properties in the EEG, for example markers pointing to neurodegenerative diseases (Gudmundsson et al., 2007; Snaedal et al., 2012; Petit et al., 2004) or psychiatric disorders (Coburn et al., 2006), and trends showing burst-suppression rates or seizures in long-term ICU monitor- 
ing (Cloostermans et al., 2011; Foreman and Claassen, 2012). Regarding the automatically generated reports for describing the general background properties of the EEG, most participants considered it to be useful during their review.

One of the important goals of this study was to try and improve the overall interrater agreement in describing the EEG background pattern. When participants were asked if the generated reports altered their initial conclusions based on visual analysis alone, most of them indicated that it had done so occasionally. This shows that although quantitative analysis may also make mistakes, it becomes useful as an assistant during reviews and helps to improve reviewer consistency and intra-rater reliability. Therefore, given that none of the reviewers will expect to take longer to perform their reviews by adding computer-assisted analysis, this approach may benefit the final outcome of an EEG review without reducing the reviewer efficiency. Given that the recording and review of EEGs are also one of the highest costs involved with neurological visits apart from MRI and EMG (Burke et al., 2013; Strzelczyk et al., 2012), any improvement in efficiency should lead to a significant reduction in overall healthcare costs. All participants also indicated that they would find it useful to have quantitative analysis methods assist them with every day interpretation of routine and long-term EEGs, showing a very positive sign for the general acceptance of computer assisted reviewing.

The system presented here is of course far from perfect and many improvements can still be made to provide a better and more efficient user experience. For some features, in particular the detection of asymmetries, more detailed and also more accurate interpretations from the automated system are needed to improve the inter-rater agreement. Also, the interpretation of additional properties such as mu and beta rhythms, lambda waves, and response to hyperventilation and photic stimulation for example, are needed to to provide a complete description of the EEG background pattern. Although there was no selection bias in the chosen dataset regarding artifacts, it can be assumed that the system will become less accurate if too many artifacts exist. However, given our sample size of $45 \mathrm{EEGs}$ that also included the normal amount of artifacts expected from a routine recording, the system appears to be fairly robust. In the case where artifacts would severely affect the performance of the system, they will clearly be seen on the summarized review screen (left side) as shown in Fig. 4.3. The reviewer should then be advised not to trust the outcome of the automated review.

It is important to keep in mind that, as also stated in (Anderson and Doolittle, 2010), quantitative features and automated systems should remain transpar- 
ent where possible and care should be taken not to over-complicate algorithms and thereby lose the confidence of the reviewer. Regarding the visualization of the quantitative features as shown in Fig. 4.3, some participants have also commented on non-intuitive parts of the display, leading to them making less use of it. Further work is therefore needed to improve the quantitative displays and to find more intuitive methods to clearly show and summarize the EEG background properties.

In summary, a successful and accurate implementation of computer assisted interpretation of the EEG background pattern can assist reviewers in their daily routine of reviewing EEGs. Together with the structured reports obtained by this system, this will bring more consistency to reviewing and further improve the interrater agreement. Simple and intuitive ways of showing quantitative features can also summarize and present the entire recording on a single display and thereby bring added benefits to both experienced and inexperienced reviewers alike, and in addition help to reduce the reviewing time significantly.

\section{Acknowledgments}

The authors would like to extend their gratitude to all participants that took part in reviewing the EEGs; Dr. Geert Brekelmans, Prof. Oebele F. Brouwer, Prof. J. Gert van Dijk, Dr. Jeannette Hofmeijer, Dr. Frans S. S. Leijten, Dr. Jan Meulstee, Dr. Jaco Pasman, Dr. Robjan Schimsheimer, and Dr. Selma C. Tromp. We also thank them for all their suggestions and insightful comments.

\section{Appendix: Quantitative background features}

Five background properties were evaluated and interpreted with quantitative measures in this study. They were: i) the posterior dominant rhythm frequency and ii) its reactivity, iii) anterior-posterior gradients, iv) asymmetries, and $v$ ) the presence or absence of diffuse slow-wave activity. A brief outline of the various properties are provided below, and for more details, the reader is referred to (Lodder and van Putten, 2013).

For the posterior dominant rhythm, a peak frequency was calculated over the occipital region by dividing each EEG into segments of five seconds each and using a curve-fitting approach to find the dominant peaks in the eyes-closed segments. Afterwards, the peak estimates of all segments were clustered together to determine the dominant frequency components, and from this an estimate of the posterior 
dominant peak frequency was obtained. A full description of this technique is described in (Lodder and van Putten, 2011).

After obtaining the peak dominant frequency, the reactivity of the posterior dominant rhythm was calculated by measuring the mean occipital power in a $0.5 \mathrm{~Hz}$ frequency band around that frequency when the eyes are open $P_{E O}$ and and when the eyes are closed $P_{E C}$ :

$$
Q_{R E A C}=1-\frac{P_{E O}}{P_{E C}} .
$$

The reactivity was classified as substantial when $Q_{R E A C}>0.5$, moderate for $0.1<$ $Q_{R E A C}<0.5$, and low or absent if $Q_{R E A C}<0.1$.

Anterior posterior gradients were measured by calculating the mean power over the anterior $P_{a n t}$ and posterior regions $P_{\text {pos }}$ of the scalp, and finding a normalized value that quantifies the contribution of $P_{\text {ant }}$ to the total power:

$$
Q_{A P G}=\frac{P_{a n t}}{P_{a n t}+P_{a n t}} .
$$

The alpha power gradient was considered within normal range if $Q_{A P G}$ was lower than 0.4 , moderately differentiated between 0.4 and 0.6 , and abnormal or deviant for values above 0.6 .

In a similar manner, asymmetries were found by comparing power differences between matching left- and right electrode pairs:

Lastly, the presence of diffuse slow-wave activity was calculated by comparing the power in lower theta and delta bands $(2-8 \mathrm{~Hz}) P_{l o w}$ to a wider spectral band ranging from $2-25 \mathrm{~Hz} P_{\text {wide }}$ :

$$
Q_{S L O W I N G}=\frac{P_{\text {low }}}{P_{\text {wide }}} .
$$

Diffuse slow-wave activity was considered to be present if $Q_{S L O W I N G}>0.6$, i.e., less than $40 \%$ of the power was above $8 \mathrm{~Hz}$. 
Part II

Inter-ictal epileptiform

discharges 



\section{Chapter 5}

\section{Inter-Ictal Spike Detection using Smart Templates}

Shaun S. Lodder Jessica Askamp

Michel J. A. M. van Putten 


\begin{abstract}
Visual analysis of EEG is time consuming and suffers from inter-observer variability. Assisted automated analysis helps by summarizing key aspects for the reviewer and providing consistent feedback. Our objective is to design an accurate and robust system for the detection of inter-ictal epileptiform discharges (IEDs) in scalp EEG. IED Templates are extracted from the raw data of an EEG training set. By construction, the templates are given the ability to learn by searching for other IEDs within the training set using a time-shifted correlation. True and false detections are remembered and classifiers are trained for improving future predictions. During detection, trained templates search for IEDs in the new EEG. Overlapping detections from all templates are grouped and form one IED. Certainty values are added based on the reliability of the templates involved. For evaluation, 2160 templates were used on an evaluation dataset of 15 continuous recordings containing 241 IEDs $(0.79 / \mathrm{min})$. Sensitivities up to $0.99(7.24 \mathrm{fp} / \mathrm{min})$ were reached. To reduce false detections, higher certainty thresholds led to a mean sensitivity of 0.90 with $2.36 \mathrm{fp} / \mathrm{min}$. By using many templates, this technique is less vulnerable to variations in spike morphology. A certainty value for each detection allows the system to present findings in a more efficient manner and simplifies the review process.
\end{abstract}

Significance: Automated spike detection can assist in visual interpretation of the EEG which may lead to faster review times. 


\subsection{Introduction}

Epilepsy is estimated to affect around 50 million people worldwide. With a high temporal resolution that can capture inter-ictal epileptiform discharges (IEDs), EEGs play an important role in the diagnosis of epilepsy. A major drawback however, is that reviewing them is time-consuming. Also, the diagnostic yield is low, partially due to the relative short duration of routine EEG recordings, and due to this, multiple routine recordings are typically required before signs of inter-ictal epileptiform activity are found (Doppelbauer et al., 1993). Given that reviewers have different levels of training and experience, a reasonably high inter-observer reliability is also known to exist (Azuma et al., 2003; Abend et al., 2011).

Longer recordings have shown to increase the chances of finding inter-ictal epileptiform activity (Faulkner et al., 2012; Agbenu et al., 2012; Leach et al., 2006; Friedman and Hirsch, 2009). In some cases this can lead to fewer follow-up recordings and an overall increase in diagnostic efficiency. Unfortunately, longer recordings also result in more time required for visual analysis, a burden that is best avoided. Computerized assistance with the detection of IEDs can lessen the burden of visual analysis, and as an added benefit, ensure more consistency between reviews that will lower inter-rater variability. A large number of detection algorithms have already been proposed over the last four decades, ranging from template matching and parametric methods, to mimetic analysis, spectral analysis and artificial neural networks (see (Wilson and Emerson, 2002) and (Halford, 2009)). A review by Halford (2009) shows that some techniques have achieved very promising results, but due to a lack of a common dataset, comparisons between methods are still hard to make.

One of the main obstacles in IED detection is not only to find the inter-ictal events, but also to minimize the number of false detections per minute. If this number is too large, a reviewer will still be required to inspect most of the data and automated detection will be of no use. Most methods for this reason use a multi-step process to first detect possible events, and then discard artifacts and non-epileptiform events with additional rules from the set of potential candidates. Although difficult to compare without a common dataset, a good benchmark for the feasibility of spike detection methods in practice are their sensitivities and false detection rates. Low sensitivities show that reviewers can miss important IEDs if any are present, and as mentioned, high false detection rates make evaluating the output of the algorithm more time consuming than a visual review itself. Although 
selectivity measures also provide useful information about the algorithm, it does not take into account the number of IEDs per minute in the dataset, which is critical when comparing methods from different datasets. Recent methods report average sensitivities of 0.90 (Nonclercq et al., 2012), 0.65 (De Lucia et al., 2008), 0.82 (Argoud et al., 2006), 0.93 (Subasi, 2006), 0.78 (Halford et al., 2012), 0.69 (Ji et al., 2011a), and 0.92 (Van Hese et al., 2008), and average false detections per minute of 6 (De Lucia et al., 2008), 13.4 (Argoud et al., 2006), 0.92 (Indiradevi et al., 2008), and 0.09 (Ji et al., 2011a). In most studies that report high sensitivities, a high false detection rate is present if given, otherwise data epochs are used instead of continuous EEG, or the number of false detections are not reported (Nonclercq et al., 2012; Subasi, 2006; Halford et al., 2012; Indiradevi et al., 2008; Van Hese et al., 2008). On the other hand, studies with low false detection rates show lower sensitivities. This shows the trade off for all methods between choosing high sensitivities with more false detections, or lower sensitivities with fewer false detections.

Regardless of the many algorithms available, few implementations have made it to clinical practice. With the aim of achieving a practical and reliable system for IED detection, we introduce an automated detection method based on template matching to find IEDs in EEG recordings. The method is unique in the sense that it uses a large database of templates extracted from a training set of example EEGs. Unlike other template matching algorithms that only rely on a single or a small number of templates to search for inter-ictal events, our system can match more variations in spike morphology and is less vulnerable to variability. In addition, the algorithm is designed to incorporate experience into the templates from past classifications during training, which can be extended even further so that they can gain additional experience with repeated use. The presented method is intended to assist instead of replace visual inspection, with the aim of significantly reducing review time for clinicians and neurologists.

\subsection{Methods}

\subsubsection{Subjects and data}

An EEG dataset for this study was obtained from the department of Clinical Neurophysiology at the Medisch Spectrum Twente (MST) in the Netherlands. Recordings were made with a standard 20-30 minute protocol using a Brainlab EEG system and standard $\mathrm{Ag}-\mathrm{AgCl}$ electrode caps placed according to the international 10-20 guidelines. Impedances were kept below $5 \mathrm{k} \Omega$ to reduce polarization effects. A 
sample rate of either $250 \mathrm{~Hz}$ or $256 \mathrm{~Hz}$ was used for each recording. Afterwards it was band-pass filtered between $0.5-30 \mathrm{~Hz}$, and downsampled to $100 \mathrm{~Hz}$ to increase efficiency of the algorithm. IEDs were marked and reviewed by experienced electroencephalographers on the channels where they were clearly visible (MvP and JA). Each IED consisted of one of the following patterns: (i) spike, (ii) sharp wave, (iii) spike and slow wave, (iv) sharp wave and slow wave (v) slow wave with absent or very small preceding spike, or (vi) polyspike and slow wave. IEDs were marked in three montages: common reference, bi-polar and source.

The dataset consisted of 23 records with 723 IEDs in total and a combined recording length of $481 \mathrm{~min}$. The patient group consisted of 15 males and 8 females with ages ranging from 4 to 52 (mean $23.2 \pm 17.1$ ) years. Thirteen subjects were diagnosed with generalized epilepsies ( 4 absence, 1 juvenile myoclonic, 8 idiopathic), and the remaining ten with focal epilepsies ( 5 temporal lobe, 1 rolandic, 4 other). The dataset was split into two parts, one for template collection and training, and the other for an evaluation set. Eight EEGs were used for training and fifteen for testing. The training set contained 482 marked IEDs with a total recording length of 175 min. The testing set contained 241 marked IEDs with a total recording length of $306 \mathrm{~min}$.

\subsubsection{Preprocessing}

Independent component analysis was used to reduce the influence of eye blink artifacts. After calculating the independent components with the FastICA algorithm ${ }^{1}$, each component was compared to the electrooculogram (EOG) channel recorded with the EEG. If a component showed a substantial correlation with the EOG channel $(>0.5)$, it was removed by setting all its values to zero. The remaining components were projected back to their channel space by applying the inverse transform.

\subsubsection{Method outline}

The presented method uses a database of templates resembling typical waveforms of epileptiform activity, and finds matches between the templates and interictal events in the EEG. The main concepts that make this method different from others are the following: (i) a substantially larger set of templates is used, (ii) the templates are designed to have experience by remembering past classifications and

\footnotetext{
${ }^{1}$ Available at http://research.ics.aalto.fi/ica/fastica/
} 
using this to improve future predictions, and (iii) an agreement system is used to combine detected events from multiple templates and thus lower false detections. The sections that follow give a detailed outline of the various parts of the method, which can be divided into template collection, learning, and detection. An illustrative summary of the template collection and learning phase is shown in Fig. 1, and one for the detection phase in Fig. 2.

\subsubsection{Template collection}

Starting with a blank database, templates are added using a training dataset. For each EEG in the training set, a collection of epochs is extracted at the locations where IEDs were marked by the reviewers. Each epoch represents a template and is added to the database. Three montages are used during collection: common reference, bi-polar, and source. Templates are extracted per channel, i.e. if an IED is marked over two channels in the common reference montage and one channel in the bi-polar montage, three templates will be created. Single spikes or sharp waves were not included as templates, due to a high degree of false detections. An example of extracted templates is shown in Fig. 3. Note that they also have differences in magnitude (amplitudes were not normalized, but used 'as recorded').

\subsubsection{Learning}

After extracting the templates, their ability to find and discriminate between other IEDs from the same or other recordings and non-epileptiform activity is determined. This is done by finding a time-shifted correlation between each template and all EEG channels for all EEGs in the training set with the same montage. Due to the computational cost involved, this part of the algorithm was implemented with parallel processing. Locations are found where the templates have correlations above 0.85 , and the underlying EEG segment is extracted to calculate additional properties to further determine the relationship between them and the template by which they were detected. For this, let a template consisting of $N$ samples ( $N$ differs per template) be defined as $S_{\text {tem }}(n): n \in[1 . . N]$, and each segment it finds as $S_{\text {seg }}(n): n \in[1 . . N]$, as illustrated in Fig. 4. In addition, let the preceding $N$ samples of each segment (same length as segment) be defined as $S_{\text {prec }}(n): n \in[1 . . N]$ and the variance of the channel on which the segment is located as $\sigma_{c h}^{2}$. Then, the 
following properties are calculated:

$$
\begin{aligned}
f_{C R R} & =\operatorname{corr}\left(S_{\text {tem }}, S_{\text {seg }}\right), \\
f_{\text {DCRR }} & =\operatorname{corr}\left(S_{\text {tem }}^{\prime}, S_{\text {seg }}^{\prime}\right), \\
f_{\text {MAD }} & =\frac{1}{N} \sum_{n}^{N}\left\|S_{\text {tem }}(n)-S_{\text {seg }}(n)\right\|, \\
f_{\text {VRCHAN }} & =\frac{\sigma_{\text {seg }}^{2}}{\sigma_{\text {seg }}^{2}+\sigma_{\text {ch }}^{2}}, \\
f_{\text {VRTEM }} & =\frac{\sigma_{\text {seg }}^{2}}{\sigma_{\text {seg }}^{2}+\sigma_{\text {tem }}^{2}}, \text { and } \\
f_{\text {VRPREC }} & =\frac{\sigma_{\text {seg }}^{2}}{\sigma_{\text {seg }}^{2}+\sigma_{\text {prec }}^{2}},
\end{aligned}
$$

where

$$
\begin{aligned}
\sigma_{\text {seg }}^{2} & =\operatorname{var}\left(S_{\text {seg }}\right), \\
\sigma_{\text {tem }}^{2} & =\operatorname{var}\left(S_{\text {tem }}\right), \text { and } \\
\sigma_{\text {prec }}^{2} & =\operatorname{var}\left(S_{\text {prec }}\right) .
\end{aligned}
$$

In addition to having a high correlation with the template $\left(f_{C R R} \geq 0.85\right)$, these properties are used to determine the similarity between each detected segment and its template. Property $f_{D C R R}$ measures the correlation between the derivatives of $S_{\text {seg }}$ and $S_{\text {tem }}, f_{\text {MAD }}$ finds the mean amplitude difference between $S_{\text {seg }}$ and $S_{\text {tem }}$, and $f_{V R C H A N}, f_{V R T E M}$ and $f_{V R P R E C}$ calculates the variance ratio between $\sigma_{\text {seg }}^{2}$ and the sum of $\sigma_{\text {seg }}^{2}$ with $\sigma_{c h}^{2}, \sigma_{\text {tem }}^{2}$ and $\sigma_{\text {prec }}^{2}$ respectively.

Using these properties, the location of each segment is converted to a "nomination" of an IED event if it satisfies both of the following conditions: (i) $f_{\text {MAD }}<$ $20 \mu \mathrm{V}$, and (ii) $f_{V R C H A N}>0.75$ or $f_{V R P R E C}>0.67$. Given that the correlation between two segments is scale invariant, the first requirement ensures that their magnitude is similar. The second requirement is based on the assumption that, for scalp EEG, the amplitude changes within an IED segment will be significantly larger than its preceding segment and the channel variance. Threshold values were chosen arbitrarily. Given that the true locations of IEDs are known in the training set, each 

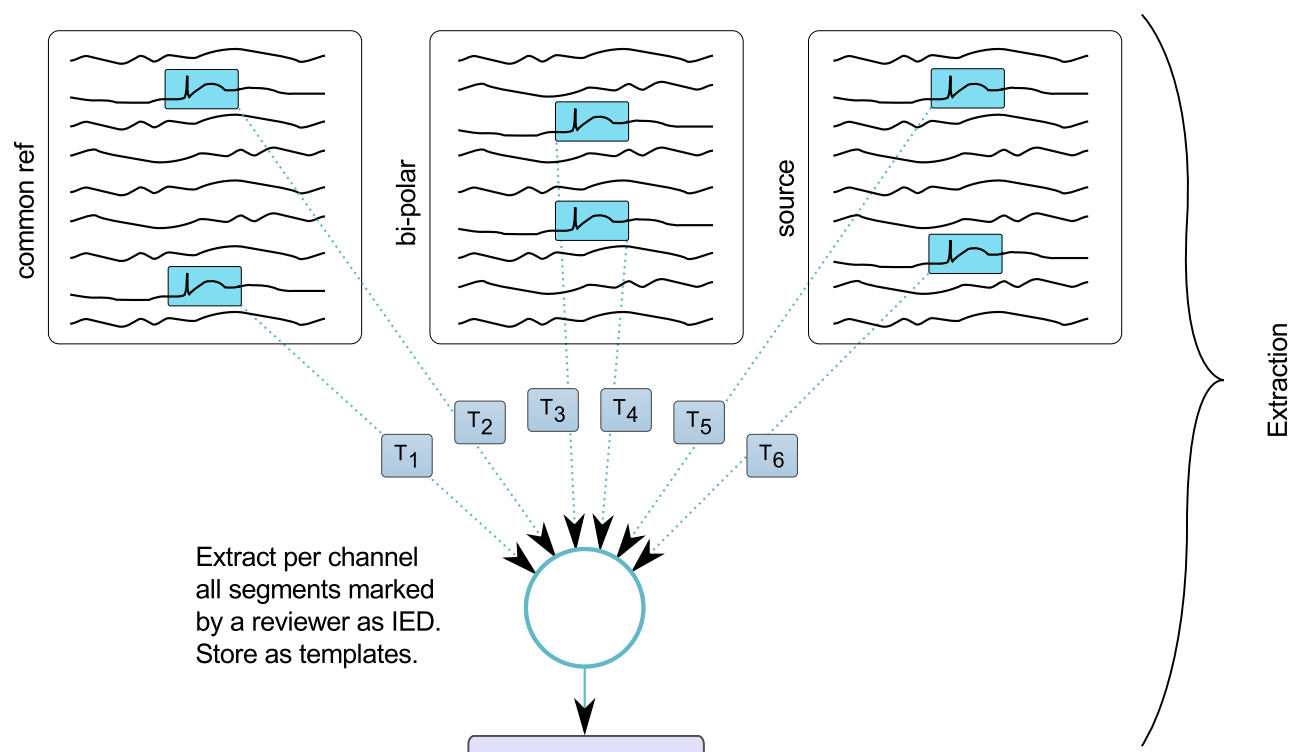

Extract per channel all segments marked by a reviewer as IED. Store as templates.

\section{\begin{tabular}{lllllll}
$T_{2}$ & $T_{3}$ & $T_{4}$ & $T_{5}$ & \\
\hdashline & & & & $T_{6}$
\end{tabular}}

을

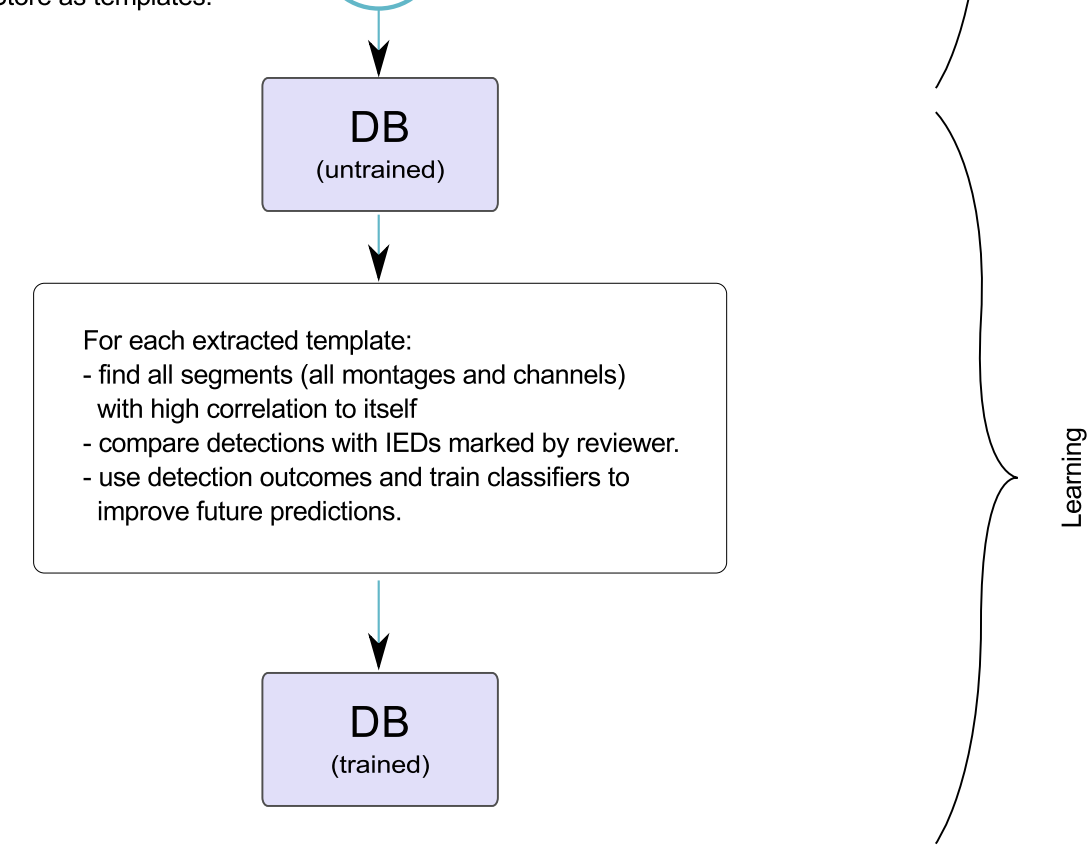

Fig. 1: Outline of the template extraction and learning steps. After the templates are trained, each of them contributes to the detection by nominating possible IEDs.

nomination is either marked as correct or as a false detection, depending on if any of its samples overlap in time with a marked IED. At this point, the false detection 


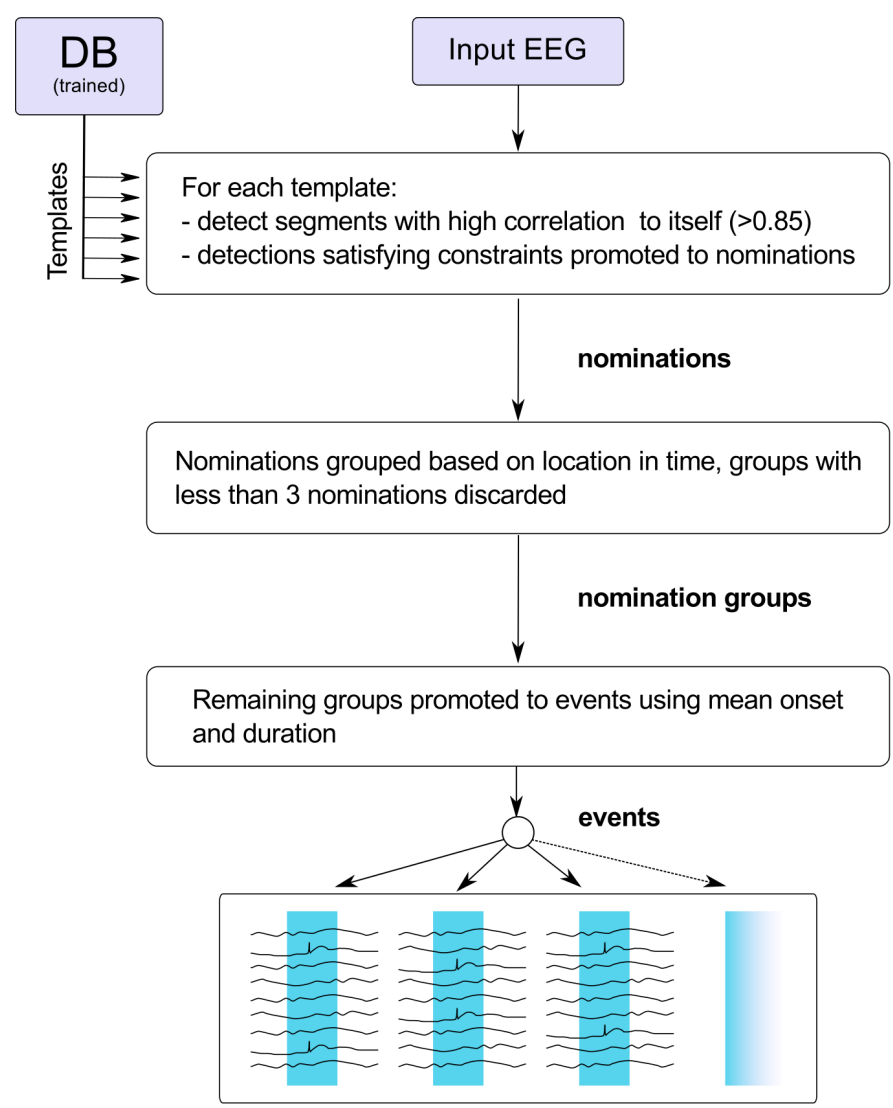

Fig. 2: Outline of the detection method. Trained templates detect and nominate possible IEDs. The system combines these nominations and identifies IEDs based the the mutual agreement of multiple templates.

rate of a template can be calculated:

$$
F D R_{\text {tem }}=\frac{\text { false detections }}{\text { true detections }+ \text { false detections }} .
$$

Using only the criteria above to nominate events as IEDs lead to many false detections. Therefore, a linear support vector machine (SVM) is trained using the nominations in the training set to give the template additional experience in discriminating between true IEDs and false predictions. Vectors consisting of $\left\{f_{C R R}, f_{D C R R}, f_{\text {VRTEM }}\right\}$ are used as input. By having an SVM trained on past classifications, the template can now find events with high correlation to itself and predict if it is in fact an 

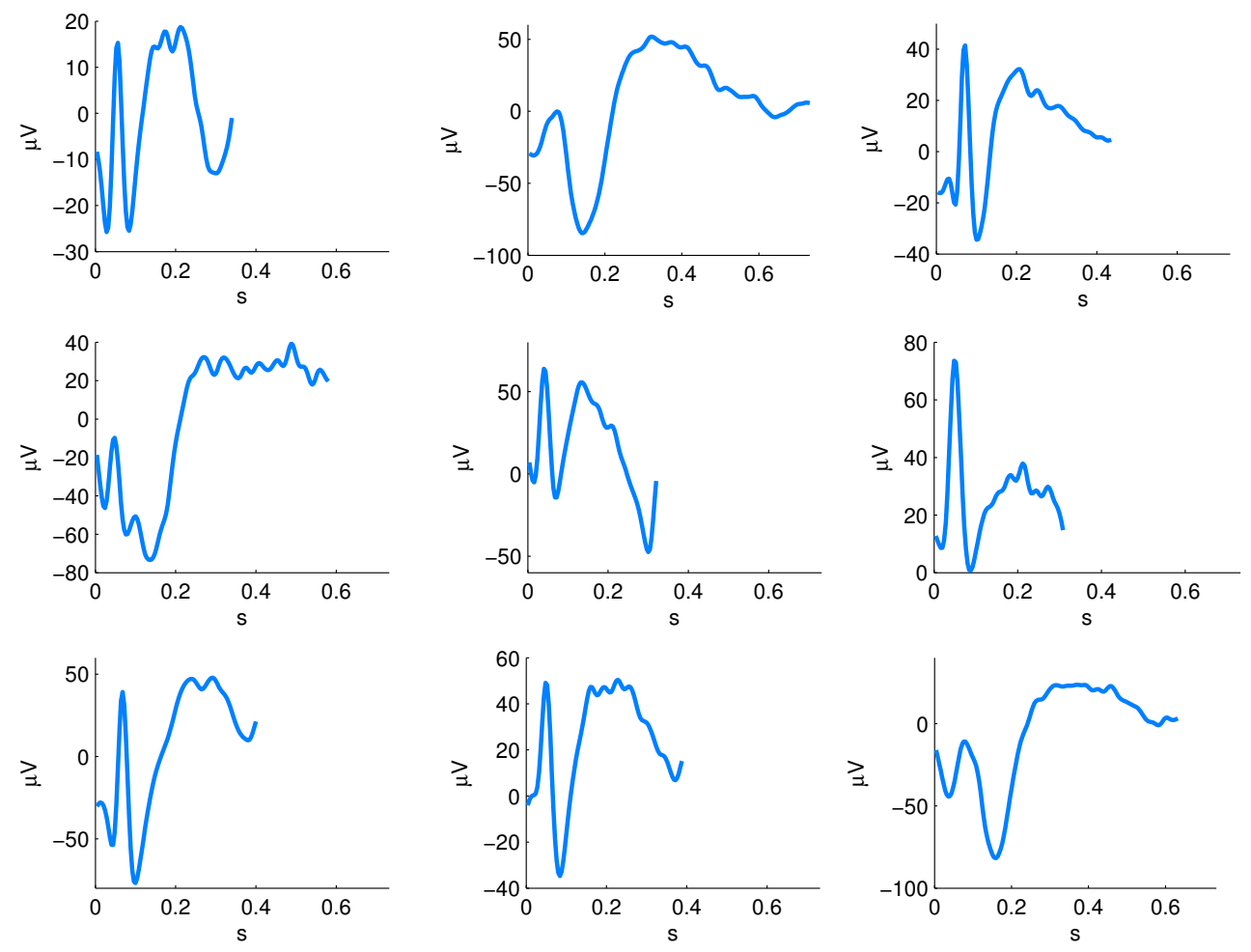

Fig. 3: Nine templates taken from the trained database consisting of 2160 templates. Template lengths are allowed to differ and amplitudes are preserved.

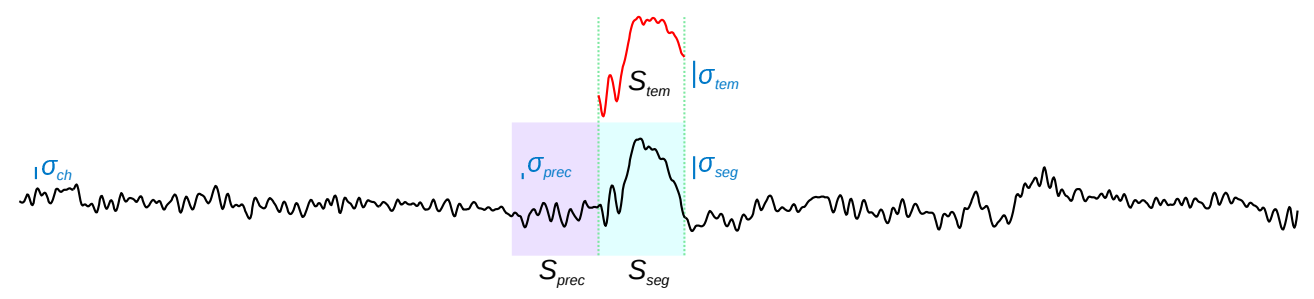

Fig. 4: Template features: To find matching events, templates use correlation coefficients, amplitude differences and variance ratios between a template and its detected segment. Variance ratios add temporal context to a detected event by comparing it to its surrounding activity.

epileptiform event or not. After a template's SVM is trained, its reliability $\left(R_{t e m}\right)$ is calculated as the accuracy of re-classifying its own training vectors. The reliability reflects a templates ability to separate IEDs from non-epileptiform events, and is used as a weight factor during detection. 
After all training steps are complete, templates that do not comply with the following conditions are discarded: (i) Templates with zero detections, (ii) templates with only false detections, (iii) templates with a small number of correct detections and no false detections. The last condition avoids templates that only find themselves in the training set and which have no use in finding other IEDs.

\subsubsection{Detection}

For detection, a similar approach is followed as during learning: For each template in the database, a time-shifted correlation is calculated on each EEG channel. Locations with correlations above 0.85 are nominated as IEDs if they also satisfy the conditions: (i) $f_{\text {MAD }}<20 \mu \mathrm{V}$ and (ii) $f_{V R C H A N}>0.75$ or $f_{V R P R E C}>0.67$, and using each template's own SVM, a prediction is made if the event is in fact an IED or only a similar looking artifact. False predictions are discarded and the remaining events are converted to nominations and added to a global pool. Nominations are stored with the following information about themselves: $(a)$ its onset and duration, (b) the channel on which it was found, (c) $f_{C R R}$, and (d) $R_{t e m}$.

Next, nominations are grouped together if they reside on the same channel and overlap with more than $75 \%$. Groups with fewer than 3 nominations are considered unreliable and are discarded. Each of the remaining groups is considered a detected event, and the onset and duration is taken from the mean of the nominations in the group. The event is also assigned a detection certainty, which is done by calculating the mean product of each nomination's correlation and template reliability:

$$
\text { certainty }=\frac{1}{M} \sum_{m}^{M} R_{t e m}(m) f_{C R R}(m),
$$

with $M$ the number of nominations in that group. Lastly, events from individual channels are merged into one if they overlap in time, and once again the mean onset, duration and certainty is used to describe the event.

\subsection{Results}

A total of 2632 templates were collected during training, of which 472 were discarded for not passing the constraints described in Section 5.2.5. To gain some insight on the behavior of the remaining 2160 templates, histograms of FDR tem and $R_{\text {tem }}$ are provided in Fig. 5.5(a) and Fig. 5.5(b) respectively. The mean false detec- 


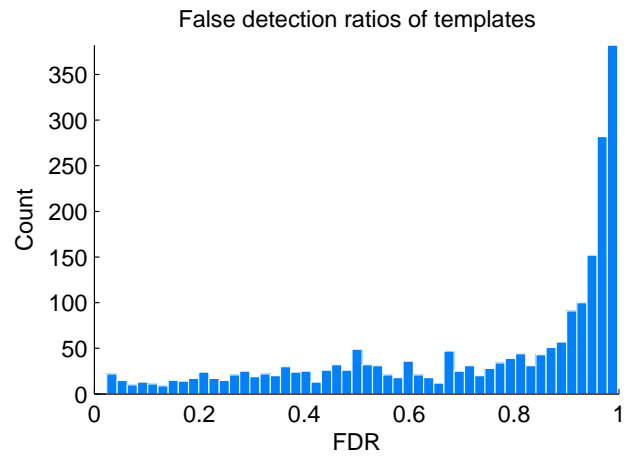

(a)

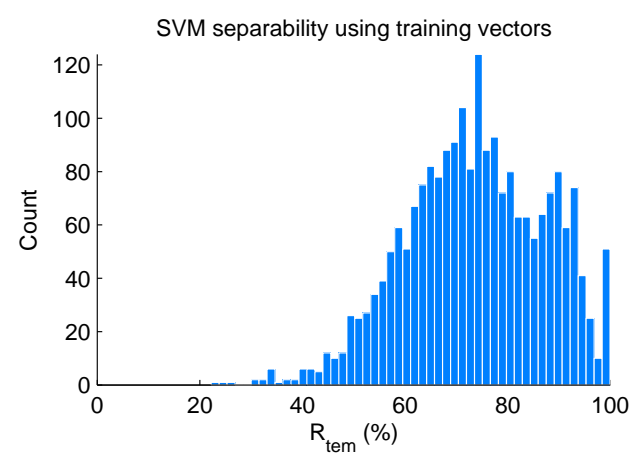

(b)

Fig. 5: Histograms combining individual template statistics: (a) false detection ratios; and (b) $R_{\text {tem }}$ : the ability of each template's SVM to separate its training vectors. The two histograms show that by themselves the templates have high false detection rates and in many cases poor separability between IEDs and non-IED events. The power of the system lies in combining the information and using a network effect to allow accurate predictions.

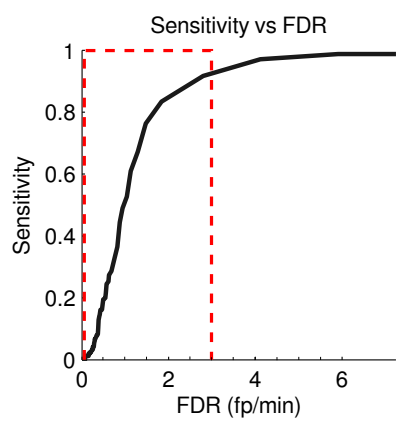

(a)

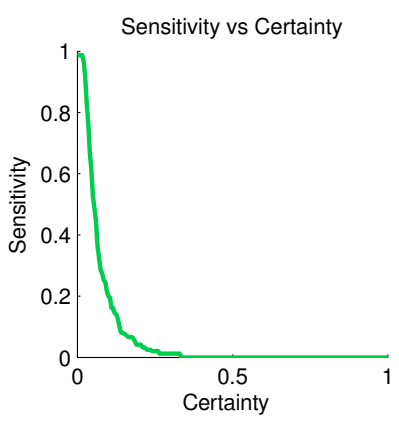

(b)

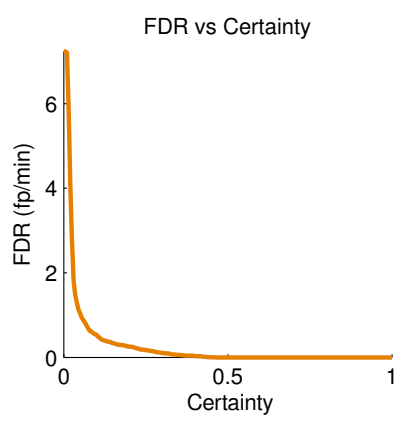

(c)

Fig. 6: Evaluation of the described method on a test set of 15 EEGs: (a) an ROC curve shows how the sensitivity of detecting IEDs can be improved at the price of more false detections. The dashed rectangle gives an approximate range of false detection rates that we consider as more feasible for practical use; (b) higher confidence values reduce the sensitivity, (c) but also lowers the false detection rate.

tion rate before training SVMs is 0.74 and the mean template reliability after SVM training $73.8 \%$. The two histograms show that the templates by themselves have high false detection rates and suboptimal accuracies in finding IEDs. Fig. 5.5(b) shows however how the use of SVMs and additional properties can help reduce the number of false detections and improve accuracy. Fig. 5.6(a) gives an ROC curve from all detected events in the test EEGs, and it shows that although low accuracies 
are achieved at template level, the combined information from individual templates lead to reliable detections on a global level. As seen in Fig. 6, the certainty threshold can be adjusted to either maximize the sensitivity or minimize the false detection rate. Figures 5.6(b) and 5.6(c) respectively show the sensitivity and false detection rate as a function of minimum certainty, and Table 1 gives corresponding values to a number of points on the curves in Fig. 6. For all test EEGs combined, a maximum sensitivity of 0.99 is reached at a false detection rate of 7.24 false positives per minute. Note that the IED rate in the evaluation set is only 0.79 IEDs per minute, and so a false detection rate of 7.24 will be unacceptable for practical use. Although far from ideal, we consider a more acceptable false detection rate for reviewing to be below three per minute. The mean sensitivities obtained after adjusting the certainty threshold of each EEG individually is shown in Table 2. The thresholds were chosen in such a way as to limit the false positives per minute to no more than three. After this, a mean sensitivity of 0.90 and mean false detection rate of $2.36 \mathrm{~min}^{-1}$ is achieved. The selection of a threshold cannot be chosen a priori to limit the false detection rate to a known maximum, but this shows that detections with higher certainties are more reliable. In a typical usage scenario, a user may choose to view events with the highest certainties first, and then gradually reduce the threshold if he wishes to see more.

In addition to the detection algorithm, the described method was also implemented into an EEG viewer. Fig. 7 shows how each of the detected events are presented, and using the options provided, the reviewer can decide to either confirm, reject, or indicate doubt concerning each detected event. In such a way, the method can be used to assist during a review by pointing out areas of interest in the EEG and allowing the reviewer to give the final verdict.

\subsection{Discussion}

Automated detection of inter-ictal epileptiform discharges is a crucial step towards improving the efficiency of epilepsy diagnostics and monitoring. Not only does it reduce the review time significantly and make longer recordings feasible, but it also allows for more objective analysis with less inter-observer variability. The presented method uses a large collection of templates to detect inter-ictal epileptiform discharges. It keeps track of past classifications during training in order to provide the templates with additional statistical experience, and the size of the database allows it to contain example spike-and-slow-wave patterns of many mor- 
Table 1: Choosing to maximize the sensitivity or minimize the false detection rate can be done by adjusting the minimum confidence threshold. This allows a reviewer to view the most probable IEDs first, and then to look at additional events if desired. Values below correspond to points on Fig. 6(a)-(c).

\begin{tabular}{lll}
\hline Min confidence & Mean sensitivity & Mean Fp/min \\
0 & 0.99 & 7.24 \\
0.02 & 0.97 & 4.12 \\
0.04 & 0.67 & 1.29 \\
0.06 & 0.44 & 0.87 \\
0.08 & 0.27 & 0.62 \\
0.1 & 0.2 & 0.54 \\
0.12 & 0.15 & 0.41 \\
0.14 & 0.08 & 0.37 \\
0.16 & 0.07 & 0.31 \\
0.18 & 0.06 & 0.29 \\
0.2 & 0.04 & 0.26 \\
0.22 & 0.03 & 0.22 \\
0.24 & 0.02 & 0.19 \\
0.26 & 0.02 & 0.16 \\
\hline
\end{tabular}

Table 2: Mean sensitivities and false detection rates per EEG in the test set using a varying confidence threshold value per EEG and limiting false detections to a maximum of three per minute.

\begin{tabular}{lllll}
\hline EEG & Dur (min) & IEDs & SEN & Fp/min \\
S1 & 16.3 & 13 & 1.00 & 1.59 \\
S2 & 22.5 & 11 & 0.82 & 2.93 \\
S3 & 20.0 & 5 & 1.00 & 1.40 \\
S4 & 20.0 & 36 & 0.97 & 2.60 \\
S5 & 22.3 & 19 & 0.79 & 1.93 \\
S6 & 19.5 & 6 & 1.00 & 2.72 \\
S7 & 20.3 & 79 & 0.97 & 1.82 \\
S8 & 21.2 & 5 & 0.80 & 1.98 \\
S9 & 20.0 & 2 & 1.00 & 2.95 \\
S10 & 20.0 & 12 & 1.00 & 2.00 \\
S11 & 21.5 & 7 & 0.38 & 2.88 \\
S12 & 20.3 & 7 & 0.86 & 2.95 \\
S13 & 20.7 & 19 & 0.95 & 2.08 \\
S14 & 21.0 & 14 & 1.00 & 2.95 \\
S15 & 20.0 & 6 & 1.00 & 2.60 \\
mean & $\mathbf{2 0 . 4}$ & $\mathbf{1 6 . 0 7}$ & $\mathbf{0 . 9 0}$ & $\mathbf{2 . 3 6}$ \\
\hline
\end{tabular}


phologies so that IEDs can be detected across multiple patients and recordings, regardless of whether their inter-ictal patterns look the same. In addition, using the experience of each template, the system can determine its reliability during detection. This approach resembles the way in which humans search for IEDs: by finding waveforms that have properties associated with IEDs, and mentally comparing them to patterns observed in the past. In addition to high correlations between templates and detected EDs, additional features were chosen to add information and improve detection accuracy. Given that correlations are scale independent, the mean amplitude difference $\left(f_{M A D}\right)$ ensures that matching events are on the same scale, and the variance ratios $\left(f_{V R C H A N}, f_{V R T E M}, f_{V R P R E C}\right)$ ensure that IEDs are significantly different from its surrounding activity. By adding these properties, the method is capable of not only using wave morphology, but also temporal context.

To improve accuracy, the system removes uncertainties of single templates by using a network effect to make decisions. In other words, even though individual templates by themselves miss events and make many false detections, the collective value of their votes result in accurate detections. Using this approach, the system is less vulnerable to variability in spike morphology than other methods which rely on single features or a small number of templates. Still, the system is highly dependent on the templates contained within its database. Our results show that mosts IEDs can be detected with the current set of templates and thresholds described. However, the ideal set (and number) of templates to find all common inter-ictal patterns is not known, and it could be that additional templates with more variations in spike and wave morphology will allow the system to match events more accurately, allowing higher thresholds for individual detections, and thereby lowering the overall false detection rate.

A major advantage of this technique is that the system remains dynamic and that more templates can be added if required. By further extending the method and adding a feedback loop during reviews, the database can become adaptive. As an example, missed events can be added as templates if a reviewer finds any IEDs visually during a review. Another example is if template reliabilities change when new EEGs are reviewed and false detections are marked by a reviewer. As currently implemented, templates search for matching IEDs in parallel to each other. Although not strictly neccesary, this speeds up the detection process significantly and makes the system scalable and fast enough for clinical use. Our implementation of the method runs on a standard desktop computer (Intel i7-2600 $3.40 \mathrm{GHz}$ CPU, 4 GB RAM) and requires no additional hardware. 


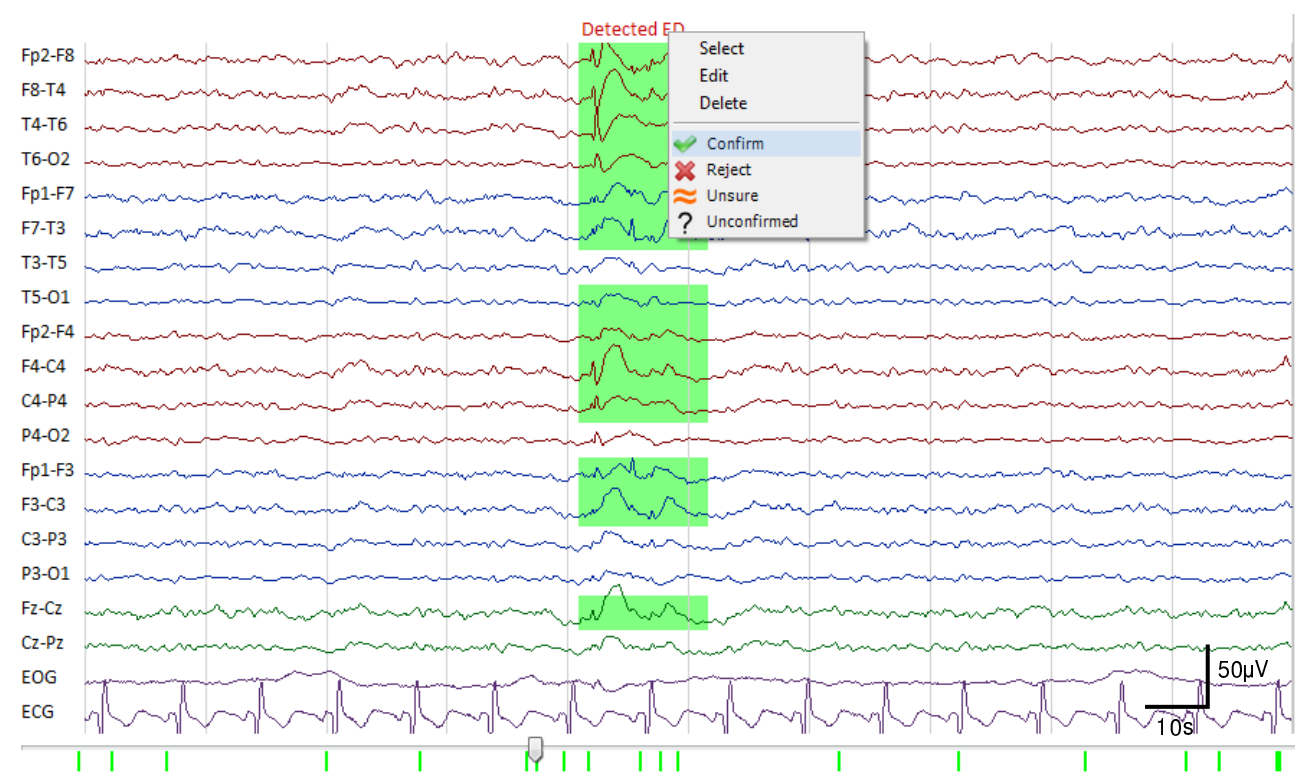

Fig. 7: A detected IED highlighted on top of the EEG, as displayed to the reviewer in a bi-polar montage.

Reviews by Wilson and Emerson (2002) and Halford (2009) summarize most of the reported techniques over the last four decades. In addition to these, newer methods have also been reported (Nonclercq et al., 2009, 2012; Scherg et al., 2012; Ji et al., 2011a,b; Igasaki et al., 2011; Argoud et al., 2006; Nenadic and Burdick, 2005; Wang et al., 2010; Zhou et al., 2012). Comparisons between different methods are hard to make because there is no common dataset currently available. Without a benchmark dataset, reported results rely heavily on the data used. For example, some studies use standard 20 minute recordings to evaluate their methods, whereas others use longer recordings or smaller EEG segments simply categorized as either containing IEDs or not. If the number of IEDs per minute are not within the same range between datasets, measures such as the specificity, selectivity and the false detection rate cannot be compared in a fair manner. As suggested by Halford (2009), a multi-center project is needed to create a dataset for reliable benchmarks. The author later presents such a dataset, and although still under development, it may suffice for benchmark testing and future comparisons (Halford et al., 2011, 2012). Regardless of using different datasets, our method compares well to others. Depending on the confidence threshold chosen, the system achieves sensitivities of up to 0.99 . With thresholds chosen as to limit the false detection rate, a mean sen- 
sitivity of 0.90 and mean false detection rate of 2.36 is achieved. We feel that the threshold should be set interactively by the reviewer during runtime, which can start by viewing the most probable IEDs first with a high threshold value, and then gradually view more detections (with greater false detection rate) if needed by lowering it.

Apart from achieving high accuracies in epileptiform event detection, automated methods should also be responsible for presenting their findings to the reviewer in a fast and efficient manner. Although some commercial applications already exist, automated detection is mostly ignored by practicing neurologists. One reason for this could be that the software is still too complicated and time consuming to use. Fig. 7 shows an example of how a detected event is presented to a clinician. To make review time faster, events can be grouped by location or morphology and reviewing can take place on a macro scale to save time. This is already shown in some studies (and some products) who use clustering techniques to group similar looking events together (Nonclercq et al., 2012; Scherg et al., 2012; Ji et al., 2011a).

Although our method shows promising results, it is still far from ideal and some reported methods appear to perform better. Many of them however, have already been refined to the point where further improvement is limited. Our method, as presented here, is only a first step in testing a novel approach where many improvements can still be made. In work to follow, we will try to use more of the spatial context available to discard artifacts and lower the false detection rate. One way that we can achieve this is by introducing event clustering. Events with similar properties can be clustered, and each cluster's validity as IEDs can be determined based on the spatial distribution and frequency of occurrence of the events it contains. Apart from clustering, a number of ad hoc rules can also be applied as a post-detection step to mimic the procedures followed by reviewers when determining the validity of IEDs. Further work will also focus on testing the system on long-term EEG recordings such as in-home ambulatory monitoring, where the burden of visual inspection is even greater and more problematic for the reviewer.

In summary, automated detection of inter-ictal epileptiform activity will become invaluable to current reviewing techniques that rely mostly on visual analysis. A reliable system for a wide range of EEGs with different spike morphologies is needed, which should be tested with a gold-standard dataset for comparisons with other techniques. With minor improvements and an intuitive interface, the presented method is capable of providing more efficiency with reviewing and in turn improve the diagnostic efficiency of epilepsy. 



\section{Chapter 6}

\section{A Self-Adapting System for the Automated Detection of Inter-Ictal Epileptiform Discharges}

Shaun S. Lodder

Michel J. A. M. van Putten 


\begin{abstract}
Scalp EEG remains the standard clinical procedure for the diagnosis of epilepsy. Manual detection of inter-ictal epileptiform discharges (IEDs) is slow and cumbersome, and few automated methods are used to assist in practice. This is mostly due to low sensitivities, high false positive rates, or a lack of trust in the automated method. In this study we aim to find a solution that will make computer assisted detection more efficient than conventional methods, while preserving the detection certainty of a manual search. Our solution consists of two phases. First, a detection phase finds all events similar to epileptiform activity by using a large database of template waveforms. Individual template detections are combined to form "IED nominations", each with a corresponding certainty value based on the reliability of their contributing templates. The second phase uses the ten nominations with highest certainty and presents them to the reviewer one by one for confirmation. Confirmations are used to update certainty values of the remaining nominations, and another iteration is performed where ten nominations with the highest certainty are presented. This continues until the reviewer is satisfied with what has been seen. Reviewer feedback is also used to update template accuracies globally and improve future detections. Using the described method and fifteen evaluation EEGs (241 IEDs), one third of all inter-ictal events were shown after one iteration, half after two iterations, and 74\%, 90\%, and 95\% after 5, 10 and 15 iterations respectively. Reviewing fifteen iterations for the $20-30 \mathrm{~min}$ recordings took approximately 5 min.
\end{abstract}

Significance: The proposed method shows a practical approach for combining automated detection with visual searching for inter-ictal epileptiform activity. Further evaluation is needed to verify its clinical feasibility and measure the added value it presents. 


\subsection{Introduction}

Regardless of all the technological advances in recent years, routine scalp EEG is still used as the standard clinical procedure for diagnosing epilepsy. Not much has changed regarding visual analysis during diagnosis, even though computerized algorithms have been proposed to assist with reviewing (see (Wilson and Emerson, 2002; Halford, 2009) for review). The standard diagnostic strategy in a first-time seizure patient is to perform a routine 20-30 min scalp EEG recording and determine if any inter-ictal epileptiform discharges (IEDs) are present. IEDs appear in the raw signal in the form of spikes, sharp waves, poly-spikes, or spike and slow-wave discharges. Given that these patterns are correlated with a high likelihood of a recurrent seizure, their presence play an important role in the diagnosis of epilepsy.

Although the presence of IEDs are very indicative of epilepsy, their absence on the other hand does not exclude the disease. Patients are often required to return for a follow-up recording if the routine EEG is normal. Visual inspection combined with a manual search for IEDs is time consuming and requires an experienced electroencephalographer. Longer recordings and sleep-deprived EEGs have shown to improve the chances of finding inter-ictal activity and thereby yield higher diagnostic efficiency (Faulkner et al., 2012; Agbenu et al., 2012; Leach et al., 2006; Friedman and Hirsch, 2009), but given the visual burden already with shorter recordings, this is difficult to implement on a routine basis. Beyond the diagnosis of epilepsy, there is also a need to accurately mark epileptiform activity and investigate properties such as the potential seizure focus and epilepsy type.

Computer-assisted IED detection can help reviewers to find relevant information and reduce the burden of visual analysis. By finding all possible inter-ictal events and presenting them to the reviewer, a page-by-page inspection of the recording can be avoided. Two relevant measures of performance for automated spike detection methods are (i) their sensitivity to find IEDs, and (ii) the average false detection rate. If the sensitivity is too low, a reviewer cannot count on the results of the automated analysis alone to exclude IEDs from the recording. On the other hand, if the false detection rate of an algorithm is too high, the reviewer will spend more time filtering through detected events than by simply performing a visual review himself.

Many detection algorithms have been proposed and a wide range of techniques have been suggested. Recent studies have focused on either one or a combination of the following approaches: parametric methods; mimetic analysis; Fourier 
or wavelet analysis; artificial neural networks; template matching; context-based rules; and event clustering (Scherg et al., 2012; Nonclercq et al., 2012, 2009; Halford et al., 2012; Ji et al., 2011a; Wang et al., 2010; De Lucia et al., 2008; Indiradevi et al., 2008; Zhou et al., 2012; Lodder et al., 2013; Argoud et al., 2006; Subasi, 2006; Inan and Kuntalp, 2007; Van Hese et al., 2008; Kumar et al., 2012; Shen et al., 2013). Clearly, a lot of thought has been given to effective ways of detecting inter-ictal activity. However, almost all of these methods require thresholding to separate IEDs from other events in some way or another, making them limited in achieving both high sensitivities and low false detection rates at the same time. Low thresholds will result in high sensitivities with many false detections, whereas high thresholds will reduce the number of false detections but also lower the sensitivity.

Instead of simply searching for another spike detection technique, this study focused on finding a practical approach to combining automated detection with manual searching. This was achieved by bringing together the detection and review phases with the help of detection certainty values for each event, and updating detected event certainties with the help of user feedback. With our approach, we were able to circumvent the limitation imposed by detection algorithms where a threshold value has to be chosen to achieve either high sensitivities or low false positive rates. By design, the described system learns from the feedback it receives and updates the certainty values of the remaining nominations to prioritize more likely events. In addition, the feedback is also used to update the system on a global level, which aims to improve future detections and reviews.

The ultimate goal of this system is to improve reviewing accuracy and reduce standard review times to below the average of manual detection.

\subsection{Methods}

This section is divided into two parts. The first part reviews the template-based spike detection algorithm described in (Lodder et al., 2013) which we also use in this study, and summarizes the main steps needed to obtain a collection of detections which serve as "nominated" inter-ictal events. The second part explains how these nominations are grouped together and presented to the reviewer for verification, and how the proposed system can adapt during verification to learn from reviewer feedback. The template-based detection method was chosen for two reasons. First, by using a wide range of IED samples extracted from EEG training data and using the principle of voting and reliability to prioritize detections, the system is able 
to scale to any recording given that the IEDs it contains are similar in morphology than the templates in the database. Secondly, by using this technique, we were able to assign a certainty value to each detection which enabled us to sort the events in decreasing order of likelihood.

\subsubsection{Template-based IED detection and nominating inter-ictal events}

\section{Collection and training of a template database}

To locate possible IEDs in an EEG recording, a database of templates is used to find high correlations with events that represent inter-ictal epileptiform activity. Each template represents a sample IED waveform, and together with this the number of detections it has made in the past are stored including the outcomes of these detections, i.e. true or false positive. The database requires training only once with a training dataset and can thereafter be used on any new EEG to find inter-ictal epileptiform events. For building a database of IED template waveforms, a training set of eight EEGs were used with a total recording length of $175 \mathrm{~min}$. For the training set, an experienced electroencephalographer (MvP) was asked to mark all inter-ictal events in each recording where a clear spike-wave discharge could be seen. Spike-wave events were marked individually on all channels where they were visible. The review took place in three montages: common reference, bi-polar, and Laplace. The training dataset consisted out of 482 inter-ictal events, and from them a total of 2973 spike-wave samples were marked across all channels and montages. Using these marked events as our templates, a database was created where each underlying epoch of data was extracted and stored. Templates ranged from 212 to 860 milliseconds in length. Some sample waveforms are shown in Fig. 1.

After extracting the templates, their ability to find and discriminate between other IEDs from the same or other recordings and non-epileptiform activity was determined. This was done by finding a time-shifted correlation between each template and all EEG channels for all EEGs in the training set with the same montage. Locations were found where the templates had correlations above 0.85 , and the underlying EEG segment was extracted to calculate additional properties to further determine the relationship between itself and the template by which it was detected. These properties related to matching variance, amplitude differences, and the ratio between the detected epoch and EEG segments preceding it. For a detailed description of the properties, see (Lodder et al., 2013). Using the correlations between the detected epochs and the template, together with the additional prop- 

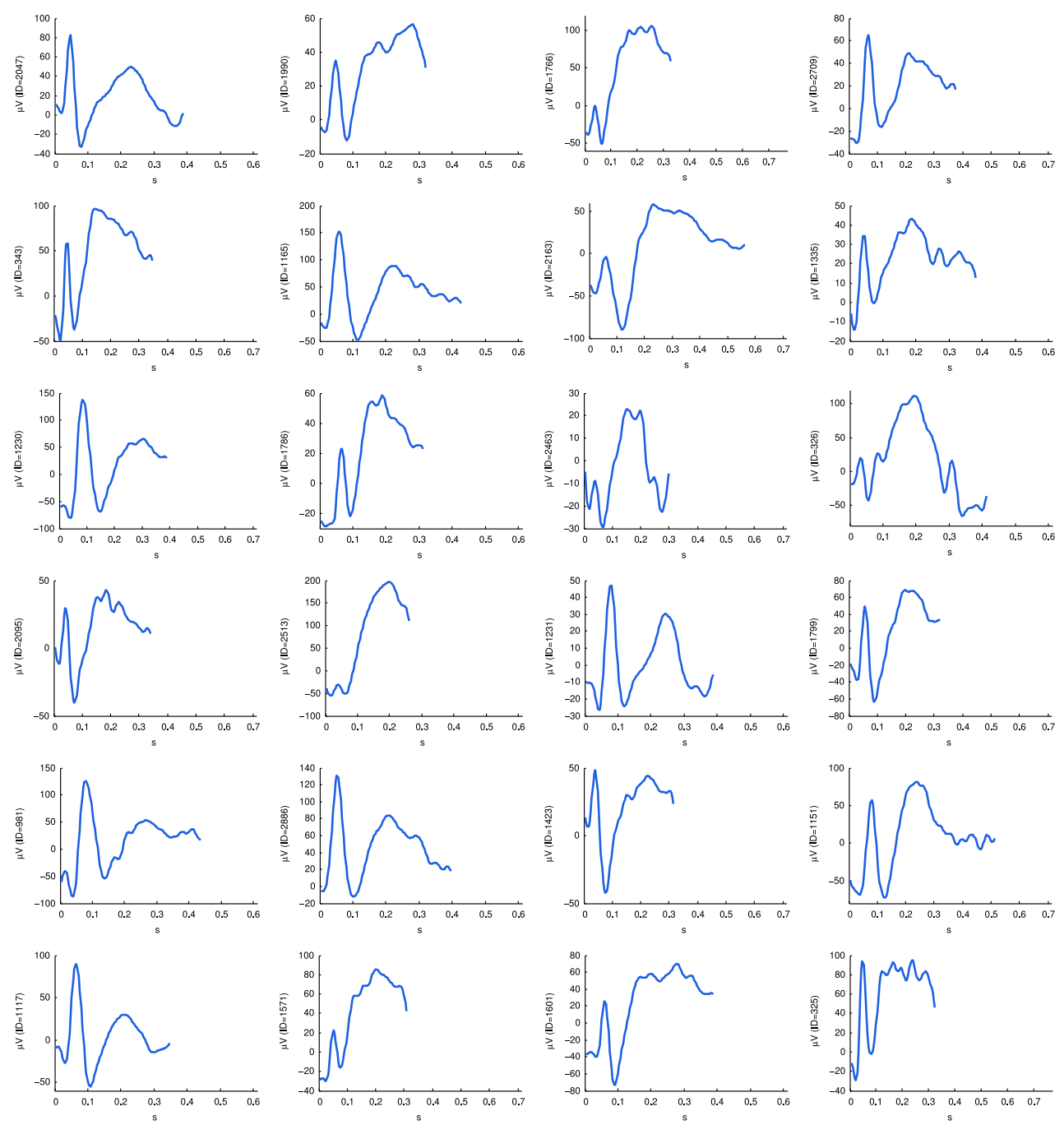

Fig. 1: Sample template waveforms collected from a training dataset. To find inter-ictal epileptiform activity in new unseen EEGs, each template searches for matching waveforms with high correlations to themselves. Thereafter, individual template detections are combined to form "IED nominations" which are then presented to the reviewer in decreasing order of likelihood.

erties and a known outcome for each detection, a linear support vector machine (SVM) was trained with these parameters as features to learn the difference between true and false detections. The trained SVM for each template together with its score of true and false positives was stored in the database for further use during detection. During the training phase, templates were discarded if they had zero 
detections, only false detections, or a small number of correct detections and no false detections. After discarding unwanted templates, a total of 2256 remained.

\section{IED detection in new EEG recordings}

For the detection of inter-ictal activity in new recordings, a similar procedure was used as during learning: for each template in the database, a time-shifted correlation is calculated on every EEG channel in three montages: common reference, bi-polar and Laplace. Epochs with correlations above 0.85 are "nominated" as IED events if they satisfy both the property constraints for that template and it's SVM classifies it as an IED. Each nomination is also assigned a reliability value, which is calculated as TPs/(TPs $+F P s)$, where TPs and FPs are the total number of true and false predictions that the template has made over all time (note that these scores are saved together with each template in the database).

\subsubsection{Using IED nominations to find inter-ictal events}

Our main goal in this study was to combine automated methods with visual reviews to obtain a clinically feasible method for computer-assisted detection of interictal epileptiform discharges. By not discarding any events or using any thresholds during the detection phase, the system was not optimized for achieving a high specificity. Instead, our focus was on providing the reviewer with as many interictal events as possible within a reasonable amount of time. By using an efficient algorithm to present the reviewer with the most probable events first, it is not required of him to review all the detected events in order to draw informed conclusions. Given that the review process is interactive and that no thresholds are used, many of the nominated events will be false positives which are not necessarily presented to the reviewer and ROC curves cannot be used fairly to evaluate the benefits of such a system. Instead, we aim to measure its performance by the percentage of IEDs in a recording that can be shown within a feasible amount of time or minimum number of iterations.

To graphically illustrate how the adaptive reviewing method works, a flow diagram of the described system is shown in Fig. 2. As a first step, all IED nominations are found using the template-based IED detection method described in the previous section. As shown in the diagram, the detection phase can be performed independently at any time before the EEG review takes place. Using Fourier optimizations to calculate the correlations (circular convolution), this takes approximately 12-14 
min for a 20-30 min recording. This can occur directly after the recording, after an upload to the hospital database, or even in real-time during acquisition. Because very few reviews will occur directly after a routine recording in practice and that this step can be completely automated, it can be considered as part of the acquisition and preprocessing step, and not as part of the actual review time for the electroencephalographer.

\section{Grouping}

When the computer-assisted review starts, all IED nominations from individual templates are loaded and overlapping events are merged to form grouped nominations. This is illustrated in Fig. 3. Merging takes place in two steps. First, overlap-

ping template nominations are combined on the same channel if they overlap by more than $75 \%$. Grouped events with fewer than three nominations are discarded. Next, overlapping groups over different channels are merged together where their onsets start within one second from the first item in the group. After these two steps, a number of grouped nominations have been obtained which point to segments in time where possible inter-ictal activity may exist.

\section{Group presentation combined with reviewer feedback}

If the entire list of group nominations were to be shown to a reviewer, more than $99 \%$ of the inter-ictal discharges in each recording will be found (see results (Lodder et al., 2013)). However, this method has the disadvantage of also having a very high false positive rate, and without a smart way of presenting the detections, the reviewer is better off searching for IEDs manually. Fortunately, and what also sets this method apart from other techniques, is that each grouped nomination has an assigned certainty value based on a combination of two factors: (i) the correlation of each contributing template to the detected segment, and (ii) the reliability of those templates in making accurate detections. The calculation of each nomination group's certainty value is shown in the Appendix. Using the certainty value to the system's advantage, a limited number of nominations with high likelihoods are shown to the reviewer, and in an iterative manner, more can be requested until the reviewer is satisfied with what has been seen. By presenting the nominated events in iterations, the system is able to receive feedback during the review. This allows it to update the reliability of the templates and thereby show more reliable detections first. 


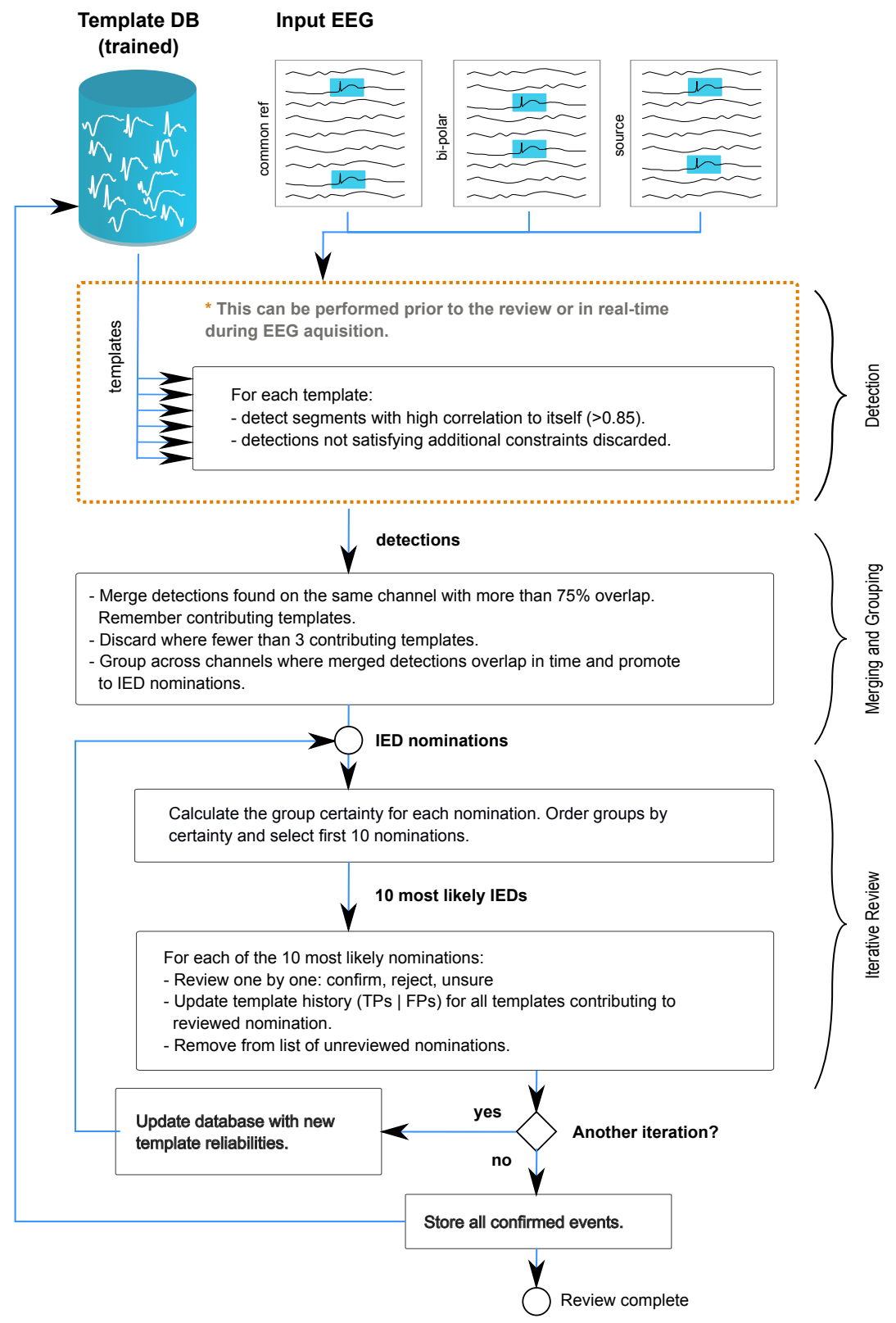

Fig. 2: Outline of the IED detection, grouping, and presentation steps. Multiple detections of interictal activity are made using a database of matching template waveforms. Individual template detections are merged and grouped (see Fig. 3) to form IED nominations, and the nominations are presented for review in an iterative manner, ordered by nomination certainty. 


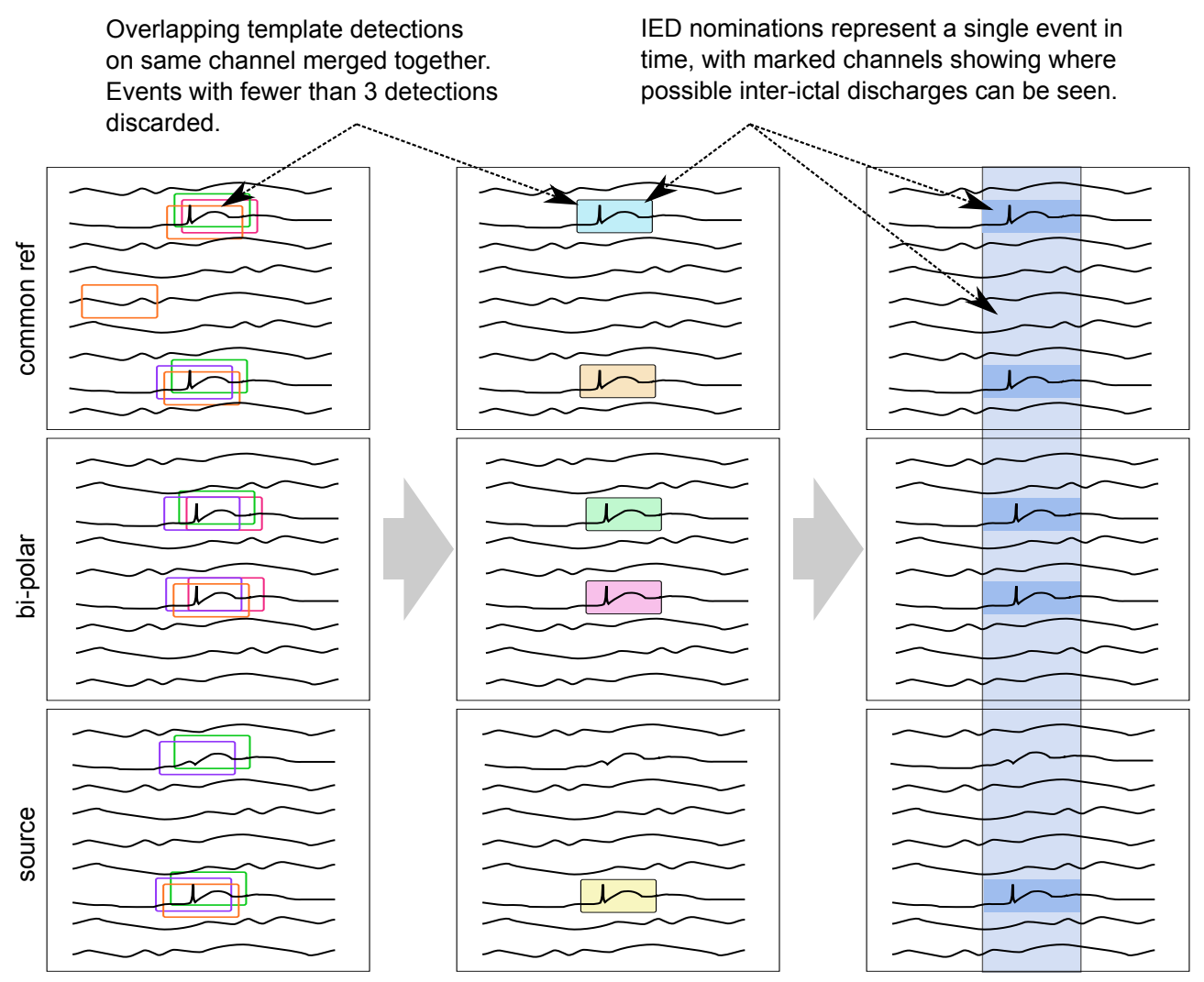

Fig. 3: Grouping and merging of template detections. Overlapping templates are merged together and grouped to form single IED nominations, which are presented to the reviewer as a single event in time. Channels containing template detections are highlighted to point out where inter-ictal activity was found.

Implementation of the described procedure is as follows: Using the entire pool of nominations obtained from the merging and grouping phase, group certainty values are calculated for each nomination. The ten nominations with highest group certainties are then shown to the reviewer one at a time, and the reviewer is given the chance to either confirm the nomination as an IED, reject it, or label it as unsure. After the ten nominations have been graded, their outcomes are returned to the system and the reliabilities of the contributing templates are updated. Given that template reliabilities affect the group certainties of each IED nomination (see Appendix), the group certainties of the remaining nominations are updated. Thereafter, another iteration takes place where ten of the remaining group nominations with highest certainties are shown. This process continues until the reviewer is 
satisfied with the events seen or until all nominations have been shown. When the review is complete, all confirmed nominations are stored and marked in the recording as detected IEDs.

\section{Global update for sustained learning}

Apart from making the review of automated detection more efficient by showing the most probable nominations first, this method also allows the system to implement a globally adaptive technique for improving future detections. After an EEG review is complete, the template database is updated to include the additional number of true and false positives made by each template. As described above and also shown in the Appendix, template reliabilities are measured by the number of true and false positives made in the past. Given that more classifications are added over time, a more accurate measure of each template's reliability can be derived. Therefore, over time with continuous use of this technique, reliable templates will become more reliable and have more influence during detection, whereas less reliable templates will receive a lower weight and have less influence.

\subsubsection{Subjects and data}

A dataset from the department of Clinical Neurophysiology at the Medisch Spectrum Twente (MST) Hospital in the Netherlands was used to evaluate the proposed method. All EEG data were obtained as part of routine patient care and anonymized before use. Our evaluation dataset consisted of 15 EEGs with a total recording length of 306 min and 241 marked IEDs. The inter-ictal events were marked prior to our evaluation by an experienced electroencephalographer (MvP), and given the time consuming nature of this, only the onset and duration of IEDs were marked and not specific channel information. Table 1 provides additional information about each recording in the evaluation set. Recordings were made using a standard 20-30 minute protocol with the Brainlab EEG system. $\mathrm{Ag}-\mathrm{AgCl}$ electrode caps were used with electrodes placed according to the 10-20 system, and impedances were kept below $5 \mathrm{k} \Omega$ to reduce polarization effects. Recordings were made at a sample rate of either $250 \mathrm{~Hz}$ or $256 \mathrm{~Hz}$ and band-pass filtered between $0.5-30 \mathrm{~Hz}$. The data was downsampled to $100 \mathrm{~Hz}$ to increase the performance of the system, and eye blink artifacts were reduced using an independent component analysis filter. 


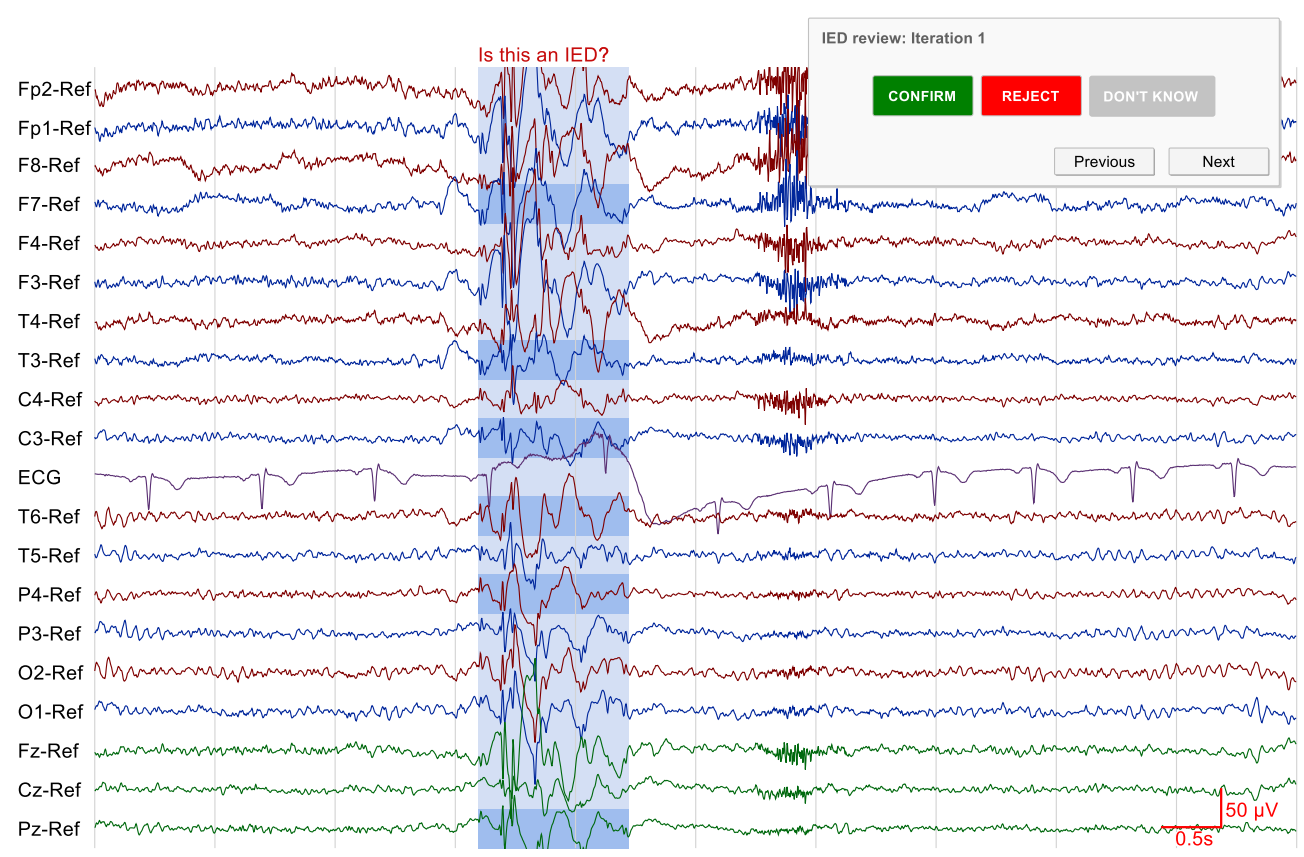

Fig. 4: A screenshot showing how the review process works. For each iteration, the reviewer is presented with 10 nominations, one at a time, and is given the opportunity to either confirm, reject, or ignore each event as inter-ictal epileptiform activity (see top right panel). After the ten nominations have been graded, the user can choose to either stop with the review or to review another ten events. Nomination certainties are updated between iterations.

\subsection{Results}

Using the described method, IED nominations were found for all of the 241 IEDs in the evaluation dataset. Given however that no thresholds were used to discard events with low certainties, the system would have a very high false positive rate if all nominations had to be shown to a reviewer. As described in the Methods, iterative reviewing is introduced to help minimize the number of presented false positives. An example of the review process is shown in Fig. 4. A software application is used to show 10 seconds of EEG at a time with the nominated event centered in the middle of the screen. The grouped nomination is highlighted to indicate its onset and duration, and additional highlights are placed on the channels where the templates have found possible IED waveforms. The reviewer is presented with three options in the top-right corner of the screen. From these options the reviewer can decide to either confirm, reject, of ignore the displayed nomination as an IED. The simplicity of this approach allows the user to evaluate nominations in a fast 


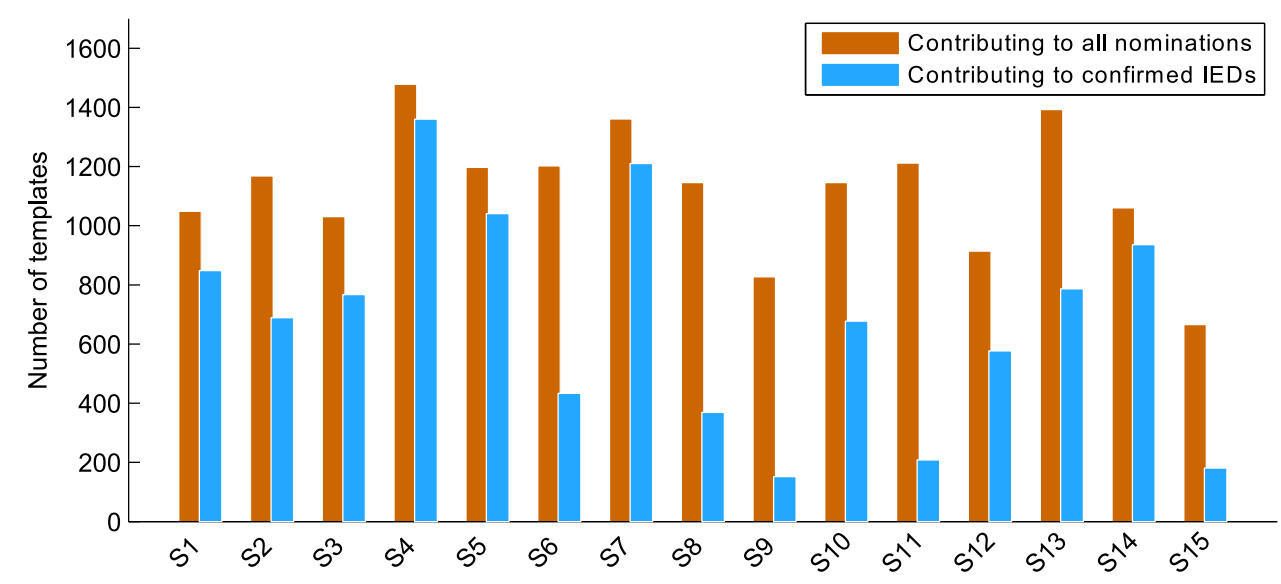

Fig. 5: Template contributions per recording: The first bar shows the number of templates that have contributed to all the nominations presented during a review of fifteen iterations. The second bar shows the number of templates that contributed to confirmed IEDs only.

and effective manner. Although only measured during the development of this algorithm, the average duration of visually reviewing one iteration consisting of ten nominations was approximately 20 seconds.

Table 2 shows the number of IEDs detected per recording for each iteration during the review. To provide consistency during the evaluation, nominations were confirmed by comparing them to the marked IEDs obtained earlier from visual inspection. The first and second columns provide the subject ids and number of marked IEDs, and the remaining columns show the number of confirmed IEDs after each iteration of the review. Fifteen iterations were used to evaluate the method with ten nominations presented per iteration. We see that approximately one third of all IEDs are shown within the first iteration of the review and already half of them after two iterations. After five iterations $74 \%$ of the events were found, and with ten and fifteen iterations the number of detected IEDs increased to $90 \%$ and 95\% respectively.

Figure 5 provides some insight into the number of templates involved during detection. A bar graph is shown consisting of two bars per subject. The first bar shows the number of templates contributing to all nominations presented during the review of fifteen iterations. The second bar shows the number of templates that contributed to confirmed IEDs. From this we see that a substantial number of templates are involved during detection, and more importantly, that many of them 
Table 1: Subject information of the EEGs used to test the detection and reviewing method.

\begin{tabular}{llll}
\hline Subject & Epilepsy type & Age (years) & Duration (min) \\
S1 & generalized idiopathic & 45 & $16: 19$ \\
S2 & absence & 26 & $20: 00$ \\
S3 & absence & 10 & $21: 30$ \\
S4 & generalized other & 50 & $20: 19$ \\
S5 & generalized other & 7 & $20: 40$ \\
S6 & temporal lobe & 51 & $21: 00$ \\
S7 & generalized other & 44 & $20: 00$ \\
S8 & generalized idiopathic & 12 & $22: 30$ \\
S9 & temporal lobe & 17 & $20: 00$ \\
S10 & generalized other & 10 & $20: 00$ \\
S11 & absence & 42 & $22: 19$ \\
S12 & generalized idiopathic & 10 & $19: 30$ \\
S13 & temporal lobe & 7 & $20: 19$ \\
S14 & generalized idiopathic & 4 & $21: 10$ \\
S15 & generalized other & 44 & $20: 00$ \\
\hline
\end{tabular}

contribute to confirmed IEDs. We also see that many templates are responsible for negative contributions. It should be noted however that fifteen iterations were used per review, and that in most recordings, only a small number of inter-ictal events were present. This means that if all IEDs were confirmed, the system would only have false positives left to show. This figure also confirms that a sufficient number of template waveforms were available in the database to detect IEDs in the new, unseen EEGs of our evaluation dataset. Template reliabilities are not reflected in this graph, meaning that detections with high certainty are not separated from those with low certainty. Given the average reviewing time to perform one iteration, a typical review of fifteen iterations will approximately last five minutes for a 2030min EEG recording. This estimate is however only based on internal evaluation, and a larger study with multiple participants is required to obtain an accurate measure. The review time will be reduced even further if a reviewer is already satisfied with the nominations seen after fewer iterations.

\subsection{Discussion}

A large number of inter-ictal spike detection methods have been proposed in recent years (see (Halford, 2009; Wilson and Emerson, 2002) for review). Many of these have been designed with patient specific features, or have been tested on segmented EEG data with possibly reduced movement and eyeblink artifacts. For these 
reasons alone, they already appear not to scale well to new, unseen EEG recordings as we would wish for practical consideration. Given the nature of spike detection algorithms, it is also difficult to achieve both a high sensitivity and a low false detection rate simultaneously. Some studies therefore place their focus on achieving high sensitivities (Nonclercq et al., 2012; Argoud et al., 2006; Subasi, 2006; Halford et al., 2012; Van Hese et al., 2008), whereas others aim to maintain low false positive rates (De Lucia et al., 2008; Ji et al., 2011a; Indiradevi et al., 2008). In a practical scenario, this means that reviewers will be forced to look at a large number false positive events, or to see fewer nominations and miss out on important detections. When only these two options are available, it is clear to see why epileptologists prefer the more conventional method of visual inspection with manual detection. Our system has been optimized to achieve high sensitivities with events being presented to the reviewer in a minimal amount of time. A weakness in the system therefore lies in a higher number of false positives which range from 0.24-6.6 fp/min depending on the number of iterations chosen. Sensitivities for other reported methods range from 0.5 to 0.92 and false detection rates from 0.1 to $6 \mathrm{fp} / \mathrm{min}$ (Halford, 2009; Wilson and Emerson, 2002).

This study presents an alternative approach to inter-ictal spike detection and review. It has three fundamental differences to other automated methods, which to our knowledge has not been used before: First, individual IED nominations are assigned a detection certainty, giving the system an opportunity to present them in a descending order of likelihood. Secondly, the described system can learn from the outcome of events that have been presented, and in such a way attempt to increase its sensitivity during presentation of the remaining nominations after every iteration. Lastly, the system can adapt itself on a continuous and universal basis, thereby improving its own accuracy over time. By storing the number of true and false positive detections of each template and updating their reliabilities accordingly, the template reliabilities will become more accurate which in turn will improve the overall robustness of the system.

Given that IED nominations are presented in a descending order of likelihood, the reviewer is not required to view all nominations before knowing if any interictal activity exists. Instead, it is left up to the reviewer to decide on the number of nominations to view before a diagnosis is made. Because of this, the system is less vulnerable to high false positive rates. This approach also avoids the need for thresholding to limit the number of nominations presented to the reviewer, and should make it scalable to long-term recordings. Further evaluation is required to 
Table 2: Adaptive iterative reviewing: IED nominations are iteratively presented to the reviewer one-by-one using 10 events per iteration. Nomination certainties are updated after every iteration, thereby allowing nominations with higher likelihoods to be shown first. Template reliabilities were also updated at the end of each review. This was done by updating the true and false positives of contributing templates and recalculating their reliability scores accordingly, as shown in the Appendix.

\begin{tabular}{|c|c|c|c|c|c|c|c|c|c|c|c|c|c|c|c|c|}
\hline \multirow[t]{2}{*}{ Subject } & \multicolumn{5}{|c|}{ \#IEDs } & \multicolumn{11}{|c|}{ IEDs confirmed up and until iteration } \\
\hline & & 1 & 2 & 3 & 4 & 5 & 6 & 7 & 8 & 9 & 10 & 11 & 12 & 13 & 14 & 15 \\
\hline S1 & 13 & 8 & 11 & 13 & 13 & 13 & 13 & 13 & 13 & 13 & 13 & 13 & 13 & 13 & 13 & 13 \\
\hline S2 & 11 & 4 & 6 & 6 & 8 & 8 & 8 & 8 & 8 & 9 & 9 & 9 & 9 & 9 & 9 & 9 \\
\hline S3 & 5 & 5 & 5 & 5 & 5 & 5 & 5 & 5 & 5 & 5 & 5 & 5 & 5 & 5 & 5 & 5 \\
\hline S4 & 36 & 4 & 8 & 14 & 17 & 20 & 24 & 27 & 29 & 30 & 31 & 32 & 32 & 33 & 33 & 35 \\
\hline S5 & 19 & 8 & 12 & 15 & 18 & 19 & 19 & 19 & 19 & 19 & 19 & 19 & 19 & 19 & 19 & 19 \\
\hline S6 & 6 & 4 & 5 & 5 & 5 & 5 & 5 & 5 & 5 & 5 & 5 & 5 & 5 & 6 & 6 & 6 \\
\hline S7 & 79 & 9 & 17 & 26 & 36 & 45 & 52 & 58 & 61 & 62 & 68 & 68 & 70 & 72 & 74 & 74 \\
\hline S8 & 5 & 3 & 4 & 4 & 4 & 4 & 4 & 4 & 4 & 4 & 4 & 4 & 5 & 5 & 5 & 5 \\
\hline S9 & 2 & 2 & 2 & 2 & 2 & 2 & 2 & 2 & 2 & 2 & 2 & 2 & 2 & 2 & 2 & 2 \\
\hline $\mathrm{S} 10$ & 12 & 3 & 10 & 11 & 11 & 12 & 12 & 12 & 12 & 12 & 12 & 12 & 12 & 12 & 12 & 12 \\
\hline S11 & 7 & 1 & 2 & 2 & 2 & 2 & 2 & 2 & 2 & 2 & 2 & 2 & 2 & 2 & 2 & 2 \\
\hline $\mathrm{S} 12$ & 7 & 3 & 4 & 5 & 6 & 6 & 6 & 7 & 7 & 7 & 7 & 7 & 7 & 7 & 7 & 7 \\
\hline S13 & 19 & 10 & 17 & 19 & 19 & 19 & 19 & 19 & 19 & 19 & 19 & 19 & 19 & 19 & 19 & 19 \\
\hline S14 & 14 & 9 & 12 & 12 & 12 & 13 & 14 & 14 & 14 & 14 & 14 & 14 & 14 & 14 & 14 & 14 \\
\hline S15 & 6 & 5 & 5 & 5 & 6 & 6 & 6 & 6 & 6 & 6 & 6 & 6 & 6 & 6 & 6 & 6 \\
\hline TOTAL & 241 & 78 & 120 & 144 & 164 & 179 & 191 & 201 & 206 & 209 & 216 & 217 & 220 & 224 & 226 & 228 \\
\hline MEAN & & $32 \%$ & $50 \%$ & $60 \%$ & $68 \%$ & $74 \%$ & $79 \%$ & $83 \%$ & $85 \%$ & $87 \%$ & $90 \%$ & $90 \%$ & $91 \%$ & $93 \%$ & $94 \%$ & $95 \%$ \\
\hline
\end{tabular}

verify this.

Some methods try to overcome the burden on visual confirmation by clustering nominations together into groups based on similarity in space and morphology (Van Hese et al., 2008; Nonclercq et al., 2012; Scherg et al., 2012). Few studies have given detailed descriptions on how detected IEDs are presented to reviewers, but the most similar technique to ours is given by (Scherg et al., 2012). In their method, the authors describe a system that uses clustering to group events based on a similarity measure calculated from a subset of 18 quantitative features. Their system orders the clusters by frequency of occurrence and presents the reviewer with one cluster at a time to review. The reviewer can confirm which clusters contain IEDs, and by confirming a cluster, all events contained within it are labeled as IEDs. Although this technique is similar to ours in some ways, a number of key differences exist. First, given that IEDs are confirmed in clusters and not as single 
events, the cluster-based review approach is vulnerable to confirming false detections clustered together with true IEDs. Although in our method the confirmation of single events will potentially take longer than confirming clustered events, the reviewer is assured that all marked IEDs will have been shown and are in fact true inter-ictal events. The second key difference is that, apart from ordering the clusters by frequency of occurrence, the cluster-based system does not have any certainty measure similar to the one we describe. All clusters therefore need reviewing in order not to miss important events, and this can result in longer review times. Lastly, our system is unique in incorporating reviewer feedback from current reviews to improve the detections on future recordings.

As with any method, a number of improvements can still be made to further increase the efficiency and accuracy of our technique. Specific areas of improvement include a built-in mechanism to collect and train additional templates for expanding the template database with more example waveforms. This will result in a completely closed-loop system, where better templates can be promoted over time and unreliable templates discarded. Additionally, context-based rules such as those described by (Scherg et al., 2012; Argoud et al., 2006; Ji et al., 2011a; Van Hese et al., 2008; Nonclercq et al., 2012) can be added to group nominations according to their similarity, which can be used to provide additional information during the review. Given that only 15 EEGs were used to evaluate this method and that reviewer bias may have been added by having the IEDs marked by only one reviewer, further evaluation is needed to determine the feasibility of this method in clinical practice. Further work will involve multiple electroencephalographers and a comparison between visual searching and automated detection in long-term recordings.

In summary, by adding information to detected events in the form of certainty values, nominated IEDs can be shown in a decreasing order of likelihood and thereby make it possible for the reviewer to view fewer events. In practical terms, this has the potential to improve efficiency and lower EEG review times, and thereby make automated detection faster than conventional manual reviews while achieving a similar diagnostic certainty.

\section{Appendix: Calculation of the group certainties}

Using the same method as described in (Lodder et al., 2013), a database of stored template waveforms make individual detections of inter-ictal activity by finding events in the EEG that have high correlations to themselves. Individual detections 
are merged and grouped where they overlap, and from this the grouped IED nominations are formed (see illustration Fig. 3).

Let each individual template detection be defined as

$$
D_{\text {tem }}=\left\{C R R_{\text {tem }} ; T P_{\text {tem }} ; F P_{\text {tem }} ; R_{\text {tem }}\right\},
$$

where $C R R_{\text {tem }}$ is the correlation coefficient between the template and the detected segment, $T P_{\text {tem }}$ and $F P_{\text {tem }}$ the total number of true and false detections made by that template over all time (stored in the database), and $R_{t e m}$ a measure of the template reliability:

$$
R_{\text {tem }}=\frac{T P_{t e m}}{T P_{t e m}+F P_{t e m}} .
$$

Given that each IED nomination can be seen as a combination of $N$ overlapping detections $\left(\right.$ nom $=\left\{D_{\text {tem }}\right\}$, tem $\left.\in[1 \ldots N]\right)$, the certainty value of that nomination is calculated as

$$
C E R T A I N T Y_{\text {nom }}=\max \left(\left\{C R R_{\text {tem }} \times R_{\text {tem }}\right\}\right), \text { tem } \in \text { nom } .
$$


Chapter 7

Summary

General discussion and

Outlook 

An estimated forty to sixty thousand routine EEG recordings are made in the Netherlands each year alone, and due to its portability and lower costs relative to other non-invasive procedures for functional neuroimaging, this number is growing steadily. With more recordings however, we are also presented with more data to analyze, and because of the time-consuming nature, training, and costs involved in performing these reviews, conventional methods of analyzing and reviewing EEGs are becoming problematic.

This thesis has explored the use of computerized interpretation to assist reviewers in analyzing EEGs faster and more consistently with the aid of quantitative and automated methods. Although EEGs are used in many areas of diagnostics and monitoring, this thesis has focused on routine clinical outpatient recordings and on the standard reviews performed for the diagnosis of epilepsy. Two areas of automated analysis were considered: the analysis of background activity and inter-ictal epileptiform spike detection. A summary of our contributions to each of these areas is listed below, followed by a discussion and outlook into further developments of automated EEG interpretation and the role it will play in the future of diagnostics and neuromonitoring.

\section{Summary}

\section{Part I - EEG background pattern}

For analyzing the various properties of the EEG background pattern, this study started with finding an accurate technique to automatically estimate the posterior dominant rhythm (PDR). Although easily measured in most healthy patients, the simple approach of calculating a mean spectrum over the eyes-closed EEG often failed due to artifacts or a weak presence of the PDR. More accurate and robust estimates were found by splitting the eye-closed EEG into smaller segments and then estimating peak frequencies in each of these segments individually. Chapter 2 describes a method in which this approach was implemented with the use of a curvefitting algorithm and clustering to combine the segmented estimates. To evaluate the technique, PDR frequency estimates were calculated from 1215 test EEGs. Diagnostic reports were available for all recordings, allowing us to extract and compare reported visual estimates to those obtained from the algorithm. Agreements with visual inspection ranged from $96 \%$ to $99.4 \%$ for a fault tolerance of $1.2 \mathrm{~Hz}$.

After finding an accurate technique to robustly measure the PDR frequency in an unsupervised manner, the next aim was to further describe other properties of 
the EEG background pattern. An additional four properties were chosen: the PDR reactivity, anterior-posterior gradients, left-right symmetry, and the presence or absence of abnormal diffuse slow-wave activity. In order to compare quantitative interpretation to visual reviews, 384 EEGs were gathered from a database at the MST hospital in the Netherlands in which all these properties were reported. During this process, it was found that the descriptions were often vague or inconsistent due to its free-text nature, and that no formal guidelines existed for describing some of the background properties. It was therefore decided that in addition to developing quantitative measures for describing these properties, we would create simple yet useful guidelines for reviewers to follow while noting down their findings in the diagnostic reports. These guidelines also served as definitions on which the quantitative algorithms could base their analysis, making the computerized interpretation transparent to the reviewer. Chapter 3 provides the reported guidelines, together with quantitative measures for each of the background properties considered. To test the reliability of these measures, the properties obtained from automated analysis were compared to the properties reported through visual inspection in the diagnostic reports. Some such as the PDR frequency, reactivity, and presence of diffuse slow waves were in moderate to high agreement with visual reports. For symmetry and anterior-posterior gradients, the agreement was lower. It was noted though that based on the vague free-text descriptions, these properties were difficult to place in set categories for comparison.

Given that the automated interpretation methods showed promising results, it was possible to further test them in clinical practice. For this, they were added into a custom-built EEG viewer, and together with a new evaluation set of 45 EEG recordings taken from the MST hospital database in the Netherlands, it was given to nine EEG experts across the Netherlands for evaluation, as described in Chapter 4. The EEGs varied enough to contain examples of both normal and abnormal occurrences of each background property considered. Participants were asked to first visually score each EEG, and then compare their findings with the interpretation provided by the system. Afterwards, the inter-rater agreement was measured for both the automated system and each participant using a gold-standard derived from the consensus of all reviewer reports. Automated interpretation together with most participants showed high (kappa $>0.6$ ) agreement with the gold standard, and in some cases, automated analysis showed even higher agreement with the gold standard than some participants. To determine the acceptance of such algorithms during routine reviews, the participants were also asked whether they would 
find it useful to have quantitative analysis methods assist them on a routine basis. For this, all participants answered yes.

From the studies related to automated analysis of the background pattern, we conclude that computer-assisted interpretation can have a significant contribution to routine EEG reviews. Although only tested on EEGs 20-30 min in length, the algorithms may also be useful during the screening of longer EEGs, including ambulatory recordings and ICU monitoring. Further work may help to improve interpretation accuracy and include more properties for review.

\section{Part II - Inter-ictal epileptiform discharges}

For routine outpatient visits, one of the main clinical reasons for EEG recordings is the diagnosis of epilepsy. A large part of this thesis was on finding a method that could automatically detect inter-ictal epileptiform discharges and present them to the reviewer without him having to visually screen the entire EEG first. A large number of spike detection algorithms already exist in literature without clinical implementations, and our focus was therefore not only on finding a suitable technique, but also to create a method which could become a practical solution for clinical use.

As a contribution to the automated detection of epileptiform activity, two studies were performed. The first, presented in Chapter 5, describes a technique that finds epileptiform discharges by comparing all signal waveforms present in the EEG to a trained database of sample discharges. In this chapter, we describe how 2160 templates are extracted from a group of training EEGs, and trained in classifying similar waveforms by allowing them to nominate epileptiform activity in an EEG based on the similarity to themselves. Templates are given a measure of reliability, and in combination with their own certainty of a detection, a voting system is used to group and merge individual template nominations together. Although formal comparisons could not be made due to different datasets, it was shown in Chapter 5 that the proposed method can achieve similar sensitivities and false detection rates as other techniques reported in literature. Due to not discarding many template nominations, it has more false detections at high sensitivities however, therefore still leaving it impractical in clinical use as compared to a standard visual review. This chapter does however also show that the detection technique can generalize to new, unseen recordings, and it can therefore have clinical relevance if the number of false detections is reduced. 
The second step in detecting inter-ictal epileptiform activity was to achieve a practical and scalable solution for clinical use. The problem was approached from a different perspective, and instead of looking for thresholds to discard false detections, the described system assigns a detection certainty value to each detected event, and orders them all in decreasing order of certainty. Next, the system presents ten events to the reviewer at a time in an iterative manner, and asks for either a confirmation or rejection of each event that is shown. Using this as feedback, the system then learns which events are of higher importance and adjusts the certainty values of the remaining detections accordingly. By allowing the reviewer to choose when he is satisfied with what has been shown and when to stop the iterative process, the system provides a practical solution to routine reviews and can easily scale up to long-term recordings. In Chapter 6, the system was tested on 45 routine EEGs of approximately 20-30 min in length, and we show that after a single iteration of screening ten events, an average of already $32 \%$ of the inter-ictal discharges in the recordings were found. After five iterations, this increased to $74 \%$ and after fifteen iterations, which took an average reviewing time of five minutes, $95 \%$ of the inter-ictal events present were detected and presented to the reviewer.

To truly test the benefit and advantages that this technique can provide in clinical practice, another evaluation by expert electroencephalographers is needed. From the results obtained up to this point however, the proposed method and review strategy appears to have a positive outcome.

\section{General discussion and Outlook}

If automated interpretation will ever replace the human reviewer remains to be seen. One thing that is for certain is that the presence of quantitative EEG analysis will continue to grow during reviews. Many types of quantitative EEG features have been proposed to describe specific properties in the EEG, including statistical measures such as variance and kurtosis (Scherg et al., 2012; Stevenson et al., 2013), non-linear energy operators (Mukhopadhyay and Ray, 1998), smallworld networks and functional connectivity (Stam et al., 2007; Bullmore and Sporns, 2009), synchrony (Lachaux et al., 1999; van Putten, 2003), entropy (Stam, 2005; Kannathal et al., 2005), power ratios (Kurtz et al., 2009; Cloostermans et al., 2011), bispectral index (Sigl and Chamoun, 1994), and left-right symmetry (van Putten et al., 2004). Apart from epilepsy, clinical applications for quantitative EEG also include ICU monitoring (Friedman and Hirsch, 2010; Cloostermans et al., 2011; Foreman 
and Claassen, 2012), clinical psychiatry (Coburn et al., 2006; Hammond, 2010) and the diagnosis of neurodegenerative diseases (Petit et al., 2004; Babiloni et al., 2011; Moretti et al., 2012). Many differences exist between these fields, but quantitative analysis in all of them share a very similar goal: to reveal additional information about the patients neurological state and to make reviews more efficient and intuitive for clinicians. As shown throughout this thesis, our suggested use of automated methods is not to replace the reviewer, but instead to give him the additional tools needed to perform faster and more consistent reviews.

The algorithms presented here demonstrate how computational analysis brings additional value to visual interpretation. In Chapter 4 we have shown how computerassisted interpretation can help to improve inter-rater agreement and serve as a tool to present the background properties of an EEG to the reviewer in a more structured and intuitive manner. It was also shown how easy it can be to integrate computerassisted reviewing with standard visual analysis, and at the same time receive general acceptance from clinicians and their willingness to incorporate such a system into routine reviews. Although the study in Chapter 4 used automatically generated reports and asked participants to only make changes where they disagreed, other approaches can also be used to make assisted reviewing less intrusive for the reviewer. A different approach for example, will be to let clinicians fill out the report themselves and only ask the system to verify and point out properties for which it has a different outcome. By doing this, an additional verification step is added to reports which will surely help clinicians to improve their consistency and increase the overall inter-rater reliability.

Unfortunately, automated background analysis still has a number of weaknesses and the interpretations are limited to the number of features that the methods can describe. Regarding the background properties presented in Chapter 3 and 4, additional features of interest may include the interpretation of beta and gamma activity, mu rhythms, and the EEG response to photic stimulation and hyperventilation. Clear and intuitive ways of showing EEG properties using quantitative features are also required to encourage reviewers to use the provided tools. It is also important that computer-assisted systems are developed through continuous feedback loops between developers and clinicians to ensure that clinical applications remain relevant to the needs of clinicians.

Regarding inter-ictal epileptiform spike detection, we have taken a different route than many by rejecting black-box methods and instead developed a technique which more closely resembles humans and visual analysis. By having a technique that 
makes use of a template database to find matching inter-ictal discharges, actual samples of epileptiform activity have been used to train a computer on how to find inter-ictal discharges in the three most-commonly used montages, much in the same way as with training a clinician. Also, as with humans, the system is adaptive and can learn through its own mistakes, thereby improving upon itself over time.

The concepts and outline of our presented methods are very simple and transparent, although its implementation and the computational efficiency required to realize them needed a certain degree of complexity. This has lead to many areas where more improvements can be made. Two such areas for example, are in defining the adaptive behavior of the system, i.e. deciding how it learns over time, and the other is in matching the additional steps followed by clinicians when evaluating EEGs, e.g. taking into account medication and noting the frequency and spatial information of observed abnormalities. Also, to further improve epileptiform spike detection algorithms and compare them on a global basis, more collaboration is needed. Similar to the benchmark database of electrocardiographic recordings (Willems et al., 1985), a global benchmark for testing automated detection algorithms can be established by storing annotated EEGs in a standardized evaluation database. Halford et al. (2012) has presented such a database for EEG epileptiform spikes, and if it receives global acceptance, all algorithms can be evaluated in the same manner in the future to ensure fair and objective comparisons.

A major obstacle for quantitative EEG interpretation is the presence of artifacts. Some, such as movement or muscle artifacts, can completely corrupt the signal, whereas others may mimic pathological EEG, for example chewing or tooth brushing that may resemble epileptiform discharges. A large number of artifact detection and correction algorithms are available, but for increased accuracy in automated analysis, more development is needed to further reduce noise and improve the overall quality of these sensitive signals.

\section{Standardized reporting}

The EEG properties described in this thesis, in particular those in Chapter 3 and 4 , were chosen based on provided information available from diagnostic reports stored in a database at the MST hospital in the Netherlands. After reading through more than 2500 reports and annotating all properties reported in free-text, five background properties were found to be in most reports, and they were therefore chosen as a starting point for our study. Reading through this large amount of 
reports already proved by itself to be a tedious and time consuming task, but more importantly, it brought to light a major concern which others have also described. Free-text reports lack consistency, are difficult to query, often leave out important information, contain localized terminology not always understood by other groups, and sometimes have vague descriptions or ill-defined findings.

Fortunately, some EEG experts have taken on the task of standardizing EEG reports (Aurlien et al., 1999, 2004, 2007; Beniczky et al., 2013). The Standardized Computer-based Organized Reporting of EEG (SCORE), is a system designed to bring consistency and completeness to how EEG findings are annotated and stored. Based on the outcome of consensus workshops held in Europe from participants across 15 countries, standard terms and definitions for EEG findings have been agreed upon and organized categorically to be used for reporting (Beniczky et al., 2013). By providing set options for describing each EEG characteristic, the SCORE system tries to circumvent traditional free-text reports as much as possible, and thereby ensures consistency and universality to all EEG findings. This also allows institutions to more easily store, query and share their reports.

With minor modifications, the automated interpretation system presented in Chapter 3 and 4 can be modified to adhere to these terms and guidelines. In addition to the background properties, detected inter-ictal spikes which are presented to the reviewer as shown in Chapter 6, can also further be categorized and described according to the standard terms and definitions. For future development and the addition of more reported EEG properties by automated interpretation, SCORE provides a good reference as to which characteristics are most relevant.

\section{Towards computerized EEG interpretation in the future}

As shown in this thesis, there is still great room for improvement in automated EEG analysis and interpretation. Although the studies presented here have focused mainly on routine 20-30 min recordings, the use of EEG extends much further for both inpatient and outpatient settings. Improvements in hardware such as active electrodes, shielded wires and smaller amplifiers allow the technology to be used in environments which were not suitable before, and software advances including data synchronization and cloud computing create new possibilities for real-time monitoring and diagnosis. 


\section{Routine recordings and ambulatory $E E G$}

Given that EEG has become more portable and affordable over the past decade, new opportunities have emerged in the way that we monitor and diagnose patients (Askamp and van Putten, 2013; Waterhouse, 2003; Dash et al., 2012; Seneviratne et al., 2013). For epilepsy diagnostics, the relatively short recording length of 20-30 min routine recordings only provide a small window of opportunity for clinicians to capture inter-ictal events, leading to missed events and follow-up recordings. As shown by Faulkner et al. (2012) and Friedman and Hirsch (2009), longer recording windows can help to improve the chances of finding inter-ictal events if any exist. By using ambulatory monitoring and outpatient recordings, fewer hospital visits are required which can reduce healthcare costs significantly (Foley et al., 2000; Dash et al., 2012; Burke et al., 2013; Strzelczyk et al., 2012). For longer recording windows, computerized interpretation will play an important part in reducing the amount of data screened visually. Key properties and important events can be summarized, and presented to the reviewer in a shortened version of the recording or in a more intuitive manner, as demonstrated by in (Nakamura et al., 1992; Casson and Rodriguez-Villegas, 2009; Scherg et al., 2012; van Putten, 2008; Lodder and van Putten, 2013; Cloostermans et al., 2011; Kurtz et al., 2009; Moeller et al., 2011). Artifacts remain an important challenge in ambulatory EEG, where electrode-, muscleand movement artifacts can either corrupt the signal or imitate epileptiform behavior (Seneviratne et al., 2013).

Although ambulatory EEG can be great for many things, shorter recordings will still play an important role in clinical diagnosis. As shown in Chapter 3, 4 and 5, intuitive computerized methods can help to improve and simplify these reviews and thereby lead to higher efficiency and cost savings. Additionally, the added benefit of QEEG to calculate and display certain properties in an automated and simple manner will also allow less qualified staff such as nurses to obtain a general overview and interpretation of the patient's current neurological state.

\section{Neuromonitoring in the ICU}

Apart from epilepsy and sleep studies, EEG also has important uses in the intensive care unit (ICU). Patients suffering from subarachnoid hemorrhage, postanoxic coma after cardiac arrest, traumatic brain injury or any other serious type of encephalopathy will often benefit from continuous neuromonitoring during their stay in the ICU. Given that most of these patients are in a comatose state, an experi- 
enced neurointensivist is needed to monitor them on a continuous basis for nonconvulsive seizures (NCS), status epilepticus (NCSE), ischemia, or other abrupt changes with detrimental effects. For continuous monitoring in an ICU and neonatal critical care environment, EEG is one of the most practical procedures available (Jordan, 1999; Stewart et al., 2010; Abend et al., 2010; Chang and du Plessis, 2012; Riviello, 2013; Sanchez et al., 2013; Young, 2009).

Unfortunately, ICU monitoring comes paired with large quantities of data to screen, which ideally requires real-time analysis to ensure intervention if something occurs. For continuous, real-time EEG monitoring, computerized interpretation has a lot to offer. As an example, Cloostermans et al. (2011) describes a system that can be used to quantify the important neurological properties for ICU patients. Using a range of quantitative features and a decision tree based on human interpretation, EEG segments are classified into different neurological states (artifact, seizure, general periodic discharges, normal, slow wave activity, burst suppression, low-voltage, iso), and changes in states over time are presented in trends to show improvement or deterioration (Cloostermans et al., 2011).

Additionally, seizure detection algorithms, remote monitoring, and computer generated alerts can all help to improve patient treatment and clinical intervention (Abend et al., 2010; Kurtz et al., 2009; Hirsch, 2004). Remote ICU monitoring will allow neurologists to track the progress of their patients from outside the ICU, meaning changes in their neurological state can be monitored faster and on a more regular basis.

A possible next step for continuing the work in this thesis is to expand the described algorithms and add relevant features for continuous ICU monitoring. Although not all the background properties inspected here have equal value in the ICU setting, some of them (eg. symmetry and slowing), in combination additional properties (eg. burst-suppresion index, alpha-delta ratios etc.) will have high importance. The system presented in Chapter 4 can be adapted to include more relevant properties for continuous ICU monitoring, and using the same principles as implemented here to present the underlying data in a simple and intuitive manner, aid neurointensivists with better tools for bedside and even remote monitoring.

Another important contribution to ICU monitoring may involve the automated detection of seizures, in particular non-convulsive seizures which may not always be detected by ICU staff. Although the template-based spike detection method presented in Chapter 5 was designed for finding inter-ictal events, the system can easily be optimized for detecting seizures by requiring seizure events to be multiple 
successive detections of spike-wave events instead of single isolated spike-wave discharges.

\section{Non-clinical EEG and supportive technologies}

Apart from diagnostic and monitoring purposes, EEG is also growing in popularity in the consumer industry, where wireless devices are being sold with applications aimed at gaiming and assistive technologies (Duvinage et al., 2013; Khushaba et al., 2013; Webb et al., 2012; Li et al., 2013). Although many of these applications are not directly aimed at medical diagnostics or monitoring, their demand and growth will ultimately help to push EEG technology forward ${ }^{1}$.

An example of two technologies that can have a significant impact on the use of EEG, is consumer smartphones and cloud-computing (Stopczynski and Larsen, 2011; Petersen and Stahlhut, 2011; Morabito et al., 2013). The majority of people in developed countries already own a smartphone which they carry around with them throughout the day. For ambulatory monitoring, this means that an intelligent device capable of making complex calculations and sending and receiving data is already available for continuous monitoring if connected with an EEG amplifier. In special cases where the early detection of acute events can lead to an intervention by some means such as alerting a caregiver or administering a medication, the role of ambulatory monitoring will change from being passive to active. Also, smartphone technology can help to compress data during ambulatory monitoring and thereby increase the length of recordings (Morabito et al., 2013). A limitation to smartphone technology however, is that it is also battery powered and to some extent limited in computational resources, which means that complex calculations can be slower than real-time and the additional power consumption can quickly drain battery life. For this, cloud computing may bring many benefits (Ma et al., 2012). Cloud computing allows devices to upload raw data for processing and download the results when processing has been completed. A good example of this is how a smartphone can upload raw data from its GPS device to a cloud server to perform the complex calculations of obtaining a coordinate, and then download it back to the phone again when the location has been found (Ma et al., 2012). Background analysis techniques and inter-ictal spike detection can be implemented in a similar manner by letting the calculations run on the cloud. This will provide both the patient and physician with direct feedback on the recorded brain activity, without

\footnotetext{
${ }^{1} \mathrm{~A}$ good example of this can be seen at http://www.emotiv.com/.
} 
having to wait for the patient to first return to the hospital. Suitable applications for this may include monitoring a patient in real-time for ictal activity or other abnormalities, or to compress and store the data online to make longer recordings possible.

\section{Closing remarks}

This thesis has only explored a small portion of quantitative EEG analysis and automated interpretation relevant to clinical applications. Limited as they may be, most of the procedures, guidelines and algorithms described here have had positive outcomes and are of high clinical relevance and practical value. In a digital age where vast quantities of data is collected and data management becomes as important as the information it contains, automated EEG interpretation will become increasingly important to take the visual burden away from the reviewer. Automated methods will become more widely used as they improve in accuracy, and my view is that somewhere in the future, most reviewers, if not all, will make use of quantitative analysis in some form or another together with visual analysis of the raw time series data. 

Chapter 8

Nederlandse samenvatting 
In Nederland worden per jaar naar schatting veertig- tot zestigduizend routine elektro-encefalografie (EEG)-registraties verricht. Door de hoge mate van mobiliteit en de relatief lage kosten van het EEG in vergelijking met andere niet invasieve methoden voor functionele neuroimaging neemt dit aantal EEG registraties gestaag toe. Hiermee groeit echter ook de hoeveelheid te analyseren data. Conventionele methoden voor EEG analyse zijn beperkt, met name vanwege het tijdrovende karakter van visuele analyse, de training die ervoor nodig is en de kosten die ermee gepaard gaan. In dit proefschrift worden nieuwe methodes beschreven voor computer-ondersteunde EEG-interpretatie met als doel beoordelaars te assisteren in het sneller en meer consistent analyseren van EEG's door gebruik te maken van kwantitatieve en geautomatiseerde methoden. Hoewel EEG's in verschillende gebieden van diagnostiek en monitoring worden toegepast, richten we ons in dit proefschrift op de routine EEG-registraties in de poliklinische setting en de beoordeling van het EEG voor epilepsie diagnostiek. Twee gebieden van automatische analyse werden onderzocht: de analyse van het grondpatroon en de detectie van interictale epileptiforme afwijkingen. Een samenvatting van de bijdrage aan elk van deze gebieden is hieronder gegeven.

\section{Deel I: EEG grondpatroon}

Er zijn verschillende eigenschappen van het EEG grondpatroon die geanalyseerd kunnen worden, en als eerste is deze studie gestart met het ontwikkelen van een nauwkeurige techniek voor het bepalen van de frequentie van het posterieure dominante ritme (PDR). Hoewel deze frequentie in de meeste gevallen eenvoudig te meten is, is dit soms lastig door de aanwezigheid van artefacten of door een beperkt voorkomen van het PDR. Meer nauwkeurige en robuuste benaderingen werden gevonden door het EEG gedeelte waarin de ogen gesloten waren op te splitsen in kleinere segmenten en vervolgens de piekfrequenties in elk afzonderlijk segment te bepalen. Hoofdstuk 2 beschrijft een methode waarbij deze aanpak werd uitgevoerd met behulp van een curve-fitting algoritme en clustering om de piekfrequenties van de afzonderlijke segmenten te combineren. Om deze techniek te evalueren werd in een test set van 1215 EEG's de frequentie van het PDR berekend. Van al deze EEG registraties waren diagnostische beschrijvingen aanwezig, zodat berekende piekfrequentie vergeleken kon worden met de beschreven visuele beoordeling. Overeenkomsten varieerden van $96 \%$ tot $99,4 \%$ met een fouttolerantie van $1,2 \mathrm{~Hz}$. 
$\mathrm{Na}$ het vinden van een accurate techniek om de PDR frequentie robuust te meten met een volledig geautomatiseerde methode, was het volgende doel om ook de andere belangrijke eigenschappen van het EEG grondpatroon te beschrijven. Vier extra eigenschappen werden gekozen: PDR reactiviteit, links-rechts symmetrie, anterieure-posterieure gradiënten en de aan- of afwezigheid van abnormale diffuus trage activiteit. Om deze kwantitatieve interpretatie te vergelijken met de visuele beoordeling werden 384 EEG's waarin al deze vier eigenschappen gerapporteerd waren verzameld uit de database van het Medisch Spectrum Twente (MST) te Enschede. Tijdens dit proces bleek echter dat de beschrijvingen niet altijd eenduidig te interpreteren of onvolledig waren en dat er bovendien geen formele richtlijnen bestaan om deze eigenschappen te beschrijven. Daarom werd besloten om naast de ontwikkeling van een kwantitatieve methode om deze eigenschappen te beschrijven, een aantal eenvoudige maar bruikbare richtlijnen op te stellen die gebruikt kunnen worden door de beoordelaars bij het noteren van diagnostische bevindingen. Deze richtlijnen dienden tevens als definities voor de implementatie van de kwantitatieve algoritmes, zodat de automatische interpretatie ook transparant is voor de beoordelaar. Hoofdstuk drie beschrijft de gerapporteerde richtlijnen, alsmede de kwantitatieve methoden voor elke eigenschap. Deze methoden werden gevalideerd door de eigenschappen die waren verkregen met behulp van de automatische analyse te vergelijken met die van de visuele inspectie. De PDR frequentie, reactiviteit en aanwezigheid van diffuus trage activiteit hadden een matige tot hoge overeenkomst met de visuele beschrijving. Voor symmetrie en anterieureposterieure gradiënten was de overeenkomst lager. Opgemerkt dient te worden dat door de vrije tekst beschrijvingen de eigenschappen soms moeilijk in een vaste categorie voor overeenkomst te plaatsen waren.

Omdat de geautomatiseerde interpretatie methoden veelbelovende resultaten lieten zien, was het gewenst verder te testen in de klinische praktijk. Hiervoor werden deze geïmplementeerd in een zelfontworpen EEG-viewer. Samen met een nieuwe evaluatie set van 45 EEG-registraties, verkregen uit de database uit het MST in Enschede, werd deze gepresenteerd aan negen EEG-experts in Nederland voor evaluatie, zoals beschreven in Hoofdstuk 4. De EEG's varieerden genoeg om voorbeelden te bevatten van normale en afwijkende elementen van het grondpatroon. Aan de deelnemers werd gevraagd om de EEG's eerst visueel te beoordelen, en daarna de bevindingen te vergelijken met de interpretatie gemaakt door het systeem. Vervolgens werd de interbeoordelaarsbetrouwbaarheid bepaald, zowel van het geautomatiseerde systeem als van elke deelnemer, met als gouden standaard 
de consensus van alle beoordelaars. Zowel de automatische interpretatie als het merendeel van deelnemers scoorden beide hoge overeenkomst (kappa >0,6) met deze gouden standaard. In sommige gevallen scoorde automatische analyse zelfs een hogere overeenkomst dan enkele individuele deelnemers. Om de acceptatie van dergelijke algoritmes voor routine beoordelingen te bepalen, werd de deelnemers gevraagd of zij het nuttig vonden kwantitatieve analyse methoden op routine basis te gebruiken als assistentie. Hierop antwoordden alle deelnemers positief.

Uit deze studies met betrekking tot geautomatiseerde analyse van het grondpatroon kunnen we concluderen dat computer-geassisteerde interpretatie een significante bijdrage kan hebben voor routine EEG-beoordelingen. Hoewel alleen EEG's met een duur van 20-30 minuten getest zijn, zouden de algoritmen ook bruikbaar kunnen zijn in de interpretatie van langere EEG's, zoals gemaakt in ambulante registraties en voor IC-monitoring. Verder onderzoek kan helpen om de nauwkeurigheid van de interpretatie te verbeteren en om het systeem uit te breiden met aanvullende EEG eigenschappen voor beoordeling.

\section{Deel II: Interictale epileptiforme afwijkingen}

In de poliklinische setting is de diagnostisering van epilepsie een van de voornaamste redenen voor EEG-registratie. Een groot deel van dit proefschrift richt zich daarom op het vinden van een methode voor automatische detectie van interictale epileptiforme afwijkingen en deze te presenteren aan de beoordelaar, zonder dat deze eerst het gehele EEG visueel hoeft te screenen. In de literatuur is reeds een groot aantal algoritmen voor piekdetectie beschreven, echter veelal zonder klinische implementatie. Ons voornaamste doel was daarom niet alleen het vinden van nieuwe algoritmes voor het detecteren van de epileptiforme ontladingen, maar ook het creëren van een praktische oplossing die geschikt is voor klinisch gebruik.

Als bijdrage aan de automatische detectie van epileptische activiteit werden twee studies uitgevoerd. De eerste, gepresenteerd in hoofdstuk 5, beschrijft een techniek die epileptiforme ontladingen vindt door het vergelijken van alle in het EEG aanwezige signaalgolfvormen aan een getrainde database van voorbeeld-ontladingen. In dit hoofdstuk beschrijven we hoe 2160 templates van epileptiforme ontladingen zijn verkregen uit een set test EEG's. Ook beschrijven we hoe deze templates zijn getraind om vergelijkbare golfvormen te classificeren in de andere EEGs op basis van gelijkenis. Aan de templates is een maat voor betrouwbaarheid gegeven, en in combinatie met hun eigen detectie nauwkeurigheid is een stemsysteem gebruikt voor 
het groeperen van individuele gedetecteerde events. Hoewel door het bestaan van verschillende datasets geen exacte vergelijkingen gemaakt konden worden, werd in hoofdstuk 5 aangetoond dat de sensitiviteit en het aantal foutdetecties van de voorgestelde methode vergelijkbaar is met die van andere in de literatuur beschreven technieken. Bij een hogere sensitiviteit heeft deze methode meer foutdetecties door het geringe elimineren van gedekteerde events, en blijft deze in de klinische praktijk onbruikbaar in vergelijking met de standaard visuele beoordeling. Dit hoofdstuk laat echter ook zien dat de detectietechniek uitgebreid kan worden naar nieuwe, nog onbeoordeelde EEG-registraties en het daardoor grote klinische relevantie kan hebben wanneer het aantal foutdetecties wordt gereduceerd.

De tweede stap in de detectie van interictale epileptiforme activiteit was het vinden van een praktische en schaalbare oplossing voor klinisch gebruik. Dit probleem werd benaderd vanuit een ander perspectief: in plaats van een drempelwaarde te gebruiken om fout-positieven te elimineren, wijst het beschreven systeem een waarde toe voor de waarschijnlijkheid dat een gedetecteerd event inderdaad een epileptiforme afwijking is, en plaatst het deze in afnemende volgorde van waarschijnlijkheid. Vervolgens presenteert het systeem op iteratieve wijze steeds tien events aan de beoordelaar en vraagt het om een bevestiging of afwijzing voor elk getoonde event. Door dit als feedback te gebruiken leert het systeem welke events het meest van belang zijn, en past het de waarde voor de waarschijnlijkheid van de overgebleven detecties dienovereenkomstig aan. Door de beoordelaar te laten kiezen wanneer hij tevreden is met wat getoond wordt en wanneer het iteratieve proces stopt, biedt het systeem een praktische oplossing voor routine beoordelingen en kan het opgeschaald worden naar langdurige registraties. In hoofdstuk 6 werd het systeem getest op 45 routine EEG's, elk met een duur van ongeveer 20 tot 30 minuten, en lieten we zien dat na een enkele herhaling van de eerste tien getoonde events, een gemiddelde van $32 \%$ van de interictale ontladingen werd gevonden in de registraties. Na vijf herhalingen steeg dit tot $74 \%$ en na vijftien herhalingen werd 95\% van de interictale events gedetecteerd en gepresenteerd aan de beoordelaar. Dit proces kostte gemiddeld vijf minuten beoordelingstijd voor de beoordelaar per EEG.

Om de voordelen die deze techniek in de klinische praktijk kan bieden daadwerkelijk te testen is een uitgebreide evaluatie door expert elektro-encefalografisten nodig. De tot nu toe verkregen resultaten met de voorgestelde methode en beoordelingsstrategie zijn echter zeer positief. 

References 

Abend NS, Dlugos DJ, Hahn CD, Hirsch LJ, Herman ST. Use of EEG monitoring and management of non-convulsive seizures in critically ill patients: a survey of neurologists. Neurocritical care, 2010; 12:382-9.

Abend NS, Gutierrez-Colina A, Zhao H, Guo R, Marsh E, Clancy RR, et al. Interobserver reproducibility of electroencephalogram interpretation in critically ill children. J Clin Neurophysiol, 2011; 28:15-9.

Agbenu J, Newton RW, Martland T, Ismayl O, Hargreaves S. Effect of reducing the recording time of standard EEGs on the detection of EEG-abnormalities in the management of the epilepsies of childhood. Seizure, 2012; 21:422-5.

Anderson NR, Doolittle LM. Automated analysis of EEG: Opportunities and pitfalls. J Clin Neurophysiol, 2010; 27:453-7.

Anderson NR, Wisneski KJ. Automated analysis and trending of the raw EEG signal. Am J Electroneurodiagnostic Technol, 2008; 48:166-91.

Arciniegas DB. Clinical electrophysiologic assessments and mild traumatic brain injury: State-of-the-science and implications for clinical practice. Int J Psychophysiol, 2011; .

Argoud FIM, De Azevedo FM, Neto JM, Grillo E. SADE3: an effective system for automated detection of epileptiform events in long-term EEG based on context information. Med Biol Eng Comput, 2006; 44:459-70.

Askamp J, van Putten MJaM. Mobile EEG in Epilepsy. Int J Psychophysiol, 2013; .

Aurlien H, Aarseth JH, Gjerde IO, Karlsen B, Skeidsvoll H, Gilhus NE. Focal epileptiform activity described by a large computerised EEG database. Clin Neurophysiol, 2007; 118:1369-76.

Aurlien H, Gjerde IO, Aarseth JH, Eldø en G, Karlsen B, Skeidsvoll H, et al. EEG background activity described by a large computerized database. Clin Neurophysiol, 2004; 115:665-73.

Aurlien H, Gjerde IO, Gilhus NE, Hovstad OG, Karlsen B, Skeidsvoll H. A new way of building a database of EEG findings. Clin Neurophysiol, 1999; 110:986-95.

Azuma H, Hori S, Nakanishi M, Fujimoto S, Ichikawa N, Furukawa TA. An intervention to improve the interrater reliability of clinical EEG interpretations. Psychiatr Clin Neurosci, 2003; 57:485-9. 
Babiloni C, Lizio R, Carducci F, Vecchio F, Redolfi A, Marino S, et al. Resting state cortical electroencephalographic rhythms and white matter vascular lesions in subjects with Alzheimer's disease: an Italian multicenter study. J Alzheimers Dis, 2011; 26:331-46.

Babiloni C, Lizio R, Vecchio F, Frisoni GB, Pievani M, Geroldi C, et al. Reactivity of cortical alpha rhythms to eye opening in mild cognitive impairment and Alzheimer's disease: An EEG study. J Alzheim Dis, 2010; 22:1047-64.

Babiloni C, Vecchio F, Bultrini A, Luca Romani G, Rossini PM. Pre- and poststimulus alpha rhythms are related to conscious visual perception: A high-resolution EEG study. Cerebr Cortex, 2006; 16:1690-700.

Bares M, Novak T, Brunovsky M, Kopecek M, Stopkova P, Krajca V, et al. The change of QEEG prefrontal cordance as a response predictor to antidepressive intervention in bipolar depression. A pilot study. J Psychiatr Res, 2012; 46:21925.

Begić D, Popović-Knapić V, Grubišin J, Kosanović-Rajačić B, Filipčić I, Telarović I, et al. Quantitative electroencephalography in schizophrenia and depression. Psychiatr Danub, 2011; 23:355-62.

Benbadis SR, LaFrance WC, Papandonatos GD, Korabathina K, Lin K, Kraemer HC. Interrater reliability of EEG-video monitoring. Neurology, 2009; 73:843-6.

Beniczky S, Aurlien H, Brø gger JC, Fuglsang-Frederiksen A, Martins-da Silva A, Trinka E, et al. Standardized computer-based organized reporting of EEG: SCORE. Epilepsia, 2013; 54:1112-24.

Berger H. Uber das elektrenkephalogramm des menschen. Arch Psychiatr Nervenkr, 1929; 87:527-570.

Bjork M, Stovner LJ, Hagen K, Sand T. What initiates a migraine attack? Conclusions from four longitudinal studies of quantitative EEG and steady-state visualevoked potentials in migraineurs. Acta Neurol Scand Suppl, 2011; 124:56-63.

Blume WT. Drug effects on EEG. Clin Neurophysiol, 2006; 23:306-11.

Brodsky BE, Darkhovsky BS, Kaplan aY, Shishkin SL. A nonparametric method for the segmentation of the EEG. Comput Meth Programs Biomed, 1999; 60:93-106. 
Broersen P. Automatic autocorrelation and spectral analysis. Springer-Verlag New York Inc, 1 edition, 2006.

Bullmore E, Sporns O. Complex brain networks: graph theoretical analysis of structural and functional systems. Nat Rev Neurosci, 2009; 10:186-98.

Burke JF, Skolarus LE, Callaghan BC, Kerber KA. Choosing Wisely: highest-cost tests in outpatient neurology. Ann Neurol, 2013; 73:679-83.

Calzada-Reyes A, Alvarez-Amador A, Galán-García L, Valdés-Sosa M. Electroencephalographic abnormalities in antisocial personality disorder. J Forensic Leg Med, 2011; 19:29-34.

Casson AJ, Rodriguez-Villegas E. Toward online data reduction for portable electroencephalography systems in epilepsy. IEEE transactions on bio-medical engineering, 2009; 56:2816-25.

Castellanos NP, Makarov Va. Recovering EEG brain signals: artifact suppression with wavelet enhanced independent component analysis. J Neurosci Meth, 2006; 158:300-12.

Chabot RJ, Serfontein G. Quantitative electroencephalographic profiles of children with attention deficit disorder. Biol Psychiatr, 1996; 40:951-63.

Chang T, du Plessis A. Neurodiagnostic techniques in neonatal critical care. Curr Neurol Neurosci Rep, 2012; 12:145-52.

Chiang A, Rennie C, Robinson P, Roberts J, Rigozzi M, Whitehouse R, et al. Automated characterization of multiple alpha peaks in multi-site electroencephalograms. J Neurosci Methods, 2008; 168:396-411.

Chiang aKI, Rennie CJ, Robinson PA, van Albada SJ, Kerr CC. Age trends and sex differences of alpha rhythms including split alpha peaks. Clin Neurophysiol, 2011; 122:1505-17.

Cloostermans MC, de Vos CC, van Putten MJAM. A novel approach for computer assisted EEG monitoring in the adult ICU. Clin Neurophysiol, 2011; 122:2100-9.

Coburn KL, Lauterbach EC, Boutros NN, Black KJ, Arciniegas DB, Coffey CE. The value of quantitative electroencephalography in clinical psychiatry: a report by 
the Committee on Research of the American Neuropsychiatric Association. J Neuropsychiatry Clin Neurosci, 2006; 18:460-500.

Dash D, Hernandez-Ronquillo L, Moien-Afshari F, Tellez-Zenteno JF. Ambulatory EEG: a cost-effective alternative to inpatient video-EEG in adult patients. Epileptic disorders, 2012; 14:290-7.

Dauwels J, Vialatte F, Cichocki A. Diagnosis of Alzheimer's disease from EEG signals: where are we standing? Curr Alzheimer Res, 2010; 7:487-505.

De Lucia M, Fritschy J, Dayan P, Holder DS. A novel method for automated classification of epileptiform activity in the human electroencephalogram-based on independent component analysis. Med Biol Eng Comput, 2008; 46:263-72.

Delorme A, Sejnowski T, Makeig S. Enhanced detection of artifacts in EEG data using higher-order statistics and independent component analysis. Neurolmage, 2007; 34:1443-9.

Doppelbauer A, Zeitlhofer J, Zifko U, Baumgartner C, Mayr N, Deecke L. Occurrence of epileptiform activity in the routine EEG of epileptic patients. Acta Neurol Scand, 1993; 87:345-52.

Douglass LM, Wu JY, Rosman NP, Stafstrom CE. Burst Suppression Electroencephalogram Pattern in the Newborn: Predicting the Outcome. J Child Neurol, 2002; 17:403-408.

Duvinage M, Castermans T, Petieau M, Hoellinger T, Cheron G, Dutoit T. Performance of the Emotiv Epoc headset for P300-based applications. BioMedical Engineering Online, 2013; 12:56.

Ebersole JS, Pedley TA. Current practice of clinical electroencephalography. LWW medical book collection. Lippincott Williams \& Wilkins, 2003.

Faulkner HJ, Arima H, Mohamed A. Latency to first interictal epileptiform discharge in epilepsy with outpatient ambulatory EEG. Clin Neurophysiol, 2012; 123:17321735.

Fleiss J. Measuring nominal scale agreement among many raters. Psychol Bull, 1971; 76:378-382. 
Foley CM, Legido A, Miles DK, Chandler Da, Grover WD. Long-Term ComputerAssisted Outpatient Electroencephalogram Monitoring in Children and Adolescents. J Child Neurol, 2000; 15:49-55.

Foreman B, Claassen J. Annual Update in Intensive Care and Emergency Medicine 2012. Springer Berlin Heidelberg, Berlin, Heidelberg, 2012.

Friedman D, Hirsch LJ. Seizures in Critical Care. Humana Press, Totowa, NJ, 2010.

Friedman DE, Hirsch LJ. How long does it take to make an accurate diagnosis in an epilepsy monitoring unit? Clin Neurophysiol, 2009; 26:213-7.

Gaál ZA, Boha R, Stam CJ, Molnár M. Age-dependent features of EEG-reactivitySpectral, complexity, and network characteristics. Neurosci Lett, 2010; 479:79-84.

Gerber PA, Chapman KE, Chung SS, Drees C, Maganti RK, Ng YT, et al. Interobserver agreement in the interpretation of EEG patterns in critically ill adults. J Clin Neurophysiol, 2008; 25:241-9.

Gotman J, Gloor P. Automatic recognition and quantification of interictal epileptic activity in the human scalp EEG. Electroencephalogr Clin Neurophysiol, 1976; 41:513-29.

Gudmundsson S, Runarsson TP, Sigurdsson S, Eiriksdottir G, Johnsen K. Reliability of quantitative EEG features. Clin Neurophysiol, 2007; 118:2162-71.

Halford J, Shiau D, Kern R. Seizure detection software used to complement the visual screening process for long-term EEG monitoring. American Journal of Electroneurodiagnostic Technology, 2010; 133-147.

Halford JJ. Computerized epileptiform transient detection in the scalp electroencephalogram: Obstacles to progress and the example of computerized ECG interpretation. Clin Neurophysiol, 2009; 120:1909-1915.

Halford JJ, Pressly WB, Benbadis SR, Tatum WO, Turner RP, Arain A, et al. Webbased collection of expert opinion on routine scalp EEG: software development and interrater reliability. J Clin Neurophysiol, 2011; 28:178-84.

Halford JJ, Schalkoff RJ, Zhou J, Benbadis SR, Tatum WO, Turner RP, et al. Standardized Database Development for EEG Epileptiform Transient Detection: EEGnet 
Scoring System and Machine Learning Analysis. J Neurosci Meth, 2012; 212:308316.

Hammond DC. The need for individualization in neurofeedback: heterogeneity in QEEG patterns associated with diagnoses and symptoms. Appl Psychophysiol Biofeedback, 2010; 35:31-6.

Haut SR, Berg AT, Shinnar S, Cohen HW, Bazil CW, Sperling MR, et al. Interrater reliability among epilepsy centers: Multicenter study of epilepsy surgery. Epilepsia, 2002; 43:1396-401.

Herrmann CS, Arnold T, Visbeck A, Hundemer HP, Hopf HC. Adaptive frequency decomposition of EEG with subsequent expert system analysis. Comput Biol Med, 2001; 31:407-27.

Hirsch LJ. Continuous EEG monitoring in the intensive care unit: an overview. J Clin Neurophysiol, 2004; 21:332-40.

Hughes JR, John ER. Conventional and quantitative electroencephalography in psychiatry. J Neuropsychiatry, 1999; 11:190-208.

Hughes SW, Crunelli V. Thalamic mechanisms of EEG alpha rhythms and their pathological implications. Neuroscientist, 2005; 11:357-72.

Huupponen E, Himanen SL, Värri A, Hasan J, Saastamoinen A, Lehtokangas M, et al. Fuzzy detection of EEG alpha without amplitude thresholding. Artif Intell Med, 2002; 24:133-47.

Igasaki T, Higuchi T, Hayashida Y, Murayama N, Neshige R. Proposal for patientspecific automatic on-line detection of spike-and-wave discharges utilizing an artificial neural network. In 2011 4th International Conference on Biomedical Engineering and Informatics (BMEI). IEEE, 2011; 813-817.

Ille N, Berg P, Scherg M. Artifact correction of the ongoing EEG using spatial filters based on artifact and brain signal topographies. J Clin Neurophysiol, 2002; 19:113-24.

Inan ZH, Kuntalp M. A study on fuzzy C-means clustering-based systems in automatic spike detection. Comput Biol Med, 2007; 37:1160-6. 
Indiradevi KP, Elias E, Sathidevi PS, Dinesh Nayak S, Radhakrishnan K. A multilevel wavelet approach for automatic detection of epileptic spikes in the electroencephalogram. Comput Biol Med, 2008; 38:805-16.

Ishii R, Canuet L, Kurimoto R, Ikezawa K, Aoki Y, Azechi M, et al. Frontal shift of posterior alpha activity is correlated with cognitive impairment in early Alzheimer's disease: A magnetoencephalography-beamformer study. Psychogeriatrics, 2010; $10: 138-43$.

Jeong J. EEG dynamics in patients with Alzheimer's disease. Clin Neurophysiol, 2004; 115:1490-505.

Ji Z, Sugi T, Goto S, Wang X, Ikeda A, Nagamine T, et al. An automatic spike detection system based on elimination of false positives using the large-area context in the scalp EEG. IEEE Trans Biomed Eng, 2011a; 58:2478-88.

Ji Z, Wang X, Sugi T, Goto S, Nakamura M. Automatic spike detection based on realtime multi-channel template. 2011 4th International Conference on Biomedical Engineering and Informatics (BMEI), 2011b; 648-652.

Jiang L, Yin X, Yin C, Zhou S, Dan W, Sun X. Different quantitative EEG alterations induced by TBI among patients with different APOE genotypes. Neurosci Lett, 2011; 505:160-4.

Jin Y, Potkin SG, Kemp AS, Huerta ST, Alva G, Thai TM, et al. Therapeutic effects of individualized alpha frequency transcranial magnetic stimulation (alphaTMS) on the negative symptoms of schizophrenia. Schizophr Bull, 2006; 32:556-61.

John ER, Prichep LS, Alper KR, Mas FG, Cancro R, Easton P, et al. Quantitative electrophysiological characteristics and subtyping of schizophrenia. Biol Psychiatr, 1994; 36:801-26.

Jordan K. Continuous EEG monitoring in the neuroscience intensive care unit and emergency department. J Clin Neurophysiol, 1999; .

Jordan KG. Neurophysiologic monitoring in the neuroscience intensive care unit. Neurologic clinics, 1995; 13:579-626.

Kannathal N, Choo ML, Acharya UR, Sadasivan PK. Entropies for detection of epilepsy in EEG. Comput Methods Programs Biomed, 2005; 80:187-94. 
Kemp B, Blom H. Optimal detection of the alpha state in a model of the human electroencephalogram. Electroencephalogr Clin Neurophysiol, 1981; 52:222-225.

Khushaba RN, Wise C, Kodagoda S, Louviere J, Kahn BE, Townsend C. Consumer neuroscience: Assessing the brain response to marketing stimuli using electroencephalogram (EEG) and eye tracking. Expert Syst Appl, 2013; 40:3803-3812.

Klass D. The continuing challenge of artifacts in the EEG. Am J EEG Technol, 1995;

Klimesch W. EEG-alpha rhythms and memory processes. Int J Psychophysiol, 1997; 26:319-40.

Knyazeva MG, Jalili M, Meuli R, Hasler M, De Feo O, Do KQ. Alpha rhythm and hypofrontality in schizophrenia. Acta Psychiatr Scand, 2008; 118:188-99.

Könönen M, Partanen JV. Blocking of EEG alpha activity during visual performance in healthy adults. A quantitative study. Electroencephalogr Clin Neurophysiol, 1993; 87:164-6.

Korotchikova I, Stevenson NJ, Walsh BH, Murray DM, Boylan GB. Quantitative EEG analysis in neonatal hypoxic ischaemic encephalopathy. Clin Neurophysiol, 2011; .

Kumar Y, Dewal ML, Anand RS. Epileptic seizures detection in EEG using DWTbased ApEn and artificial neural network. Res J Appl Sci Eng Tech, 2012; 4:35883591.

Kurtz P, Hanafy KA, Claassen J. Continuous EEG monitoring: Is it ready for prime time? Curr Opin Crit Care, 2009; 15:99-109.

Lachaux JP, Rodriguez E, Martinerie J, Varela FJ. Measuring phase synchrony in brain signals. Hum Brain Mapp, 1999; 8:194-208.

Landis JR, Koch GG. The measurement of observer agreement for categorical data. Biometrics, 1977; 33:159-74.

Leach JP, Stephen LJ, Salveta C, Brodie MJ. Which electroencephalography (EEG) for epilepsy? The relative usefulness of different EEG protocols in patients with possible epilepsy. J Neurol Neurosurg Psychiatry, 2006; 77:1040-2. 
Lee SH, Park YM, Kim DW, Im CH. Global synchronization index as a biological correlate of cognitive decline in Alzheimer's disease. Neurosci Res, 2010; 66:3339.

Levenberg K. A Method for the Solution of Certain Non-Linear Problems in Least Squares. Q Appl Math, 1944; 2:164-168.

Levin KH, Lüders H. Comprehensive clinical neurophysiology. W.B. Saunders, 2000.

Li M, Zhang Y, Zhang H, Hu HS. An EEG Based Control System for Intelligent Wheelchair. Appl Mech Mater, 2013; 300-301:1540-1545.

Lodder SS, Askamp J, van Putten MJAM. Inter-ictal spike detection using a database of smart templates. Clin Neurophysiol, 2013; .

Lodder SS, van Putten MJAM. Automated EEG analysis: Characterizing the posterior dominant rhythm. J Neurosci Meth, 2011; 200:86-93.

Lodder SS, van Putten MJAM. Quantification of the adult EEG background pattern. Clin Neurophysiol, 2013; 124:228-37.

Logi F, Pasqualetti P, Tomaiuolo F. Predict recovery of consciousness in post-acute severe brain injury: The role of EEG reactivity. Brain Inj, 2011; 25:972-979.

Lopes da Silva FH, Pijn JP, Velis D, Nijssen PC. Alpha rhythms: noise, dynamics and models. Int J Psychophysiol, 1997; 26:237-49.

Ma X, Cui Y, Stojmenovic I. Energy Efficiency on Location Based Applications in Mobile Cloud Computing: A Survey. Procedia Computer Science, 2012; 10:577584 .

Marcuse LV, Schneider M, Mortati KA, Donnelly KM, Arnedo V, Grant AC. Quantitative analysis of the EEG posterior-dominant rhythm in healthy adolescents. Clin Neurophysiol, 2008; 119:1778-81.

Martin W, Johnson L, Viglione S, Naitoh P, Joseph R, Moses J. Pattern recognition of EEG-EOG as a technique for all-night sleep stage scoring. Electroencephalogr Clin Neurophysiol, 1972; 32:417-427.

Maulsby RL, Kellaway P, Graham M. The normative electroencephalographic data reference library. Final report, contract NAS-9-1200 Washington DC National aeronautics and space administration, 1968; . 
Mishra M, Banday M, Derakhshani R, Croom J, Camarata PJ. A quantitative EEG method for detecting post clamp changes during carotid endarterectomy. J Clin Monit Comput, 2011; 25:295-308.

Moeller JJ, Tu B, Bazil CW. Quantitative and qualitative analysis of ambulatory electroencephalography during mild traumatic brain injury. Arch Neurol, 2011; 68:1595-8.

Morabito FC, Labate D, Morabito G, Palamara I, Szu H. Monitoring and diagnosis of Alzheimer's disease using noninvasive compressive sensing EEG. volume 8750. 2013; 87500Y-87500Y-10.

Moretti DV, Zanetti O, Binetti G, Frisoni GB. Quantitative EEG Markers in Mild Cognitive Impairment: Degenerative versus Vascular Brain Impairment. Int J Alzheimers Dis, 2012; 2012:917537.

Mukhopadhyay S, Ray GC. A new interpretation of nonlinear energy operator and its efficacy in spike detection. IEEE Trans Biomed Eng, 1998; 45:180-7.

Nakamura M, Shibasaki H, Imajoh K, Nishida S, Neshige R, Ikeda A. Automatic EEG interpretation: a new computer-assisted system for the automatic integrative interpretation of awake background EEG. Electroencephalogr Clin Neurophysiol, 1992; 82:423-431.

Nakamura M, Sugi T, Ikeda A, Shibasaki H. Automatic EEG interpretation adaptable to individual electroencephalographer using artificial neural network. Int J Adapt Contr Signal Process, 2002; 16:25-37.

Naruse Y, Matani A, Miyawaki Y, Okada M. Influence of coherence between multiple cortical columns on alpha rhythm: a computational modeling study. Human brain mapping, 2010; 31:703-15.

Nenadic Z, Burdick JW. Spike detection using the continuous wavelet transform. IEEE Trans Biomed Eng, 2005; 52:74-87.

Niedermeyer E, da Silva FL. Electroencephalography: Basic Principles, Clinical Applications, and Related Fields. Lippincott Williams \& Wilkins, 5th edition, 2004.

Nonclercq A, Foulon M, Verheulpen D, De Cock C, Buzatu M, Mathys P, et al. Spike detection algorithm automatically adapted to individual patients applied 
to spike-and-wave percentage quantification. Neurophysiol Clin Clin Neurophysiol, 2009; 39:123-31.

Nonclercq A, Foulon M, Verheulpen D, De Cock C, Buzatu M, Mathys P, et al. Cluster-based spike detection algorithm adapts to interpatient and intrapatient variation in spike morphology. J Neurosci Methods, 2012; 210:259-265.

Nunez PL, Ramesh S. Electric Fields of the Brain: The Neurophysics of EEG, 2nd Edition. Oxford University Press, USA, 2005.

Nuwer M. Assessment of digital EEG, quantitative EEG, and EEG brain mapping: Report of the American Academy of Neurology and the American Clinical Neurophysiology Society. Neurology, 1997; 49:277-292.

Obrist WD. Problems of aging. In Remond A (ed). Handbook of electroencephalography and Clinical Neurophysiology. Elsevier Amsterdam, 1976.

Orekhova EV, Elam M, Orekhov VY. Unraveling superimposed EEG rhythms with multi-dimensional decomposition. J Neurosci Methods, 2011; 195:47-60.

Partanen J, Soininen H, Helkala E, Könönen M, Kilpeläinen R, Riekkinen P. Relationship between EEG reactivity and neuropsychological tests in vascular dementia. J Neural Transm, 1997; 104:905-912.

Petersen M, Stahlhut C. Smartphones get emotional: mind reading images and reconstructing the neural sources. Affective Computing and Intelligent Interaction, $2011 ; 578-587$.

Petit D, Gagnon JF, Fantini ML, Ferini-Strambi L, Montplaisir J. Sleep and quantitative EEG in neurodegenerative disorders. J Psychosom Res, 2004; 56:487-96.

Prichep LS, John ER, Ferris SH, Reisberg B, Almas M, Alper K, et al. Quantitative EEG correlates of cognitive deterioration in the elderly. Neurobiol Aging, 1994; 15:85-90.

Ramachandrannair R, Sharma R, Weiss SK, Cortez MA. Reactive EEG patterns in pediatric coma. Pediatr Neurol, 2005; 33:345-9.

Riviello JJ. Digital trend analysis in the pediatric and neonatal intensive care units. J Clin Neurophysiol, 2013; 30:143-55. 
Robinson P, Loxley P, O'Connor S, Rennie C. Modal analysis of corticothalamic dynamics, electroencephalographic spectra, and evoked potentials. Phys Rev E, 2001; 63:1-13.

Robinson P, Whitehouse R, Rennie C. Nonuniform corticothalamic continuum model of electroencephalographic spectra with application to split-alpha peaks. Physical Review E, 2003; 68:1-10.

San-juan D, Chiappa KH, Cole AJ. Propofol and the electroencephalogram. Clin Neurophysiol, 2010; 121:998-1006.

Sanchez SM, Carpenter J, Chapman KE, Dlugos DJ, Gallentine WB, Giza CC, et al. Pediatric ICU EEG monitoring: current resources and practice in the United States and Canada. J Clin Neurophysiol, 2013; 30:156-60.

Scherg M, Ille N, Weckesser D, Ebert A, Ostendorf A, Boppel T, et al. Fast evaluation of interictal spikes in long-term EEG by hyper-clustering. Epilepsia, 2012; 53:1196-204.

Schomer DL, Lopes da Silva FH. Niedermeyer's Electroencephalography: Basic Principles, Clinical Applications, and Related Fields. Lippincott Williams \& Wilkins, 2010.

Segalowitz SJ, Santesso DL, Jetha MK. Electrophysiological changes during adolescence: A review. Brain Cognit, 2010; 72:86-100.

Segrave RA, Thomson RH, Cooper NR, Croft RJ, Sheppard DM, Fitzgerald PB. Upper alpha activity during working memory processing reflects abnormal inhibition in major depression. J Affect Disord, 2010; 127:191-8.

Seneviratne U, Mohamed A, Cook M, D'Souza W. The utility of ambulatory electroencephalography in routine clinical practice: a critical review. Epilepsy research, 2013; 105:1-12.

Sewards TV, Sewards MA. Alpha-band oscillations in visual cortex: Part of the neural correlate of visual awareness? Int J Psychophysiol, 1999; 32:35-45.

Shen CP, Liu ST, Zhou WZ, Lin FS, Lam AYY, Sung HY, et al. A physiology-based seizure detection system for multichannel EEG. PloS one, 2013; 8:e65862. 
Sigl JC, Chamoun NG. An introduction to bispectral analysis for the electroencephalogram. J Clin Monit, 1994; 10:392-404.

Silva LD. Neural mechanisms underlying brain waves: from neural membranes to networks. Electroencephalogr Clin Neurophysiol, 1991; 79:81-93.

Snaedal J, Johannesson GH, Gudmundsson TE, Blin NP, Emilsdottir AL, Einarsson B, et al. Diagnostic accuracy of statistical pattern recognition of electroencephalogram registration in evaluation of cognitive impairment and dementia. Dement Geriatr Cogn Disord, 2012; 34:51-60.

Spronk D, Arns M, Barnett KJ, Cooper NJ, Gordon E. An investigation of EEG, genetic and cognitive markers of treatment response to antidepressant medication in patients with major depressive disorder: A pilot study. J Affect Disord, 2011; 128:41-8.

Stam CJ. Nonlinear dynamical analysis of EEG and MEG: review of an emerging field. Clin Neurophysiol, 2005; 116:2266-301.

Stam CJ, Jones BF, Nolte G, Breakspear M, Scheltens P. Small-world networks and functional connectivity in Alzheimer's disease. Cereb Cortex, 2007; 17:92-9.

Stam CJ, Pijn JP, Suffczynski P, Lopes da Silva FH. Dynamics of the human alpha rhythm: evidence for non-linearity? Clin Neurophysiol, 1999; 110:1801-13.

Steriade M, Gloor P, Llinas R. Basic mechanisms of cerebral rhythmic activities. Electroencephalogr, 1990; 76:481-508.

Stevens A, Kircher T. Cognitive decline unlike normal aging is associated with alterations of EEG temporo-spatial characteristics. Eur Arch Psychiatr Clin Neurosci, 1998; 248:259-66.

Stevenson NJ, Korotchikova I, Temko A, Lightbody G, Marnane WP, Boylan GB. An automated system for grading EEG abnormality in term neonates with hypoxicischaemic encephalopathy. Ann Biomed Eng, 2013; 41:775-85.

Stewart CP, Otsubo H, Ochi a, Sharma R, Hutchison JS, Hahn CD. Seizure identification in the ICU using quantitative EEG displays. Neurology, 2010; 75:1501-8.

Stopczynski A, Larsen J. A smartphone interface for a wireless EEG headset with real-time $3 \mathrm{D}$ reconstruction. Affective Computing and Intelligent Interaction, $2011 ; 4: 317-318$. 
Strzelczyk A, Nickolay T, Bauer S, Haag A, Knake S, Oertel WH, et al. Evaluation of health-care utilization among adult patients with epilepsy in Germany. Epilepsy Behav, 2012; 23:451-7.

Subasi A. Automatic detection of epileptic seizure using dynamic fuzzy neural networks. Expert Syst Appl, 2006; 31:320-328.

Tatum WO, Dworetzky Ba, Freeman WD, Schomer DL. Artifact: recording EEG in special care units. J Clin Neurophysiol, 2011a; 28:264-77.

Tatum WO, Dworetzky Ba, Schomer DL. Artifact and recording concepts in EEG. J Clin Neurophysiol, 2011b; 28:252-63.

Thakor NV, Tong S. Advances in quantitative electroencephalogram analysis methods. Annu Rev Biomed Eng, 2004; 6:453-95.

Thatcher RW. Validity and Reliability of Quantitative Electroencephalography. Journal of Neurotherapy, 2010; 14:122-152.

Trevathan E. Ellen R. Grass Lecture: Rapid EEG analysis for intensive care decisions in status epilepticus. Am J Electroneurodiagnostic Technol, 2006; 46:4-17.

van de Velde M, Ghosh IR, Cluitmans PJ. Context related artefact detection in prolonged EEG recordings. Comput Meth Programs Biomed, 1999; 60:183-96.

van der Hiele K, Vein AA, Reijntjes RHAM, Westendorp RGJ, Bollen ELEM, van Buchem MA, et al. EEG correlates in the spectrum of cognitive decline. Clin Neurophysiol, 2007; 118:1931-9.

van der Stelt O. Development of human EEG posterior alpha rhythms. Clin Neurophysiol, 2008; 119:1701-2.

Van Hese P, Vanrumste B, Hallez H, Carroll GJ, Vonck K, Jones RD, et al. Detection of focal epileptiform events in the EEG by spatio-temporal dipole clustering. Clin Neurophysiol, 2008; 119:1756-70.

van Putten MJAM. Nearest neighbor phase synchronization as a measure to detect seizure activity from scalp EEG recordings. J Clin Neurophysiol, 2003; 20:320-5.

van Putten MJAM. The colorful brain: Visualization of EEG background patterns. J Clin Neurophysiol, 2008; 25:63-8. 
van Putten MJAM. Essentials of Neurophysiology: Basic Concepts and Clinical Applications for Scientists and Engineers. 230. Springer, 2009;

van Putten MJAM, Kind T, Visser F, Lagerburg V. Detecting temporal lobe seizures from scalp EEG recordings: A comparison of various features. Clin Neurophysiol, $2005 ; 116: 2480-9$.

van Putten MJAM, Peters JM, Mulder SM, de Haas JAM, Bruijninckx CMA, Tavy DLJ. A brain symmetry index (BSI) for online EEG monitoring in carotid endarterectomy. Clin Neurophysiol, 2004; 115:1189-94.

van Putten MJAM, Tavy DLJ. Continuous quantitative EEG monitoring in hemispheric stroke patients using the brain symmetry index. Stroke, 2004; 35:2489-92.

van Vugt MK, Sederberg PB, Kahana MJ. Comparison of spectral analysis methods for characterizing brain oscillations. J Neurosci Methods, 2007; 162:49-63.

Vigário R, Särelä J, Jousmäki V, Hämäläinen M, Oja E. Independent component approach to the analysis of EEG and MEG recordings. IEEE transactions on biomedical engineering, 2000; 47:589-93.

Wang C, Zou J, Zhang J, Wang M, Wang R. Feature extraction and recognition of epileptiform activity in EEG by combining PCA with ApEn. Cogn Neurodyn, 2010; 4:233-40.

Waterhouse E. New horizons in ambulatory electroencephalography. IEEE Eng Med Biol Mag, 2003; 22:74-80.

Webb J, Xiao ZG, Aschenbrenner KP, Herrnstadt G, Menon C. Towards a portable assistive arm exoskeleton for stroke patient rehabilitation controlled through a brain computer interface. In 2012 4th IEEE RAS \& EMBS International Conference on Biomedical Robotics and Biomechatronics (BioRob). IEEE, 2012; 12991304.

Willems JL, Arnaud P, van Bemmel JH, Bourdillon PJ, Brohet C, Dalla Volta S, et al. Assessment of the performance of electrocardiographic computer programs with the use of a reference data base. Circulation, 1985; 71:523-534.

Wilson SB, Emerson R. Spike detection: A review and comparison of algorithms. Clin Neurophysiol, 2002; 113:1873-81. 
Young GB. Continuous EEG monitoring in the ICU: challenges and opportunities. Can J Neurol Sci, 2009; 36 Suppl 2:S89-91.

Zhang X, Wang X, Sugi T, Ikeda A, Nagamine T, Shibasaki H, et al. Automatic interpretation of hyperventilation-induced electroencephalogram constructed in the way of qualified electroencephalographer's visual inspection. Med Biol Eng Comput, 2011; 49:171-80.

Zhou J, Schalkoff RJ, Dean BC, Halford JJ. Morphology-based wavelet features and multiple mother wavelet strategy for spike classification in EEG signals. Conference proceedings : Annual International Conference of the IEEE Engineering in Medicine and Biology Society, 2012; 2012:3959-62. 


\section{ACKNOWLEDGMENTS}

It has been an amazing four years in which I have learned a lot about a field that I could only imagine to be familiar with a few years ago. The ride has been fun, but as with all good things, it has to come to an end to make space for the next adventure. Many people have played a key role for me to be where I am today, both directly and indirectly. I would like to thank all of them: friends, family, colleagues, relationships and acquaintances. Without your support and encouragement this thesis would not have been possible.

I would also like to thank a number of people in particular. First, my parents for their continuous support and encouragement over the course of this project. Your weekly phone calls left me recharged and ready for the week ahead, and I am grateful for the opportunity you gave me to study and for believing in me. To my brothers Johnathan and Jason, thank you for your support. Seeing both of you so passionate in what you do is a great inspiration to me. Never stop dreaming!

My next thank you goes to Michel van Putten for allowing me to make this PhD a reality. Without his exchange of ideas and concepts this project would not have reached its full potential. I still remember that first phone interview on an unexpected evening in South Africa. The signal quality was poor (or maybe surfing just left my ears full of sand and water), but regardless of barely hearing anything, I was determined to leave a good impression. Luckily with Michel this proved to be easy given his natural style of making one feel comfortable and equal in a conversation. Michel, thank you for taking a chance with me and allowing me to pursue this project in a style and manner that fits my personality. I appreciate the freedom you gave me and particularly value the working environment that you put together where a simple engineer can learn and eventually contribute to a field as different as clinical neurophysiology. Our meetings and discussions were greatly valued, and I hope that more discussions still lie ahead. 
Over the course of this project I have often relied on colleagues and fellow PhD students to help me with translations, proofreading, feedback on design protocols and many administrative tasks which I was happy to delegate to whomever I could find. Apart from this, I was also invited into the personal lives of many colleagues which made me feel at home in a foreign country and made the working environment very pleasurable. To Bas-Jan, Cecile, Chin, Esther, Floor, Ghita, Hanneke, Jessica, Marleen and Sid, thank you. I wish all of you the best for your future careers, and maybe one day our paths will cross again. To Jeannette, sharing an office with you has been a real pleasure. After that day when you brought a massive box of coffee to work I knew that we will get along just fine. To the rest of the ECTM staff and researchers that I spent lunch, meetings, and coffee breaks with, thanks for all the laughs and good debates that we had. Special thanks also to Danielle, Esmeralda, Jolanda and Tanja for helping me with all the administrative tasks. Also, thank you to Linda, Marleen and Esther for helping me with the Dutch translations of this thesis.

At the start of this project I only had a small amount of experience with electroencephalography, and although this still remains limited, I learned a lot during the early months with the help of the technical staff at the Clinical Neurophysiology department at Medisch Spectrum Twente in Enschede. By allowing me to observe and make notes during patient measurements, I was able to quickly become familiar with the field, and together with Thursday morning meetings in the hospital led by Michel, my informal training into EEGs has given me an invaluable tool that I can carry forward with me. Thank you in particular to Carin and Tom for making my days in the hospital more fun. We all know how much I like hospitals..

I had the fortunate opportunity to also supervise three bachelors and Masters students during the course of my PhD. To Astrid, Michiel and Sanne, thank you for all the ideas and insights that we shared. It was a pleasure working with you. To all the other students I had the opportunity to work with, especially those that provided me with requests and suggestions to make my software and algorithms better, thank you very much.

To all my friends and family abroad, Alex, Almero, Andrea, Ben, Bevan, Bjorn, Ciske, Colleen, Fauve, Gerhard, Henry, Jar, Jaques, Juergen, Neil, Ockie, Petrus, Rayno, Ross, Thomas, Tjoppie and van Wyk, you have played a large role in this project. Your Skype calls and emails were the highlight of my day and made me feel much closer to home. As anyone who lives abroad knows, there are days when it all seems too much. Thank you for motivating me to keep going. 
To everyone that I have met during my stay in the Netherlands, thank you for making me feel at home. Having so many great friends to relax with has surely made this project easier. Thanks in particular to Aard, Anne, Bart, Bram, Brian, David, Erwin, Evelien, Feite, Frank, Frank, Freek, Gregory, Japie, Kazia, Linda, Marcel, Merel, Natasja, Nick, Niels, Niels, Peter, Precivil, Ramazan, Roeland, Samuele, Sanne, Stijn, Taco, Tjeerd and Yusuf and all the other people I've met. Although I miss many names here, the other 200 of you are just as awesome! From burger groups to surf trips and escalating Friday nights, I had a lot of fun!

Lastly I would like to thank all the people that participated in one way or another to this research. Apart from Michel and fellow researchers, I was also fortunate enough to have a group of very busy and very patient neurologists and clinical neurophysiologists to help me with evaluation and testing: Dr. Geert Brekelmans, Prof. Oebele F. Brouwer, Prof. J. Gert van Dijk, Dr. Jeannette Hofmeijer, Dr. Frans S. S. Leijten, Dr. Jan Meulstee, Dr. Jaco Pasman, Dr. Robjan Schimsheimer, and Dr. Selma C. Tromp. Thank you all. Your participation and feedback is greatly valued and I hope that you had as much pleasure working with me as I did in working on the methods and algorithms that may one day help you.

Lastly, I would like to thank my PhD defence committee for reading my thesis and for taking part in my PhD defence. Also thank you for the wonderful and very insightful questions that you have asked.

May the next adventure be as fun as this one.

Shaun 



\section{About THE AUthOR}

Shaun Sandy Lodder was born on 20 October 1984 in Kimberley, South Africa. He graduated high school in 2002, and after spending a year abroad in the United Kingdom, he returned to South Africa and studied engineering at the University of Stellenbosch. His main areas of interest included applied mathematics, software design, computer vision and machine learning. In 2008 he obtained his bachelor's degree and continued to complete a master's degree in engineering in 2010 under supervision of prof. Johan A. du Preez. The topic of his master's thesis was brain-computer interfaces. After finishing his master's degree, Shaun moved to the Netherlands to continue his research as a doctoral student in automated EEG analysis under supervision of prof. Michel J.A.M van Putten. He obtained his PhD in 2014 and moved back to South Africa, where he founded a company to bring his research to clinical practice. In his spare time, Shaun enjoys being outdoors as much as possible and in particular loves surfing, kitesurfing, wine-tasting, diving, and a variety of other adrenaline-filled activities. 